\title{
Units of Weight and Measure
}

(United States Customary and Metric)

\section{Definitions and Tables of Equivalents}

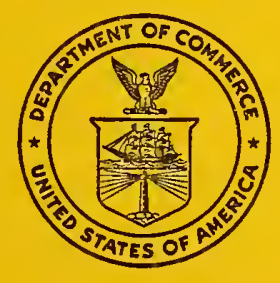

United States Department of Commerce

National Bureau of Standards

Miscellaneous Publication 214 


\section{PERIODIGALS OF THE NATIONAL BUREAU OF STANDARDS}

\section{(Published monthly)}

The National Bureau of Standards is engaged in fundamental and applied research in physics, chemistry, mathematics, and engineering. Projects are conducted in fifteen fields: electricity and electronics, optics and metrology, heat and power, atomic and radiation physics, chemistry, mechanics, organic and fibrous materials, metallurgy, mineral products, building technology, applied mathematics, data processing systems, cryogenic engineering, radio propagation, and radio standards. The Bureau has custody of the national standards of measurement and conducts research leading to the improvement of scientific and engineering standards and of techniques and methods of measurement. Testing methods and instruments are developed; physical constants and properties of materials are determined; and technical processes are investigated.

\section{Journal of Research}

The Journal presents research papers by authorities in the specialized fields of physics, mathematics, chemistry, and engineering. Complete details of the work are presented, including laboratory data, experimental procedures, and theoretical and mathematical analyses. Annual subscription: domestic, $\$ 4.00$; foreign, $\$ 5.25$.

\section{Technical News Bulletin}

Summaries of current research at the National Bureau of Standards are published each month in the Technical News Bulletin. The articles are brief, with emphasis on the results of research, chosen on the basis of their scientific or technologic importance. Lists of all Bureau publications during the preceding month are given, including Research Papers, Handbooks, Applied Mathematics Series, Building Materials and Structures Reports, Miscellaneous Publications, and Circulars. Each issue contains 12 or more two-column pages; illustrated. Annual subscription: domestic, $\$ 1.00$; foreign, $\$ 1.35$.

\section{Basic Radio Propagation Predictions}

The Predictions provide the information necessary for calculating the best frequencies for communication between any two points in the world at any time during the given month. The data are important to all users of long-range radio communications and navigation, including broadcasting, airline, steamship, and wireless services, as well as to investigators of radio propagation and ionosphere. Each issue, covering a period of one month, is released three months in advance and contains 16 large pages, including pertinent charts, drawings, and tables. Annual subscription: domestic, $\$ 1.00$; foreign, $\$ 1.25$.

\section{GATALOG OF NBS PUBLICATIONS}

National Bureau of Standards Circular 460 and its Supplement list all Bureau publications from 1901 through June 1952, including Applied Mathematics Series, Building Materials and Structures Reports, Circulars, Handbooks, Research Papers, and Miscellaneous Publications. Brief abstracts for the publications issued after January 1, 1942, are also included.

National Bureau of Standards Circular 460, 375 pages, \$1.25. Supplement to Circular 460, 223 pages, 75 cents. (A free mimeographed list of publications issued since June 1952 is available on request to the National Bureau of Standards). 


\section{Units of Weight and Measure}

(United States Customary and Metric)

\section{Definitions and Tables of Equivalents}

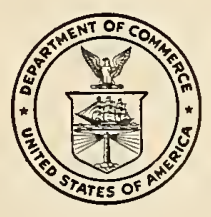

National Bureau of Standards Miscellaneous Publication 214 Issued July 1, 1955

(Supersedes Miscellaneous Publication 121) 



\section{CONTENTS}

2. Fundamental equivalents

3.1. Length

3.2. Area

3.3. Volume

3.4. Capacity

3.5. Mass.........

4. Standards of measurement

5. Spelling and abbreviation of units.

5.1. List of the most common units of weight and measure and their abbreviations _- 7

6. Tables of interrelation of units of measurement

6.1. Length

6.2. Area

6.3. Volume

6.4. Capacity-Liquid measure

6.5. Capacity-Dry measure

6.6. Mass-Not greater than pounds and kilograms

6.7. Mass-Not less than avoirdupois ounces

7. Comparison of metric and customary units from 1 to 9

7.1. Length

7.2. Area

7.3. Volume

7.4. Capacity-Liquid measure

7.5. Capacity-Dry measure

7.6. Mass

7.7. Comparison of the various tons and pounds in use in the United States (from 1 to 9 units)

8. Special tables.....

Length:

Inches and millimeters-equivalents of decimal and binary fractions of an inch, in millimeters, from $1 / 64$ to 1 inch

Hundredths of an inch to millimeters, from 1 to 99 hundredths

Millimeters to decimals of an inch, from 1 to 99 units

United States nautical miles, international nautical miles, and kilometers...-

Millimeters to inches.

9. Tables of equivalents from 1 to 999 units

Length:

Feet to meters

Meters to feet.

Miles to kilometers

Kilometers to miles_............ 34

Area:

Acres to hectares

Volume:

Hectares to acres.-. 38

Cubic yards to cubic meters

Cubic meters to cubic yards 
9. Tables of equivalents from 1 to 999 units-Continued

Capacity:

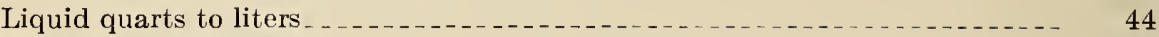

Liters to liquid quarts

Gallons to liters

Liters to gallons............... 50

Bushels to hectoliters Mass:

Hectoliters to bushels_._.

Avoirdupois pounds to kilograms_...

Kilograms to avoirdupois pounds

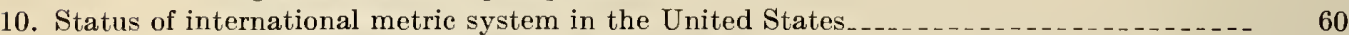

10.1. Constitutional authority to fix standards

10.2. Metric system legalized_._.

10.3. Metric standards adopted as fundamental

10.4. Metric balances furnished certain post offices

10.5. Metric weights used for certain coins

10.6. Congress provides metric standards for the States of the Union, land-grant agricultural colleges, and the Smithsonian Institution_........................

10.7. United States joins in establishing International Bureau of Weights and

Measures_..................

10.8. Congress adopts the metric units for electrical measures____

10.9. Metric carat adopted as unit of weight for diamonds and other precious stones

10.10. Metric system optional in Puerto Rico

10.11. Relation between inches and millimeters adopted by American Standards Association 


\title{
Units of Weight and Measure
}

\section{(United States Customary and Metric)}

\section{Definitions and Tables of Equivalents}

\author{
L. V. Judson
}

\begin{abstract}
The units of length, area, volume, capacity, and mass in use in the United States are defined. Tables of interrelation and tables of equivalents for these units in the metric system and in the U. S. customary system are given. Such tables are needed in scientific, industrial, commercial, and other fields, and have been published by the United States Government for many years.

There is a section in which the fundamental equivalents are stated, one containing the approved spelling and abbreviation of each of the most common units of weight and measure, and one showing the status of the metric system in the United States. All of the tables and other material have been revised to conform to the definitions, equivalents, and usages current at the date of issue. The conversion tables are confined to simple units, excluding all compound units such as foot-pounds, pounds per cubic foot, and feet per second.
\end{abstract}

\section{INTRODUCTION}

This publication supersedes National Bureau of Standards Miscellaneous Publication 121, issued in January 1936. Necessary corrections have been made, and the text has been revised and rearranged. Tabular values involving the liter have been changed to the basis 1 Jiter $=1.000028$ cubic decimeters; the international nautical mile of 1,852 meters, as adopted by the Departments of Defense and Commerce on July 1, 1954, has been substituted for the older U. S. nautical mile; and the information on the status of the metric system in the United States has been brought up to date.

In the construction of the tables in this publication, when the fundamental relation of the units furnished directly a reduction factor for use in determining the multiples of the units, this factor was used in its fundamental form, as, for example, that 1 meter= 39.37 inches. Reduction factors which it was necessary to obtain, however, by multiplication, division, powers, or roots, etc., of the fundamental relations were usually carried out to a greater degree of accuracy than that to which it is usually possible to make measurements, for convenience in computing the multiples to the accuracy desired.

When the tables do not give the equivalent of any desired quantity directly and completely, the equivalent can usually be obtained, without the necessity of making a multiplication of these reduction factors, by using quantities from several tables, making a shift of decimal points, if necessary, and merely adding the results. 
The supplementary metric units are formed by combining the words "meter", "gram", and "liter" with the six numerical prefixes, as in the following table:

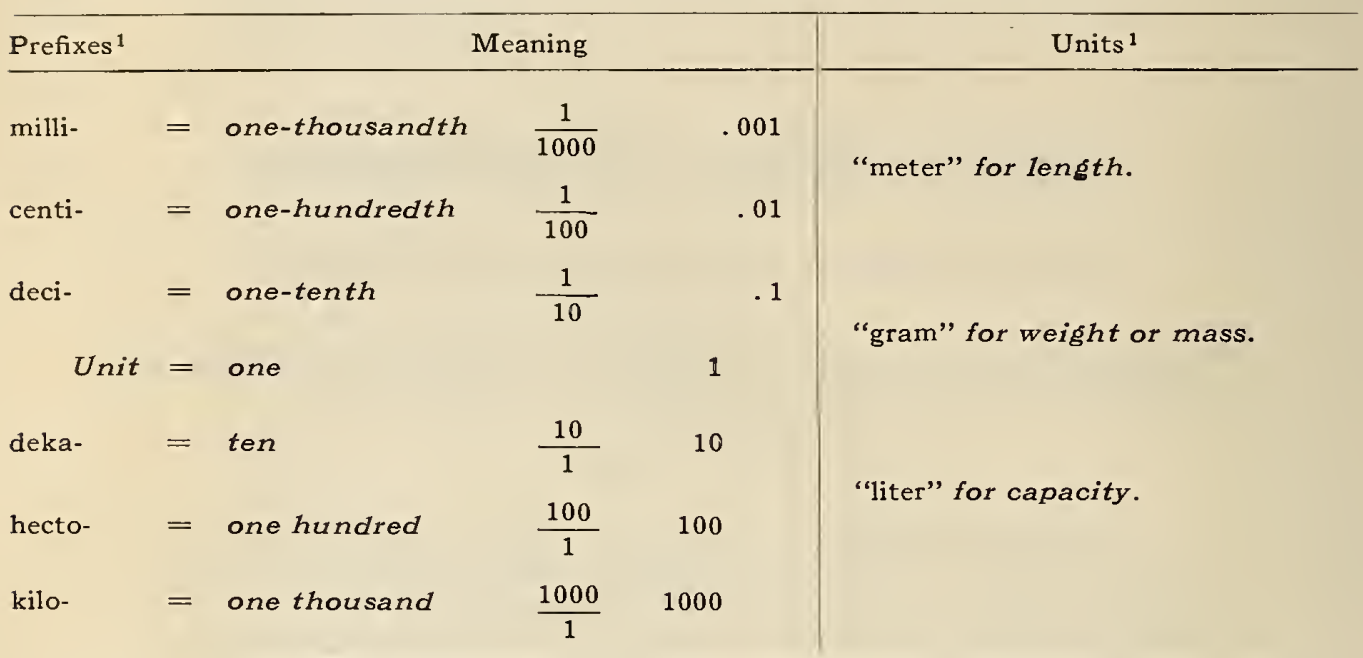

Not all of the 18 possible combinations of the above prefixes and units are in common use; for example the dekameter and the hectometer are seldom found except in text books.

All lengths, areas, and cubic measures in the following tables are derived from the international meter, the basic relation between units of the United States customary and the metric systems being:

$$
1 \text { meter }=39.37 \text { inches, }
$$

contained in the law of July 28, 1866, ${ }^{1}$ and set forth in the Mendenhall Order ${ }^{2}$ of April 5, 1893, namely:

$$
\frac{1 \text { yard }}{1 \text { meter }}=\frac{3600}{3937} \text {. }
$$

All capacities are based on the equivalent 1 liter equals 1.000028 cubic decimeters. $^{3}$ The decimeter is equal to 3.937 inches in accordance with the legal equivalent of the meter given above. The gallon referred to in the tables is the United States gallon of 231 cubic inches. The bushel is the United States bushel of 2150.42 cubic inches. These units must not be confused with the British units of the same name which differ from those used in the United States. The British gallon (277.42 cubic inches) is approximately 20 percent larger, and the British bushel (2 219.36 cubic inches) is 3 percent larger than the corresponding units used in this country.

All weights are derived from the International Kilogram, as authorized in the Mendenhall Order of April 5, 1893. The relation used is 1 avoirdupois pound= 453.5924277 grams.

\section{FUNDAMENTAL EQUIVALENTS}

In its original conception the meter was the fundamental unit of the metric system, and all units of length and capacity were to be derived directly from the meter which was intended to be equal to one ten-millionth of the earth's quadrant. Furthermore, it was originally planned that the unit of mass, the kilogram, should be identical with the mass of a cubic decimeter of water at its maximum density. At present, however, the units of length and mass are defined independently of these conceptions.

1 United States Code, title 15, ch. 6, sec. 205 (Revised Statutes, sec. 3570).

2 U. S. Coast and Geodetic Survey Bulletin 26, Apr. 5, 1893.

${ }^{3}$ Formcrly this relation was given as 1 liter $=1.000027$ cubic decimeters. See Guillaume, La Creation du Bureau International des Poids et Measures et son Oeuvre, Paris, 1927, pages 256 to 258 , for reasons for the change. 
The International Prototype Meter is kept at the International Bureau of Weights and Measures. By definition the meter now bears no definite relation to the earth's quadrant, but is the length of a certain platinum-iridium bar when supported in a definitely specified manner, at the temperature of melting ice, and at standard atmospheric pressure (760 millimeters of mercury). The kilogram is independently defined as the mass of a definite platinum-iridium standard, the International Prototype Kilogram, which is also kept at the International Bureau of Weights and Measures. The liter is defined as the volume of a kilogram of water, at standard atmospheric pressure, and at the temperature of its maximum density, approximately $4^{\circ} \mathrm{C}$. The meter is thus the fundamental unit on which are based all metric standards and measurements of length and area, and of volumes derived from linear measurements.

A supplementary definition of the meter in terms of the wavelength of light was adopted provisionally by the Seventh General (International) Conference on Weights and Measures, in 1927. According to this definition, the relation ${ }^{4}$ for red cadmium light waves under specified conditions of temperature, pressure, and humidity is

1 meter $=1553164.13$ wavelengths.

This corresponds to a wavelength (cadmium red) of

$6438.4696 \times 10^{-7}$ millimeter

The kilogram is the fundamental unit on which are based all metric standards of mass. The liter is a secondary or derived unit of capacity or volume. The liter is larger by about 28 parts per million than the cube of the tenth of the meter, i. e., the cubic decimeter- that is, 1 liter $=1.000028$ cubic decimeters.

The conversion tables in this publication which involve the relative length of the yard and meter are based upon the relation:

$$
1 \text { meter }=39.37 \text { inches, }
$$

contained in the act of Congress of $1866 .^{5} \quad$ From this relation it follows that 1 inch $=$ 25.40005 millimeters (approximately).

For many years the simpler relation, 1 inch $=25.4$ millimeters has been used in engineering and industry throughout the civilized world. ${ }^{6}$ This relation has never been made official in the United States by the Congress or in Great Britain by Parliament.

\section{DEFINITIONS OF UNITS}

\subsection{LENGTH}

\section{Fundamental Units}

A meter $(m)$ is a unit of length equal to the distance between the defining lines on the International Prototype Meter when this standard is at the temperature of melting ice $\left(0^{\circ} \mathrm{C}\right)$.

A yard (yd) is a unit of length equal to $\frac{3600}{3937}$ of a meter.

\section{Multiples and Submultiples}

1 kilometer $(\mathrm{km})=1000$ meters.

1 hectometer $(\mathrm{hm})=100$ meters.

1 dekameter $(\mathrm{dkm})=10$ meters.

1 decimeter $(\mathrm{dm})=0.1$ meter.

1 centimeter $(\mathrm{cm})=0.01$ meter.

4 J. R. Benoit, O. Fabry, and A. Perot, Nouvelle détermination du rapport des longueurs d'onde fondamentales avec l'unité métrique. Trav. et Móm. Bur. Int. Poids et Mesures 15, 131 (1913).

5 United States Code, title 15, sec. 205.

Conversion tables (ASA B48.1-1933), computed on the basis of 1 inch $=25.4 \mathrm{~mm}$ exactly, may be obtained from the American Standards Association, New York, N. Y. 
1 millimeter $(\mathrm{mm})=0.001$ meter.

1 micron $(\mu)=0.000001$ meter $=0.001$ millimeter.

1 millimicron $(\mathrm{m} \mu)=0.000000001$ meter $=0.001$ micron.

1 angstrom (A) $\left\{=0.0000001\right.$ millimeter. $^{7}$

1 angstiom $(\mathrm{A})\left\{\begin{array}{lll}=.000 & 1 & \text { micron. } \\ =.1 & \text { millimicron. }\end{array}\right.$

1 statute mile $\left\{\begin{array}{l}=8 \text { furlongs }=320 \text { rods. } \\ =1 \quad 760 \text { yards }=5 \quad 280 \text { feet. }\end{array}\right.$

1 furlong $=1 / 8$ mile $=40$ rods $=220$ yards $=660$ feet.

1 rod $=5 \frac{1}{2}$ yards $=16 \frac{1}{2}$ feet $=25$ links.

1 foot $=1 / 3$ yard $=12$ inches.

1 hand $=4$ inches.

1 inch $=1 / 36$ yard $=1 / 12$ foot.

1 line (button) $=1 / 40$ inch.

1 point (printers) $=0.013837$ inch $=1 / 72$ inch (nearly).

$1 \mathrm{mil}=11000$ inch.

1 chain (Gunter's) $=4$ rods $=22$ yards $=66$ feet $=100$ links.

1 link (Gunter's) $=11$ 100 chain $=7.92$ inches.

1 U. S. nautical mile)

1 sea mile

1 geographical mile $\}=1853.248$ meters $=6080.20$ feet $^{8}$

1 international nautical mile $=1852$ meters $=6076.10333$. . feet $^{8}$

1 fathom $=6$ feet $=8$ spans.

1 $\operatorname{span}=1 / 8$ fathom $=9$ inches.

\section{Fundamental Units}

\subsection{AREA}

A square meter $\left(\mathrm{m}^{2}\right)$ is a unit of area equal to the area of a square the sides of which are 1 meter.

A square yard $\left(\mathrm{yd}^{2}\right)$ is a unit of area equal to the area of a square the sides of which are 1 yard.

\section{Multiples and Submultiples}

1 square kilometer $\left(\mathrm{km}^{2}\right)=1000000$ square meters.

1 hectare (ha), or square hectometer $\left(\mathrm{hm}^{2}\right)=10000$ square meters.

1 are $(\mathrm{a})$, or square dekameter $\left(\mathrm{dkm}^{2}\right)=100$ square meters.

1 centare (ca) $=1$ square meter.

1 square decimeter $\left(\mathrm{dm}^{2}\right)=0.01$ square meter.

1 square centimeter $\left(\mathrm{cm}^{2}\right)=0.0001$ square meter.

1 square millimeter $\left(\mathrm{mm}^{2}\right)=0.000001$ square meter.

1 square mile $\left(\mathrm{mi}^{2}\right)\{=640$ acres $=102400$ square rods.

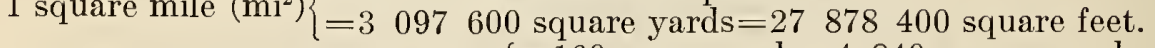

1 acre (acre) $=10$ square chains $\left\{\begin{array}{l}=160 \text { square rods }=4 \quad 840 \text { square yards. } \\ =43 \quad 560 \text { square feet. }\end{array}\right.$

1 square chain $\left(\mathrm{ch}^{2}\right)\left\{\begin{array}{l}=16 \text { square rods }=484 \text { square yards }=4356 \text { square feet. } \\ =10 \quad 000 \text { square links. }\end{array}\right.$

1 square link $\left(\mathrm{li}^{2}\right)\{=0.0001$ square chain $=0.048 \quad 4$ square yard.

1 square $\operatorname{link}\left(\mathrm{l}^{2}\right)\{=0.4356$ square foot $=62.7264$ square inches.

1 square $\operatorname{rod}\left(\mathrm{rd}^{2}\right)=30.25$ square yards $=272.25$ square feet $=625$ square links.

1 square foot $\left(\mathrm{ft}^{2}\right)=1 / 9$ square yard $=144$ square inches.

1 square inch $\left(\right.$ in..$\left.^{2}\right)=1 / 1296$ square yard $=1 / 144$ square foot

\section{Fundamental Units}

\subsection{VOLUME}

A cubic meter $\left(\mathrm{m}^{3}\right)$ is a unit of volume equal to a cube the edges of which are 1 meter. A cubic yard $\left(\mathrm{yd}^{3}\right)$ is a unit of volume equal to a cube the edges of which are 1 yard.

\footnotetext{
7 The angstrom is defined hasically as the unit such that 6438.4696 of these units equal the wavelength of the red radiation of cadmium under speeified conditions.

8 Effeetive July 1,1954 , the lnternational nautical mile as defined ahove was adopted, in lieu of the U.S. nautical mile, for use in the Departments of Defense and Commerce.
} 


\section{Multiples and Submultiples}

1 cubic kilometer $\left(\mathrm{km}^{3}\right)=1000000000$ cubic meters.

1 cubic hectometer $\left(\mathrm{hm}^{3}\right)=1000000$ cubic meters.

1 cubic dekameter $\left(\mathrm{dkm}^{3}\right)=1000$ cubic meters.

1 stere $(\mathrm{s})=1$ cubic meter.

1 cubic decimeter $\left(\mathrm{dm}^{3}\right)=0.001$ cubic meter.

1 cubic centimeter $\left(\mathrm{cm}^{3}\right)=0.000001$ cubic meter $=0.001$ cubic decimeter.

1 cubic millimeter $\left(\mathrm{mm}^{3}\right)=0.000000001$ cubic meter $=0.001$ cubic centimeter.

1 cubic foot $\left(\mathrm{ft}^{3}\right)=1 / 27$ cubic yard.

1 cubic inch (in. $\left.{ }^{3}\right)=1 / 46656$ cubic yard $=1 / 1728$ cubic foot.

1 board foot $(\mathrm{fbm})=144$ cubic inches $=1 / 12$ cubic foot.

1 cord $(\mathrm{cd})=128$ cubic feet.

\subsection{CAPACITY}

\section{Fundamental Units}

A liter (liter) is a unit of capacity equal to the volume occupied by 1 kilogram of pure water at its maximum density (at a temperature of $4^{\circ} \mathrm{C}$, practically) and under the standard atmospheric pressure $($ of $760 \mathrm{~mm}$ ). It is equivalent in volume to 1.000028 cubic decimeters.

A gallon (gal) is a unit of capacity equal to the volume of 231 cubic inches. It is used for the measurement of liquid commodities only.

A bushel (bu) is a unit of capacity equal to the volume of 2150.42 cubic inches. It is used in the measurement of dry commodities only. ${ }^{9}$

\section{Multiples and Submultiples}

1 hectoliter $(\mathrm{hl})=100$ liters.

1 dekaliter $(\mathrm{dkl})=10$ liters.

1 deciliter $(\mathrm{dl})=0.1$ liter .

1 centiliter $(\mathrm{cl})=0.01$ liter.

1 milliliter $(\mathrm{ml})=0.001$ liter $=1.000028$ cubic centimeters.

1 liquid quart (liq $\mathrm{q} t)=1 / 4$ gallon $=57.75$ cubic inches

1 liquid pint (liq $\mathrm{pt})=1 / 8$ gallon $=1 / 2$ liquid quart $=28.875$ cubic inches.

1 gill $(\mathrm{gi})=1 / 32$ gallon $=1 / 4$ liquid pint $=7.21875$ cubic inches.

1 fluid ounce $(\mathrm{fl} \mathrm{oz})=1 / 128$ gallon $=1 / 16$ liquid pint.

1 fluid dram $(\mathrm{fl} \mathrm{dr})=1 / 8$ fluid ounce $=1 / 128$ liquid pint.

$1 \operatorname{minim}(\min$ or $m)=1 / 60$ fluid dram $=1 / 480$ fluid ounce.

1 peck $(\mathrm{pk})=1 / 4$ bushel $=537.605$ cubic inches.

1 dry quart $($ dry qt) $=1 / 32$ bushel $=1 / 8$ peck $=67.200625$ cubic inches.

1 dry pint $($ dry pt $)=1 / 64$ bushel $=1 / 2$ dry quart $=33.6003125$ cubic inches.

1 barrel, for fruits, vegetables, and other dry commodities, other than cranberries ${ }^{10}=7056$ cubic inches $=105$ dry quarts.

1 barrel for cranberries $=5826$ cubic inches. ${ }^{11}$

\section{Fundamental Units}

\subsection{MASS}

A kilogram (kg) is a unit of mass equal to the mass of the International Prototype Kilogram.

A gram (g) is a unit of mass equal to one-thousandth of the mass of the International Prototype Kilogram. gram.

An avoirdupois pound (lb avdp) is a unit of mass equal to 0.4535924277 kilopound.

A troy pound (lb t) is a unit of mass equal to $\frac{5760}{7000}$ of that of the avoirdupois

'This is the so-called stricken or struck bushel. A heaped bushel for apples of 2747.715 cubic inches was established by the U. S. Court of Customs Appeals on Feb. 15, 1912, in United States $v$. Weber (no. 757). A heaped bushel, equivalent to 114 stricken bushels, is also recognized.

10 As fixed by United States standard barrel act (United States Code, title 15, ch. 6, sec. 234).

i1 As computed from dimensions fixed by United States standard barrel act, see above. $408776^{\circ}-56-2$ 
Multiples and Submultiples

1 metric ton $(\mathrm{t})=1000$ kilograms.

1 hectogram $(\mathrm{hg})=100$ grams.

1 dekagr'am $(\mathrm{dkg})=10$ grams.

1 decigram $(\mathrm{dg})=0.1$ gram .

1 centigram $(\mathrm{cg})=0.01$ gram .

1 milligram $(\mathrm{mg})=0.001$ gram .

1 metric carat ${ }^{12}(\mathrm{c})=200$ milligrams $=0.2$ gram.

1 avoirdupois ounce $(\mathrm{oz}$ avdp) $=1 / 16$ avoirdupois pound $=437.5$ grains.

1 avoirdupois $\operatorname{dram}(\mathrm{dr}$ avdp) $=1 / 256$ avoirdupois pound $=1 / 16$ avoirdupois ounce.

1 grain (grain) $=1 / 7000$ avoirdupois pound $=1 / 5760$ troy pound.

1 apothecaries pound ( $\mathrm{lb} a \mathrm{p})=1$ troy pound $=\frac{5760}{7000}$ avoirdupois pound.

1 apothecaries or troy ounce (oz ap or $5, \mathrm{oz} t)=112$ troy pound $=\frac{480}{7000}$ avoirdupois pound $=480$ grains.

1 apothecaries dram ( $\mathrm{dr}$ ap or 3$)=1 / 96$ apothecaries pound $=1 / 8$ apothecaries ounce $=60$ grains.

1 pennyweight $(\mathrm{dwt})=1 / 20$ troy ounce $=24$ grains.

1 apothecaries scruple ( $\mathrm{s}$ ap or $\mathfrak{\theta})=1 / 3$ apothecaries dram $=20$ grains.

1 sliort hundredweight ( $\mathrm{sh} \mathrm{cwt})=100$ avoirdupois pounds.

1 long hundredweight $=112$ avoirdupois pounds.

1 short ton $=2000$ avoirdupois pounds.

1 long ton $=2240$ avoirdupois pounds.

\section{STANDARDS OF MEASUREMENT}

Units of measurement should be distinguished from standards of measurement. Units of length are fixed distances, independent of any other consideration, while length standards are affected by the expansion and contraction resulting from changes of temperature of the material of which the standard is composed. It is therefore necessary to fix upon some temperature at which the distance between the defining lines or end surfaces of the standards shall be equal to the unit. The same is true of standards of capacity, which at some definite temperature contain a given number of units of volume.

The recommended standard temperature for commercial and industrial length standards is $20^{\circ} \mathrm{C}\left(68^{\circ} \mathrm{F}\right)$. Some metric standards, especially those made in Europe until recently, are intended to be correct at $0^{\circ} \mathrm{C}$. In the past some length standards graduated in the customary units have been made to be correct at $62^{\circ} \mathrm{F}\left(16.67^{\circ} \mathrm{C}\right)$.

For measurements of high precision it is also necessary to specify the manner of support of the standards, whether at certain points only or throughout their entire length, and in the case of tapes it is also necessary to give the tension applied to the tape when in use.

In the United States the capacity standards, both metric and customary, are made to hold the specified volumes at $4^{\circ} \mathrm{C}$. Standards of capacity are usually made of brass and the capacity at any other temperature may be computed by the use of the coefficient of cubical expansion of that material usually assumed to be 0.000054 per degree centigrade. In the purchase and sale of liquids a more important consideration than the temperature of the measures is the temperature of the liquid when measured, for the reason that the large coefficient of expansion of many liquids makes the actual mass of a given volume delivered vary considerably with temperature.

While the temperature of a weight does not affect its mass, it is nevertheless important that when two weights are compared in air they both be at the same temperature as the air. If there is a difference between the temperature of the air and the weights, convection currents will be set up and the readings of the balance will be thereby affected. Also, since weights are bouyed up by the surrounding air by amounts dependent upon their volumes, it is desirable that the weights of any

12 See Bureau of Standards Circular C43, The Metric Carat, for a discussion of the metric carat and tables of the relations of the metric carat to the old carat of 205.3 milligrams in use in this country previous to July $1,1913$. 
set be of the same material. If two weights of the same density balance in air of a certain density they will balance in vacuo or in air of a different density.

Brass is the material most widely used for standard weights, although platinum and aluminum are quite commonly used for weights of 1 gram or less. In the absence of any knowledge as to the actual density of weights, those made of brass are assumed to have a density of $8.4 \mathrm{~g} / \mathrm{cm}^{3}$ at $0^{\circ} \mathrm{C}$, while those of platinum and of aluminum are assumed to have densities of 21.5 and $2.7 \mathrm{~g} / \mathrm{cm}^{3}$, respectively.

\section{SPELLING AND ABBREVIATION OF UNITS}

The spelling of the names of units as adopted by the National Bureau of Standards is that given in the list below. The spelling of the metric units is in accordance with that given in the law of July 28, 1866, legalizing the metric system in the United States.

Following the name of each unit in the list below is given the abbreviation which the Bureau has adopted. Attention is particularly called to the following principles:

1. The period is omitted after all abbreviations of units, except where the abbreviation forms an English word.

2. The exponents "2" and "3" are used to signify "square" and "cubic", respectively, instead of the abbreviations "sq" or "cu", which are, however, frequently used in technical literature for the United States customary units. In conformity with this principle the abbreviation for cubic centimeter is " $\mathrm{cm}^{3}$ " (instead of "cc" or "c $\mathrm{cm}$ "). The term "cubic centimeter", as used in chemical work, is, in fact, a nisnomer, since the unit actually used is the "milliliter", of which the correct abbreviation is " $\mathrm{ml}$ " (see p. 1).

3. The use of the same abbreviation for both singular and plural is recommended. This practice is already established in expressing metric units and is in accordance with the spirit and chief purpose of abbreviations.

4. It is also suggested that, unless all the text is printed in capital letters, only small letters be used for abbreviations, except in such case as, A for angstrom, etc., where the use of capital letters is general.

\subsection{LIST OF THE MOST COMMON UNITS OF WEIGHT AND MEASURE AND THEIR} ABBREVIATIONS

\begin{tabular}{|c|c|c|c|c|c|}
\hline Unit & Abbreviation & Unit & Abbreviation & Unit & Abbreviation \\
\hline $\begin{array}{l}\text { acre } \\
\text { angstrom } \\
\text { are } \\
\text { avoirdupois } \\
\text { barrel } \\
\text { board foot } \\
\text { bushel } \\
\text { carat } \\
\text { centare } \\
\text { centigram } \\
\text { centiliter } \\
\text { centimeter } \\
\text { chain } \\
\text { cubic centimeter } \\
\text { cubic decimeter } \\
\text { cubic dekameter } \\
\text { cubic foot } \\
\text { cubic hectometer } \\
\text { cubic inch } \\
\text { cubic kilometer } \\
\text { cubic meter } \\
\text { cubic mile } \\
\text { cubic millimeter } \\
\text { cubic yard } \\
\text { decigram } \\
\text { deciliter } \\
\text { decimeter } \\
\text { decistere } \\
\text { dekagram } \\
\text { dekaliter } \\
\text { dekameter } \\
\text { dekastere } \\
\text { dram } \\
\text { dram, apothacaries }\end{array}$ & 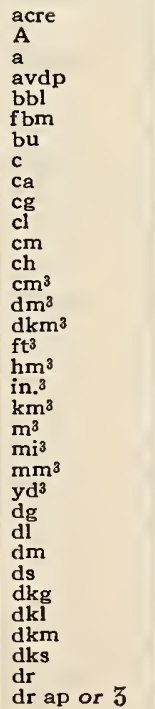 & $\begin{array}{l}\text { dram, avoirdupois } \\
\text { dram, fluid } \\
\text { fathom } \\
\text { foot } \\
\text { furlong } \\
\text { gallon. } \\
\text { grain } \\
\text { gram } \\
\text { hectare } \\
\text { hectogram } \\
\text { hectoliter } \\
\text { hectometer } \\
\text { hogshead } \\
\text { hundredweight } \\
\text { inch } \\
\text { kilogram } \\
\text { kiloliter } \\
\text { kilometer } \\
\text { link } \\
\text { liquid } \\
\text { liter } \\
\text { meter } \\
\text { metric ton } \\
\text { microgram* } \\
\text { microinch } \\
\text { microliter* } \\
\text { micron } \\
\text { mile } \\
\text { milligram } \\
\text { milliliter } \\
\text { millimeter } \\
\text { millimicron } \\
\text { minim } \\
\text { ounce }\end{array}$ & $\begin{array}{l}\text { dr avdp } \\
\text { f dr } \\
\text { fath } \\
\text { ft } \\
\text { fur. } \\
\text { gal } \\
\text { grain } \\
\text { g } \\
\text { ha } \\
\text { hg } \\
\text { hl } \\
\text { hm } \\
\text { hhd } \\
\text { cwt } \\
\text { in. } \\
\mathrm{kg} \\
\text { kl } \\
\mathrm{km} \\
\text { li } \\
\text { liq } \\
\text { liter } \\
\mathrm{m} \\
\mathrm{t} \\
\mu \mathrm{g} \\
\mu \mathrm{in} . \\
\mu \mathrm{l} \\
\mu \\
\mathrm{mi} \\
\mathrm{mg} \\
\mathrm{ml} \\
\mathrm{mm} \\
\mathrm{m} \mu \\
\mathrm{min} \text { or } \mathrm{m} \\
\mathrm{oz}\end{array}$ & $\begin{array}{l}\text { ounce, apothecaries } \\
\text { ounce, avoirdupois } \\
\text { ounce, fluid } \\
\text { ounce, troy } \\
\text { peck } \\
\text { pennyweight } \\
\text { pint } \\
\text { pound } \\
\text { pound, apothecaries } \\
\text { pound, avoirdupois } \\
\text { pound, troy } \\
\text { quart } \\
\text { rod } \\
\text { scruple, apothecaries } \\
\text { square centimeter } \\
\text { square chain } \\
\text { square decimeter } \\
\text { square dekameter } \\
\text { square foot } \\
\text { square hectometer } \\
\text { square inch } \\
\text { square kilometer } \\
\text { square link } \\
\text { square meter } \\
\text { square mile } \\
\text { square millimeter } \\
\text { square rod } \\
\text { square yard } \\
\text { stere } \\
\text { ton } \\
\text { ton, metric } \\
\text { troy } \\
\text { yard }\end{array}$ & $\begin{array}{l}\text { oz ap or } 5 \\
\text { oz avdp } \\
\text { f oz } \\
\text { oz t } \\
\text { pk } \\
\text { dwt } \\
\text { pt } \\
\text { lb } \\
\text { lb ap } \\
\text { lb avdp } \\
\text { lb t } \\
\text { qt } \\
\text { rd } \\
\text { s ap or } B \\
\mathrm{~cm}^{2} \\
\mathrm{ch}^{2} \\
\mathrm{dm}^{2} \\
\mathrm{dkm}^{2} \\
\mathrm{ft}^{2} \\
\mathrm{hm}^{2} \\
\mathrm{in}^{2} \\
\mathrm{~km}^{2} \\
\mathrm{li}^{2} \\
\mathrm{~m}^{2} \\
\mathrm{mi}^{2} \\
\mathrm{~mm}^{2} \\
\mathrm{rd}^{2} \\
\mathrm{yd}^{2} \\
\mathrm{~s} \\
\mathrm{ton}^{2} \\
\mathrm{t} \\
\mathrm{t} \\
\mathrm{yd}^{2}\end{array}$ \\
\hline
\end{tabular}

*The abbreviations $\gamma$ and $\lambda$ for microgram and microliter, respectively, are advocated by some authorities. 
6. TABLES OF INTERRELATION

6.1. UNITS OF

\begin{tabular}{|c|c|c|c|c|c|}
\hline Units & Inches & Links & Feet & Yards & Rods \\
\hline 1 inch & 1 & 0.126263 & 0.0833333 & 0.0277778 & 0.00505051 \\
\hline 1 link & 7.92 & 1 & 0.66 & 0.22 & 0.04 \\
\hline 1 foot & 12 & 1.515152 & 1 & 0.333333 & 0.0606061 \\
\hline 1 yard & 36 & 4.54545 & 3 & 1 & 0.181818 \\
\hline $1 \mathrm{rod}$ & 198 & 25 & 16.5 & 5.5 & $\mathbf{1}$ \\
\hline 1 chain $=$ & 792 & 100 & 66 & 22 & 4 \\
\hline 1 mile & 63360 & 8000 & 5280 & 1760 & 320 \\
\hline 1 centimeter $=$ & 0.3937 & 0.04970960 & 0.03280833 & 0.010936111 & 0.001988384 \\
\hline 1 meter $=$ & 39.37 & 4.970960 & 3. 280833 & 1.0936111 & 0.1988384 \\
\hline
\end{tabular}

6.2. UNITS OF

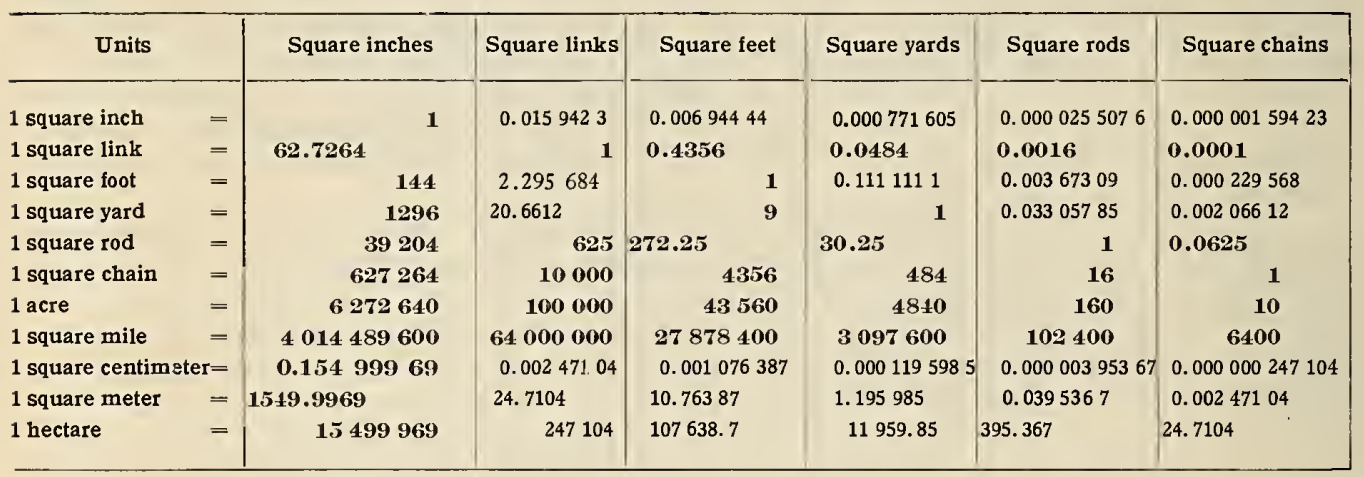

6.3. UNITS OF

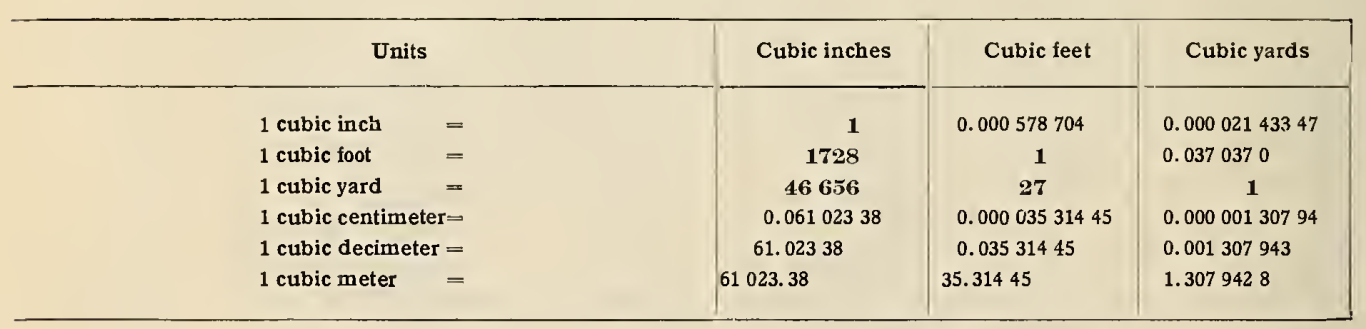

6.4. UNITS OF CAPACITY

\begin{tabular}{|c|c|c|c|c|c|}
\hline Units & Minims & Fluid drams & Fluid ounces & Gills & Liquid pints \\
\hline $1 \operatorname{minim}=$ & $\mathbf{1}$ & 0.0166667 & 0.00208333 & 0.000520833 & 0.000130208 \\
\hline 1 fiuid dram $=$ & 60 & $\mathbf{1}$ & 0.125 & 0.03125 & 0.0078125 \\
\hline 1 fluid ounce $=$ & 480 & 8 & 1 & 0.25 & 0.0625 \\
\hline 1 gill $=$ & 1920 & 32 & 4 & 1 & 0.25 \\
\hline 1 liquid pint $=$ & 7680 & 128 & 16 & 4 & 1 \\
\hline 1 liquid quart= & 15360 & 256 & 32 & 8 & 2 \\
\hline 1 gallon $=$ & 61440 & 1024 & 128 & 32 & 8 \\
\hline 1 milliliter $=$ & 16.2311 & 0.270518 & 0.0338148 & 0.00845369 & $0.002 \quad 11342$ \\
\hline 1 liter $=$ & 16231.1 & 270.518 & 33.8148 & 8.45369 & 2.11342 \\
\hline 1 cubic inch $=$ & 265.974 & 4.43290 & 0.554113 & 0.138528 & 0.0346320 \\
\hline 1 cubic foot $=$ & 459603.1 & 7660.052 & 957.5065 & 239.3766 & $59.844 \quad 16$ \\
\hline
\end{tabular}


OF UNITS OF MEASUREMENT

\section{LENGTH}

\begin{tabular}{|c|c|c|c|c|}
\hline Chains & Miles & Centimeters & Meters & Units \\
\hline 0.00126263 & 0.0000157828 & 2.540005 & 0.02540005 & $=1$ inch \\
\hline 0.01 & 0.000125 & 20.11684 & 0.2011684 & $=1$ link \\
\hline 0.0151515 & 0.0001893939 & 30.48006 & 0.3048006 & $=1$ foot \\
\hline 0.0454545 & 0.000568182 & 91.44018 & 0.9144018 & $=1$ yard \\
\hline 0.25 & 0.003125 & 502.9210 & 5.029210 & $=1 \mathrm{rod}$ \\
\hline 1 & 0.0125 & 2011.684 & 20.11684 & $=1$ chain \\
\hline 80 & 1 & 160934.72 & 1609.3472 & $=1 \mathrm{mile}$ \\
\hline 0.0004970960 & 0.000006213699 & 1 & 0.01 & $=1$ centimeter \\
\hline 0.04970960 & 0.0006213699 & 100 & 1 & $=1 \mathrm{~meter}$ \\
\hline
\end{tabular}

\section{AREA}

\begin{tabular}{|c|c|c|c|c|c|}
\hline Acres & Square miles & Square centimeters & Square meters & Hectares & Units \\
\hline 0.000000159423 & 0.0000000002491 & 6.451626 & 0.0006451626 & 0.000000064516 & $=1$ square inch \\
\hline 0.00001 & 0.000000015625 & 404.6873 & 0.04046873 & 0.00000404687 & $=1$ square link \\
\hline 0.0000229568 & 0.0000000358701 & 929.0341 & 0.09290341 & 0.00000929034 & $=1$ square foot \\
\hline 0.000206612 & 0.000000322831 & 8361.307 & 0.8361307 & 0.0000836131 & $=1$ square yard \\
\hline 0.00625 & 0.000009765625 & 252929.5 & 25.29295 & 0.002529295 & $=1$ square rod \\
\hline 0.1 & 0.00015625 & 4046873 & 404.6873 & 0.0404687 & $=1$ square chain \\
\hline $\mathbf{1}$ & 0.0015625 & 40468726 & 4046.873 & 0.404687 & $=1$ acre \\
\hline 610 & 1 & 25899984703 & 2589998 & 258.9998 & $=1$ square mile \\
\hline 0.0000000247104 & 0.00000000003861006 & 1 & 0.0001 & 0.00000001 & $=1$ square centimeter \\
\hline 0.000247104 & 0.0000003861006 & 10000 & 1 & 0.0001 & $=1$ square meter \\
\hline 2.47104 & 0.003861006 & 100000000 & 10000 & & $=1$ hectare \\
\hline
\end{tabular}

\section{VOLUME}

\begin{tabular}{|c|c|c|c|}
\hline Cubic centimeters & Cubic decimeters & Cubic meters & Units \\
\hline 16.387162 & 0.01638716 & 0.00001638716 & $=1 \mathrm{cubic}$ inch \\
\hline 28317.016 & 28.317016 & 0.028317016 & $=1$ cubic foot \\
\hline 764559.4 & 764.5594 & 0.7645594 & $=1$ cubic yard \\
\hline 1 & 0.001 & 0.000001 & $=1$ cubic centimeter \\
\hline 1000 & 1 & 0.001 & $=1$ cubic decimeter \\
\hline 1000000 & 1000 & 1 & $=1$ cubic meter \\
\hline
\end{tabular}

\section{LIQUID MEASURE}

\begin{tabular}{|c|c|c|c|c|c|c|}
\hline Liquid quarts & Gallons & Milliliters & Liters & Cubic inches & Cubic feet & Units \\
\hline $0.000 \quad 065 \quad 104$ & $0.000 \quad 016 \quad 276$ & 0.0616102 & $0.000 \quad 061 \quad 6102$ & 0.00375977 & $\begin{array}{llll}0.000 & 002 & 175 & 79\end{array}$ & $=1$ minimum \\
\hline 0.00390625 & 0.000976562 & 3.69661 & 0.00369661 & 0.225586 & $0.000 \quad 130 \quad 547$ & $=1$ fluid dram \\
\hline 0.03125 & 0.0078125 & 29.5729 & 0.0295729 & 1. 80469 & 0. $001 \quad 044 \quad 38$ & $=1$ fluid ounce \\
\hline 0.125 & 0.03125 & 118.292 & 0.118292 & 7.21875 & $0.004 \quad 177 \quad 52$ & $=1$ gill \\
\hline 0.5 & 0.125 & 473.166 & $0.473 \quad 166$ & 28.875 & 0.0167101 & $=1$ liquid pint \\
\hline 1 & 0.25 & 946.332 & 0.946332 & 57.75 & 0.0334201 & $=1$ liquid quart \\
\hline 4 & 1 & 3785.329 & $3.785 \quad 329$ & 231 & 0.1336806 & $=1$ gallon \\
\hline $\begin{array}{llll}0.001 & 056 & 71\end{array}$ & $0.000264 \quad 178$ & 1 & 0.001 & $\begin{array}{llll}0.061 & 025 & 1\end{array}$ & $0.000 \quad 035 \quad 315 \quad 4$ & $=1$ milliliter \\
\hline 1.05671 & $0.264 \quad 178$ & 1000 & 1. & 61.0251 & $0.035 \quad 315 \quad 4$ & $=1$ liter \\
\hline 0.0173160 & 0. 00432900 & 16.3867 & 0.0163867 & 1 & $0.000 \quad 578 \quad 703 \quad 7$ & $=1$ cubic inch \\
\hline 29.92208 & 7.4805195 & 28316.22 & 28.31622 & 1728 & & $=1$ cubic foot \\
\hline
\end{tabular}


6.5. UNITS OF CAPACITY

\begin{tabular}{|c|c|c|c|c|}
\hline Units & Dry pints & Dry quarts & Pecks & Bushels \\
\hline 1 dry pint $=$ & $\mathbf{1}$ & 0.5 & 0.0625 & 0.015625 \\
\hline $1 \mathrm{dry}$ quar $\mathrm{t}=$ & $\mathbf{2}$ & 1 & 0.125 & 0.03125 \\
\hline 1 peck $=$ & 16 & 8 & $\mathbf{1}$ & 0.25 \\
\hline 1 bushel $=$ & 64 & 32 & 4 & 1 \\
\hline 1 liter = & 1.81621 & 0.908103 & 0.113513 & 0.028378 \\
\hline 1 dekaliter $=$ & 18.1621 & 9.08103 & 1.13513 & 0.28378 \\
\hline 1 cubic inch $=$ & 0.0297616 & 0.0148808 & 0.00186010 & 0.000465025 \\
\hline 1 cubic foot $=$ & 51.428093 & 25.714047 & 3.2142558 & 0.80356395 \\
\hline
\end{tabular}

\subsection{UNITS OF MASS NOT GREATER}

\begin{tabular}{|c|c|c|c|c|c|c|c|}
\hline Units & & Grains & $\begin{array}{l}\text { Apothecaries } \\
\text { scruples }\end{array}$ & Pennyweights & $\begin{array}{l}\text { Avoirdupois } \\
\text { drams }\end{array}$ & $\begin{array}{l}\text { Apothecaries } \\
\text { drams }\end{array}$ & $\begin{array}{l}\text { Avoirdupois } \\
\text { ounces }\end{array}$ \\
\hline 1 grain & $=$ & $\mathbf{1}$ & 0.05 & 0.04166667 & 0.03657143 & 0.0166667 & 0.00228571 \\
\hline 1 apoth. scruple & $=$ & 20 & 1 & 0.8333333 & 0.7314286 & 0.333333 & 0.0457143 \\
\hline 1 pennyweight & $=$ & 24 & 1.2 & 1 & 0.8777143 & 0.4 & 0.0548571 \\
\hline 1 avdp. dram & $=$ & 27.34375 & 1.3671875 & 1.139323 & 1 & 0.4557292 & 0.0625 \\
\hline 1 apoth. dram & $=$ & 60 & 3 & 2.5 & 2.194286 & 1 & 0.1371429 \\
\hline 1 avdp. oz. & $=$ & 437.5 & 21.875 & 18.22917 & 16 & 7.29167 & 1 \\
\hline 1 apoth. or troy oz. & & 480 & 24 & 20 & 17.55428 & 8 & 1.0971429 \\
\hline 1 apoth. or troy lb. & & 5760 & 288 & 240 & 210.6514 & 96 & 13.165714 \\
\hline 1 avdp. lb. & $=$ & 7000 & 350 & 291.6667 & 256 & 116.6667 & 16 \\
\hline 1 milligram & $=$ & 0.015432356 & 0.000771618 & 0.0006430148 & 0.0005643833 & 0.0002572059 & 0.00003527396 \\
\hline 1 gram & $=$ & 15.432356 & 0.771618 & 0.64301485 & 0.5643833 & 0.2572059 & 0.03527396 \\
\hline 1 kilogram & $=$ & 15432.356 & 771.6178 & 643.01485 & 564.38332 & 257.20594 & 35.27396 \\
\hline
\end{tabular}

\subsection{UNITS OF MASS NOT LESS}

\begin{tabular}{|c|c|c|c|c|c|}
\hline \multicolumn{2}{|c|}{ Units } & \multirow{2}{*}{$\underbrace{\begin{array}{c}\text { Avoirdupois } \\
\text { ounces }\end{array}}_{1}$} & \multirow{2}{*}{$\begin{array}{c}\begin{array}{c}\text { Avoirdupois } \\
\text { pounds }\end{array} \\
0.0625\end{array}$} & \multirow{2}{*}{$\begin{array}{c}\begin{array}{c}\text { Short hundred- } \\
\text { weights }\end{array} \\
0.000625\end{array}$} & \multirow{2}{*}{$\begin{array}{c}\text { Short tons } \\
0.00003125\end{array}$} \\
\hline 1 avoirdupois & $=$ & & & & \\
\hline 1 avoirdupois & $=$ & 16 & 1 & 0.01 & 0.0005 \\
\hline 1 short hundr & $t=$ & 1600 & 100 & 1 & 0.05 \\
\hline 1 short ton & $=$ & 32000 & 2000 & 20 & 1 \\
\hline 1 long ton & $=$ & 35840 & 2240 & 22.4 & 1.12 \\
\hline 1 kilogram & $=$ & 35. 273957 & 2.20462234 & 0.022046223 & 0.0011023112 \\
\hline 1 metric ton & $=$ & 35273.957 & 2204.62234 & 22.046223 & 1.1023112 \\
\hline
\end{tabular}


DRY MEASURE

\begin{tabular}{|c|c|c|c|c|}
\hline Liters & Dekaliters & Cubic inches & Cubic feet & Units \\
\hline $\begin{array}{c}0.550598 \\
1.101 \quad 197 \\
8.80957 \\
35.2383 \\
1 \\
10 \\
0.0163867 \\
28.31622\end{array}$ & 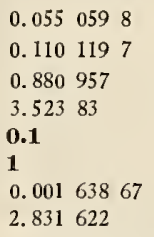 & 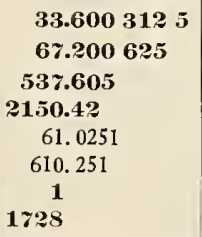 & $\begin{array}{llll}0.019 & 444 & 63 \\
0.038 & 889 & 25 \\
0.311 & 114 & \\
1.244 & 456 & \\
0.035 & 315 & 4 & \\
0.353 & 154 & & \\
0.000 & 578 & 703 & 7 \\
1 & & & \end{array}$ & $\begin{array}{l}=1 \text { dry pint } \\
=1 \text { dry quart } \\
=1 \text { peck } \\
=1 \text { bushel } \\
=1 \text { liter } \\
=1 \text { dekaliter } \\
=1 \text { cubic inch } \\
=1 \text { cubic foot }\end{array}$ \\
\hline
\end{tabular}

\section{THAN POUNDS AND KILOGRAMS}

\begin{tabular}{|c|c|c|c|c|c|c|}
\hline $\begin{array}{c}\text { Apothecaries or } \\
\text { troy ounces }\end{array}$ & $\begin{array}{c}\text { Apothecaries or } \\
\text { troy pounds }\end{array}$ & $\begin{array}{l}\text { Avoirdupois } \\
\text { pounds }\end{array}$ & Milligrams & Grams & Kilograms & Units \\
\hline 0.00208333 & 0.0001736111 & 0.0001428571 & 64.798918 & 0.064798918 & 0.0000647989 & $=1$ grain \\
\hline 0.0416667 & 0.003472222 & 0.002857143 & 1295.9784 & 1.2959784 & 0.001295978 & $=1$ apoth. scruple \\
\hline 0.05 & 0.004166667 & 0.003428571 & 1555.1740 & 1.5551740 & 0.001555174 & $=1$ pennyweight \\
\hline 0.056966146 & 0.0047471788 & 0.00390625 & 1771.8454 & 1.7718454 & 0.001771845 & $=1$ avdp. dram \\
\hline 0.125 & 0.010416667 & 0.008571429 & 3887.9351 & 3.8879351 & 0.003887935 & $=1$ apoth. dram \\
\hline 0.9114583 & 0.075954861 & 0.0625 & 28349.527 & 28.349527 & 0.02834953 & $=1$ avdp. ounce \\
\hline $\mathbf{1}$ & 0.08333333 & 0.06857143 & 31103.481 & 31.103481 & 0.03110348 & $=1$ apoth. or troy ounce \\
\hline 12 & $\mathbf{1}$ & 0.8228571 & 373241.77 & 373.24177 & 0.37324177 & $=1$ a poth. or troy pound \\
\hline 14.583333 & 1.2152778 & 1 & 4.53592 .4277 & 453.5924277 & 0.4535924277 & $=1$ avdp. pound \\
\hline 0.00003215074 & 0.00000267923 & 0.00000220462 & $\mathbf{1}$ & 0.001 & 0.000001 & $=1$ milligram \\
\hline 0.03215074 & 0.00267923 & 0.00220462 & 1000 & 1 & 0.001 & $=1 \mathrm{gram}$ \\
\hline 32.150742 & 2.6792285 & 2.204622341 & 1000000 & 1000 & 1 & $=1 \mathrm{kilogram}$ \\
\hline
\end{tabular}

\section{THAN AVOIRDUPOIS OUNCES}

\begin{tabular}{|l|c|l|l}
\hline \multicolumn{1}{|c|}{ Long tons } & Kilograms & Metric tons & Units \\
\hline 0.00002790179 & 0.02834953 & 0.00002834953 & $=1$ avoirdupois ounce \\
0.0004464286 & $\mathbf{0 . 4 5 3 5 9 2 4 2 7} 7$ & 0.00045359243 & $=1$ avoirdupois pound \\
0.04464286 & 45.359243 & 0.045359243 & $=1$ short hundredweight \\
0.8928571 & 907.18486 & 0.90718486 & $=1$ short ton \\
1 & 1016.04704 & 1.01604704 & $=1$ long ton \\
0.0009842064 & $\mathbf{1}$ & $\mathbf{0 . 0 0 1}$ & $=1$ milogram \\
0.98420640 & $\mathbf{1 0 0 0}$ & $\mathbf{1}$ & \\
\hline
\end{tabular}




\section{COMPARISON OF METRIC AND CUSTOMARY UNITS FROM 1 TO 9}

\subsection{LENGTH}

\begin{tabular}{|c|c|c|c|c|c|}
\hline $\begin{array}{l}\text { Inches } \\
\text { (in.) }\end{array}$ & $\begin{array}{l}\text { Milli- } \\
\text { meters } \\
(\mathrm{mm})\end{array}$ & $\begin{array}{l}\text { Meters } \\
(\mathrm{m})\end{array}$ & $\begin{array}{l}\text { Meters } \\
(\mathrm{m})\end{array}$ & $\begin{array}{l}\text { Meters } \\
(\mathrm{m})\end{array}$ & $\underset{(\mathrm{mi})}{\text { U.S. miles }} \underset{(\mathrm{km})}{\text { Kilometers }}$ \\
\hline & $\begin{array}{l}25.4001 \\
50.8001 \\
76.2002 \\
101.6002\end{array}$ & $\begin{array}{l}\mathbf{1}=0.304801 \\
2=0.609601 \\
3=0.914402 \\
4=1.219202\end{array}$ & 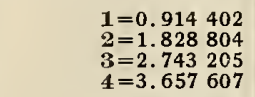 & $\begin{array}{l}1=5.02921 \\
\mathbf{2}=10.05842 \\
3=15.08763 \\
4=20.11684\end{array}$ & $\begin{array}{l}\mathbf{1}=1.609347 \\
\mathbf{2}=3.218694 \\
\mathbf{3}=\mathbf{4} .828042 \\
\mathbf{4}=6.437 \\
\mathbf{3} 89\end{array}$ \\
\hline & $\begin{array}{l}127.0003 \\
152.4003 \\
177.8004 \\
203.2004 \\
228.6005\end{array}$ & 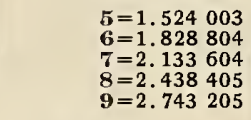 & $\begin{array}{l}\mathbf{5}=4.572009 \\
\mathbf{6}=5.486 \\
\mathbf{7}=6.400 \\
\mathbf{8}=7.315 \\
\mathbf{9}=\mathbf{8} .229616\end{array}$ & $\begin{array}{l}\mathbf{5}=25.14605 \\
\mathbf{6}=30.17526 \\
\mathbf{7}=35.20447 \\
8=40.23368 \\
9=45.26289\end{array}$ & $\begin{array}{l}\mathbf{5}=8.046736 \\
\mathbf{6}=9.656083 \\
\mathbf{7}=11.265 \\
8=12.874 \\
\mathbf{9}=14.484 \\
\end{array}$ \\
\hline $\begin{array}{l}0.0393 \\
0.0787 \\
0.1181 \\
0.1574\end{array}$ & & $\begin{array}{r}3.28083=1 \\
6.56167=2 \\
9.84250=3 \\
13.12333=4\end{array}$ & $\begin{array}{l}1.093611=1 \\
2.187222=2 \\
3.280833=3 \\
4.374444=4\end{array}$ & $\begin{array}{l}0.198838=1 \\
0.397677=2 \\
0.596515=3 \\
0.795354=4\end{array}$ & $\begin{array}{l}0.621370=1 \\
1.242740=2 \\
1.864110=3 \\
2.485480=4\end{array}$ \\
\hline $\begin{array}{l}0.1968 \\
0.2362 \\
0.2755 \\
0.3149 \\
0.3543\end{array}$ & & $\begin{array}{l}16.40417=5 \\
19.68500=6 \\
22.96583=7 \\
26.24667=8 \\
29.52750=9\end{array}$ & $\begin{array}{l}5.468056=5 \\
6.561667=6 \\
7.655278=7 \\
8.748889=8 \\
9.842500=9\end{array}$ & $\begin{array}{l}0.994192=5 \\
1.193030=6 \\
1.391869=7 \\
1.590707=8 \\
1.789545=9\end{array}$ & $\begin{array}{l}3.106850=5 \\
3.728220=6 \\
4.349590=7 \\
4.970960=8 \\
5.592330=9\end{array}$ \\
\hline
\end{tabular}

7.2. AREA

\begin{tabular}{|c|c|c|c|c|c|}
\hline $\begin{array}{l}\text { Square } \\
\text { inches } \\
\left(\text { in. } .^{2}\right)\end{array}$ & $\begin{array}{l}\text { Square } \\
\text { centimeters } \\
\left(\mathrm{cm}^{2}\right)\end{array}$ & $\begin{array}{l}\text { Square } \\
\text { meters } \\
\left(\mathrm{m}^{2}\right)\end{array}$ & $\begin{array}{l}\text { Square } \\
\text { yards } \\
\left(\mathrm{yd}^{2}\right)\end{array}$ & $\begin{array}{l}\text { Hectares } \\
\text { (ha) }\end{array}$ & $\begin{array}{cc}\text { Square } & \text { Square } \\
\text { miles } & \text { kilometers } \\
\left(\mathrm{mi}^{2}\right) & \left(\mathrm{km}^{2}\right)\end{array}$ \\
\hline & $\begin{array}{l}\mathbf{1}=6.452 \\
\mathbf{2}=12.903 \\
3=19.355 \\
\mathbb{4}=25.807\end{array}$ & $\begin{array}{l}1=0.09290 \\
2=0.18581 \\
3=0.27871 \\
4=0.37161\end{array}$ & $\begin{array}{l}\mathbf{1}=0.8361 \\
\mathbf{2}=1.6723 \\
\mathbf{3}=2.5084 \\
4=3.3445\end{array}$ & $\begin{array}{l}1=0.4047 \\
2=0.8094 \\
3=1.2141 \\
4=1.6187\end{array}$ & $\begin{array}{l}1=2.5900 \\
2=5.1800 \\
3=7.7700 \\
4=10.3600\end{array}$ \\
\hline & $\begin{array}{l}\mathbf{5}=32.258 \\
\mathbf{6}=38.710 \\
7=45.161 \\
8=51.613 \\
9=58.065\end{array}$ & 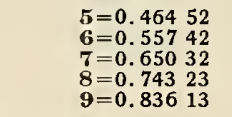 & $\begin{array}{l}5=4.1807 \\
6=5.0168 \\
7=5.8529 \\
8=6.6890 \\
9=7.5252\end{array}$ & $\begin{array}{l}\mathbf{5}=2.0234 \\
\mathbf{6}=2.4281 \\
\mathbf{7}=2.8328 \\
8=3.2375 \\
\mathbf{9}=3.6422\end{array}$ & $\begin{array}{l}\mathbf{5}=12.9500 \\
6=15.5400 \\
7=18.1300 \\
8=20.7200 \\
9=23.3100\end{array}$ \\
\hline \multicolumn{2}{|c|}{$\begin{array}{l}0.15500=1 \\
0.31000=2 \\
0.46500=3 \\
0.62000=4\end{array}$} & $\begin{array}{l}10.764=1 \\
21.528=2 \\
32.292=3 \\
43.055=4\end{array}$ & $\begin{array}{l}1.1960=1 \\
2.3920=2 \\
3.5880=3 \\
4.7839=4\end{array}$ & $\begin{array}{l}2.471=1 \\
4.942=2 \\
7.413=3 \\
9.884=4\end{array}$ & $\begin{array}{l}0.3861=1 \\
0.7722=2 \\
1.1583=3 \\
1.5444=4\end{array}$ \\
\hline \multicolumn{2}{|c|}{$\begin{array}{l}0.77500=5 \\
0.93000=6 \\
1.08500=7 \\
1.24000=8 \\
1.39500=9\end{array}$} & $\begin{array}{l}53.819=5 \\
64.583=6 \\
75.347=7 \\
86.111=8 \\
96.875=9\end{array}$ & $\begin{array}{r}5.9799=5 \\
7.1759=6 \\
8.3719=7 \\
9.5679=8 \\
10.7639=9\end{array}$ & $\begin{array}{l}12.355=5 \\
14.826=6 \\
17.297=7 \\
19.768=8 \\
22.239=9\end{array}$ & $\begin{array}{l}1.9305=5 \\
2.3166=6 \\
2.7027=7 \\
3.0888=8 \\
3.4749=9\end{array}$ \\
\hline
\end{tabular}

7.3. VOLUME

\begin{tabular}{|c|c|c|c|c|c|c|}
\hline $\begin{array}{l}\text { Cubic } \\
\text { inches } \\
\left.\text { (in. }{ }^{3}\right)\end{array}$ & $\begin{array}{c}\text { Cubic } \\
\text { centimeters } \\
\left(\mathrm{cm}^{3}\right)\end{array}$ & $\begin{array}{c}\text { Cubic } \\
\text { meters } \\
\left(\mathrm{m}^{3}\right)\end{array}$ & $\begin{array}{c}\text { Cubic } \\
\text { meters } \\
\left(\mathrm{m}^{3}\right)\end{array}$ & $\begin{array}{l}\text { Liters } \\
\text { (liter) }\end{array}$ & $\begin{array}{l}\text { Cubic } \\
\text { feet } \\
\left(\mathrm{ft}^{3}\right)\end{array}$ & $\begin{array}{l}\text { Liters } \\
\text { (liter) }\end{array}$ \\
\hline & $\begin{array}{l}\mathbf{1}=16.3872 \\
\mathbf{2}=32.7743 \\
3=49.1615 \\
4=65.5486\end{array}$ & $\begin{array}{l}1=0.028317 \\
2=0.056634 \\
3=0.084951 \\
4=0.113268\end{array}$ & $\begin{array}{l}1=0.7646 \\
2=1.5291 \\
3=2.2937 \\
4=3.0582\end{array}$ & 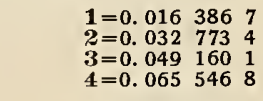 & & $\begin{array}{l}\mathbf{1}=28.316 \\
\mathbf{2}=56.632 \\
\mathbf{3}=84.949 \\
\mathbf{4}=113.265\end{array}$ \\
\hline & $\begin{array}{l}5=81.9358 \\
6=98.3230 \\
7=114.7101 \\
8=131.0973 \\
9=147.4845\end{array}$ & $\begin{array}{l}\mathbf{5}=0.141585 \\
\mathbf{6}=0.169 \\
\mathbf{7}=0.198 \\
8=0.226 \\
\mathbf{9}=0.254 \\
\mathbf{8}\end{array}$ & $\begin{array}{l}5=3.8228 \\
6=4.5874 \\
7=5.3519 \\
8=6.1165 \\
9=6.8810\end{array}$ & 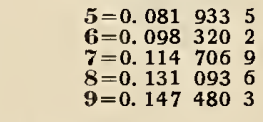 & & $\begin{array}{l}5=141.581 \\
6=169.897 \\
7=198.214 \\
8=226.530 \\
9=254.846\end{array}$ \\
\hline $\begin{array}{l}0.0610 \\
0.1220 \\
0.1830 \\
0.244\end{array}$ & $\begin{array}{l}02=1 \\
05=2 \\
07=3 \\
09=4\end{array}$ & $\begin{array}{r}35.314=1 \\
70.629=2 \\
105.943=3 \\
141.258=4\end{array}$ & $\begin{array}{l}1.3079=1 \\
2.6159=2 \\
3.9238=3 \\
5.2318=4\end{array}$ & $\begin{array}{r}61.025=\mathbf{1} \\
122.050=\mathbf{2} \\
183.075=\mathbf{3} \\
244.100=\mathbf{4}\end{array}$ & $\begin{array}{l}0.035 \\
0.070 \\
0.105 \\
0.141 \\
0\end{array}$ & $\begin{array}{l}315=1 \\
631=2 \\
946=3 \\
262=4\end{array}$ \\
\hline $\begin{array}{l}0.3051 \\
0.3661 \\
0.4271 \\
0.4881 \\
0.549\end{array}$ & $\begin{array}{l}12=5 \\
14=6 \\
16=7 \\
19=8 \\
21=9\end{array}$ & $\begin{array}{l}176.572=5 \\
211.887=6 \\
247.201=7 \\
282.516=8 \\
317.830=9\end{array}$ & $\begin{array}{r}6.5397=5 \\
7.8477=6 \\
9.1556=7 \\
10.4635=8 \\
11.7715=9\end{array}$ & $\begin{array}{l}305.125=5 \\
366.151=6 \\
427.176=7 \\
488.207=8 \\
549.226=9\end{array}$ & $\begin{array}{l}0.176 \\
0.211 \\
0.247 \\
0.282 \\
0.317 \\
0\end{array}$ & $\begin{array}{l}577=5 \\
893=6 \\
208=7 \\
524=8 \\
839=9\end{array}$ \\
\hline
\end{tabular}


7.4. CAPACITY-LIQUID MEASURE

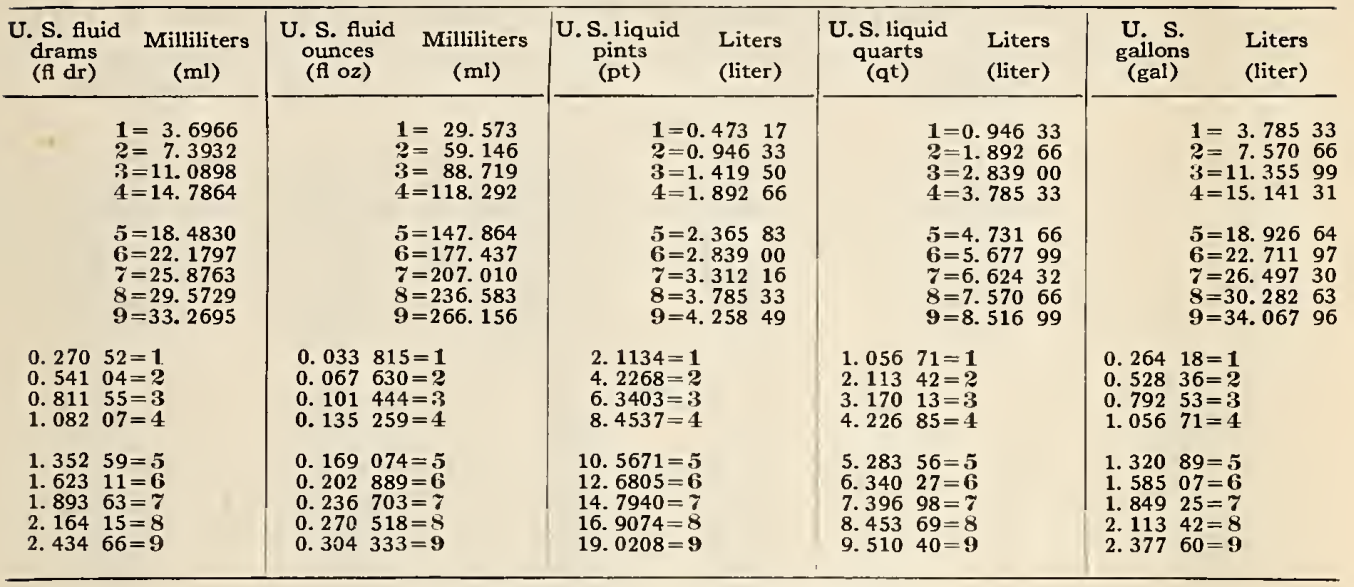

\subsection{CAPACITY-DRY MEASURE}

\begin{tabular}{|c|c|c|c|c|}
\hline $\begin{array}{l}\text { U. S. } \\
\text { dry } \\
\text { quarts } \\
\text { (qt) }\end{array}$ & $\begin{array}{l}\text { U. S. } \\
\text { pecks } \\
\text { (pk) }\end{array}$ & $\begin{array}{l}\text { Dekaliters } \\
\text { (dkl) }\end{array}$ & $\begin{array}{l}\text { U. S. } \\
\text { bushels } \\
\text { (bu) }\end{array}$ & $\begin{array}{cc}\text { U.S. } & \text { Hecto- } \\
\text { bushels } & \text { liters per } \\
\text { per acre } & \text { hectare }\end{array}$ \\
\hline $\begin{array}{l}1=1.1012 \\
2=2.2024 \\
3=3.3036 \\
4=4.4048\end{array}$ & $\begin{array}{l}1=8.810 \\
2=17.619 \\
3=26.429 \\
4=35.238\end{array}$ & $\begin{array}{l}\mathbf{1}=0.8810 \\
\mathbf{2}=1.7619 \\
\mathbf{3}=2.6429 \\
\mathbf{4}=3.5238\end{array}$ & $\begin{array}{l}1=0.35238 \\
2=0.70477 \\
3=1.05715 \\
4=1.40953\end{array}$ & $\begin{array}{l}1=0.8708 \\
2=1.7415 \\
3=2.6123 \\
4=3.4830\end{array}$ \\
\hline $\begin{array}{l}\mathbf{5}=\mathbf{5} .5060 \\
\mathbf{6}=6.6072 \\
\mathbf{7}=\mathbf{7} . \mathbf{7 0 8 4} \\
\mathbf{8}=\mathbf{8 . 8 0 9 6} \\
\mathbf{9}=9.9108\end{array}$ & $\begin{array}{l}\boldsymbol{5}=44.048 \\
\mathbf{6}=52.857 \\
\mathbf{7}=61.667 \\
\mathbf{8}=\mathbf{7 0 . 4 7 7} \\
\mathbf{9}=79.286\end{array}$ & $\begin{array}{l}\mathbf{5}=4.4048 \\
\mathbf{6}=5.2857 \\
\mathbf{7}=6.1667 \\
\mathbf{8}=7.0477 \\
\mathbf{9}=7.9286\end{array}$ & $\begin{array}{l}5=1.76191 \\
6=2.11430 \\
7=2.46668 \\
8=2.81906 \\
9=3.171 \quad 45\end{array}$ & $\begin{array}{l}\mathbf{5}=4.3538 \\
\mathbf{6}=5.2245 \\
\mathbf{7}=6.0953 \\
\mathbf{8}=6.9660 \\
\mathbf{9}=\mathbf{7} . \mathbf{8 3 6 8}\end{array}$ \\
\hline $\begin{array}{l}0.9081=1 \\
1.8162=2 \\
2.7243=3 \\
3.6324=4\end{array}$ & $\begin{array}{l}0.11351=1 \\
0.22703=2 \\
0.34054=3 \\
0.45405=4\end{array}$ & $\begin{array}{l}1.1351=1 \\
2.2703=2 \\
3.4054=3 \\
4.5405=4\end{array}$ & $\begin{array}{r}2.8378=1 \\
5.6756=2 \\
8.5135=3 \\
11.3513=4\end{array}$ & $\begin{array}{l}1.1484=1 \\
2.2969=2 \\
3.4453=3 \\
4.5937=4\end{array}$ \\
\hline $\begin{array}{l}4.5405=5 \\
5.4486=6 \\
6.3567=7 \\
7.2648=8 \\
8.1729=9\end{array}$ & $\begin{array}{l}0.56756=5 \\
0.68108=6 \\
0.79459=7 \\
0.90810=8 \\
1.02162=9\end{array}$ & $\begin{array}{r}5.6756=5 \\
6.8108=6 \\
7.9459=7 \\
9.0810=8 \\
10.2162=9\end{array}$ & $\begin{array}{l}14.1891=5 \\
17.0269=6 \\
19.8648=7 \\
22.7026=8 \\
25.5404=9\end{array}$ & $\begin{array}{r}5.7422=5 \\
6.8906=6 \\
8.0390=7 \\
9.1874=8 \\
10.3359=9\end{array}$ \\
\hline
\end{tabular}

\subsection{MASS}

\begin{tabular}{|c|c|c|c|c|c|}
\hline $\begin{array}{l}\text { Grains } \\
\text { (grain) }\end{array}$ & $\begin{array}{l}\text { Apothe- } \\
\text { caries } \\
\text { drams } \\
\text { (dr ap or 3) }\end{array}$ & $\begin{array}{l}\text { Troy } \\
\text { ounces } \\
(o z t)\end{array}$ & $\begin{array}{l}\text { Avoirdu- } \\
\text { pois } \\
\text { ounces } \\
\text { (oz avdp) }\end{array}$ & $\begin{array}{l}\text { Avoirdu- } \\
\text { pois } \\
\text { pounds } \\
\text { (Ib avdp) }\end{array}$ & $\begin{array}{l}\text { Kilo- } \\
\text { grams } \\
\text { (kg) }\end{array}$ \\
\hline $\begin{array}{l}\mathbf{1}=0.064799 \\
\mathbf{2}=0.129598 \\
\mathbf{3}=0.194397 \\
\mathbf{4}=0.259196\end{array}$ & $\begin{array}{l}1=3.8879 \\
2=7.7759 \\
3=11.6638 \\
4=15.5517\end{array}$ & $\begin{array}{l}\mathbf{1}=31.103 \\
2=62.207 \\
3=93.310 \\
4=124.414\end{array}$ & $\begin{array}{l}1=28.350 \\
2=56.699 \\
3=85.049 \\
4=113.398\end{array}$ & \multicolumn{2}{|c|}{ 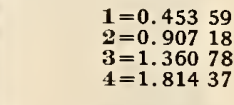 } \\
\hline $\begin{array}{l}\mathbf{5}=0.323 \\
\mathbf{6}=0.388 \\
\mathbf{7}=0.453 \\
\mathbf{8}=0.518 \\
\mathbf{9}=0.583 \\
\mathbf{5} 8 \mathbf{1 9 0}\end{array}$ & $\begin{array}{l}5=19.4397 \\
6=23.3276 \\
7=27.2155 \\
8=31.1035 \\
9=34.9914\end{array}$ & $\begin{array}{l}5=155.517 \\
\mathbf{6}=186.621 \\
7=217.724 \\
8=248.828 \\
9=279.931\end{array}$ & $\begin{array}{l}\boldsymbol{5}=141.748 \\
\mathbf{6}=170.097 \\
\boldsymbol{7}=198.447 \\
\mathbf{8}=225.796 \\
\mathbf{9}=255.146\end{array}$ & \multicolumn{2}{|c|}{ 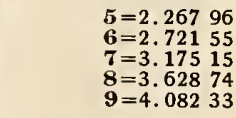 } \\
\hline $\begin{array}{l}15.4324=1 \\
30.8647=2 \\
46.2971=3 \\
61.7294=4\end{array}$ & $\begin{array}{l}0.25721=1 \\
0.51441=2 \\
0.77162=3 \\
1.02882=4\end{array}$ & $\begin{array}{l}0.032151=1 \\
0.064301=2 \\
0.096452=3 \\
0.128603=4\end{array}$ & $\begin{array}{l}0.035274=1 \\
0.070548=2 \\
0.105822=3 \\
0.141096=4\end{array}$ & \multicolumn{2}{|c|}{$\begin{array}{l}2.20462=1 \\
4.40924=2 \\
6.61387=3 \\
8.81849=4\end{array}$} \\
\hline $\begin{array}{r}77.1618=5 \\
92.5941=6 \\
108.0265=7 \\
123.4589=8 \\
138.8912=9\end{array}$ & $\begin{array}{l}1.28603=5 \\
1.54324=6 \\
1.80044=7 \\
2.05765=8 \\
2.31485=9\end{array}$ & $\begin{array}{l}0.160754=5 \\
0.192904=6 \\
0.225055=7 \\
0.257206=8 \\
0.289357=9\end{array}$ & $\begin{array}{l}0.176370=5 \\
0.211644=6 \\
0.246918=7 \\
0.282192=8 \\
0.317466=9\end{array}$ & \multicolumn{2}{|c|}{$\begin{array}{l}11.02311=5 \\
13.22773=6 \\
15.43236=7 \\
17.63698=8 \\
19.84160=9\end{array}$} \\
\hline
\end{tabular}


7.7. COMPARISON OF THE VARIOUS TONS AND POUNDS IN USE IN THE UNITED STATES (FROM 1 TO 9 UNITS)

\begin{tabular}{|c|c|c|c|c|c|}
\hline Troy pounds & Avoirdupois pounds & Kiiograms & Short tons & Long tons & Metric tons \\
\hline $\begin{array}{l}1 \\
\mathbf{2} \\
\mathbf{3} \\
4\end{array}$ & $\begin{array}{l}0.822 \cdot 857 \\
1.64571 \\
2.46857 \\
3.29143\end{array}$ & $\begin{array}{l}0.37324 \\
0.74648 \\
1.11973 \\
1.49297\end{array}$ & $\begin{array}{l}0.00041143 \\
0.00082286 \\
0.00123429 \\
0.00164571\end{array}$ & $\begin{array}{l}0.00036735 \\
0.00073469 \\
0.00110204 \\
0.00146939\end{array}$ & $\begin{array}{l}0.00037324 \\
0.00074648 \\
0.00111973 \\
0.00149297\end{array}$ \\
\hline $\begin{array}{l}\mathbf{5} \\
\mathbf{6} \\
\mathbf{7} \\
8 \\
\mathbf{9}\end{array}$ & $\begin{array}{l}4.11429 \\
4.93714 \\
5.76000 \\
6.58286 \\
740571\end{array}$ & $\begin{array}{l}1.86621 \\
2.23945 \\
2.61269 \\
2.98593 \\
3.35918\end{array}$ & $\begin{array}{l}0.00205714 \\
0.00246857 \\
0.00288000 \\
0.00329143 \\
0.00370286\end{array}$ & $\begin{array}{l}0.00183673 \\
0.00220408 \\
0.00257143 \\
0.00293878 \\
0.00330612\end{array}$ & $\begin{array}{l}0.00186621 \\
0.00223945 \\
0.00261269 \\
0.00298593 \\
0.00335918\end{array}$ \\
\hline $\begin{array}{l}1.21528 \\
2.43056 \\
3.64583 \\
4.86111\end{array}$ & $\begin{array}{l}1 \\
2 \\
3 \\
4\end{array}$ & $\begin{array}{l}0.45359 \\
0.90718 \\
1.36078 \\
1.81437\end{array}$ & $\begin{array}{l}0.0005 \\
0.0010 \\
0.0015 \\
0.0020\end{array}$ & $\begin{array}{l}0.00044643 \\
0.00089286 \\
0.00133929 \\
0.00178571\end{array}$ & $\begin{array}{l}0.00045359 \\
0.00090718 \\
0.00136078 \\
0.00181437\end{array}$ \\
\hline $\begin{array}{r}6.07639 \\
7.29167 \\
8.50694 \\
9.72222 \\
10.93750\end{array}$ & $\begin{array}{l}5 \\
6 \\
7 \\
8 \\
9\end{array}$ & $\begin{array}{l}2.26796 \\
2.72155 \\
3.17515 \\
3.62874 \\
4.08233\end{array}$ & $\begin{array}{l}0.0025 \\
0.0030 \\
0.0035 \\
0.0040 \\
0.0045\end{array}$ & $\begin{array}{l}0.00223214 \\
0.00267857 \\
0.00312500 \\
0.00357143 \\
0.00401786\end{array}$ & $\begin{array}{l}0.00226796 \\
0.00272155 \\
0.00317515 \\
0.00362874 \\
0.00408233\end{array}$ \\
\hline $\begin{array}{r}2.67923 \\
5.35846 \\
8.03769 \\
10.71691\end{array}$ & $\begin{array}{l}2.20462 \\
4.40924 \\
6.61387 \\
8.81849\end{array}$ & $\begin{array}{l}\mathbf{1} \\
\mathbf{2} \\
\mathbf{3} \\
\mathbf{4}\end{array}$ & $\begin{array}{l}0.00110231 \\
0.00220462 \\
0.00330693 \\
0.00440924\end{array}$ & $\begin{array}{l}0.00098421 \\
0.00196841 \\
0.00295262 \\
0.00393683\end{array}$ & $\begin{array}{l}0.001 \\
0.002 \\
0.003 \\
0.004\end{array}$ \\
\hline $\begin{array}{l}13.39614 \\
16.07537 \\
18.75460 \\
21.43383 \\
24.11306\end{array}$ & $\begin{array}{l}11.02311 \\
13.22773 \\
15.43236 \\
17.63698 \\
19.84160\end{array}$ & $\begin{array}{l}5 \\
6 \\
7 \\
8 \\
9\end{array}$ & $\begin{array}{l}0.00551156 \\
0.00661387 \\
0.00771618 \\
0.00881849 \\
0.00992080\end{array}$ & $\begin{array}{l}0.00492103 \\
0.00590524 \\
0.00688944 \\
0.00787365 \\
0.00885786\end{array}$ & $\begin{array}{l}0.005 \\
0.006 \\
0.007 \\
0.008 \\
0.009\end{array}$ \\
\hline $\begin{array}{l}2430.56 \\
4861.11 \\
7291.67 \\
9722.22\end{array}$ & $\begin{array}{l}2000 \\
4000 \\
6000 \\
8000\end{array}$ & $\begin{array}{r}907.18 \\
1814.37 \\
2721.55 \\
3628.74\end{array}$ & $\begin{array}{l}\mathbf{1} \\
\mathbf{2} \\
\mathbf{3} \\
\mathbf{4}\end{array}$ & $\begin{array}{l}0.89286 \\
1.78571 \\
2.67857 \\
3.57143\end{array}$ & $\begin{array}{l}0.90718 \\
1.81437 \\
2.72155 \\
3.62874\end{array}$ \\
\hline $\begin{array}{ll}12 & 152.78 \\
14 & 583.33 \\
17 & 013.89 \\
19 & 444.44 \\
21 & 875.00\end{array}$ & $\begin{array}{l}10000 \\
12000 \\
14000 \\
16000 \\
18000\end{array}$ & $\begin{array}{l}4535.92 \\
5443.11 \\
6350.29 \\
7257.48 \\
8164.66\end{array}$ & $\begin{array}{l}5 \\
\mathbf{6} \\
7 \\
8 \\
9\end{array}$ & $\begin{array}{l}4.46429 \\
5.35714 \\
6.25000 \\
7.14286 \\
8.03571\end{array}$ & $\begin{array}{l}4.53592 \\
5.44311 \\
6.35029 \\
7.25748 \\
8.16466\end{array}$ \\
\hline $\begin{array}{r}2722.22 \\
5444.44 \\
8166.67 \\
10888.89\end{array}$ & $\begin{array}{l}2240 \\
4480 \\
6720 \\
8960\end{array}$ & $\begin{array}{l}1016.05 \\
2032.09 \\
3048.14 \\
4064.19\end{array}$ & $\begin{array}{l}1.12 \\
2.24 \\
3.36 \\
4.48\end{array}$ & $\begin{array}{l}1 \\
2 \\
3 \\
4\end{array}$ & $\begin{array}{l}1.01605 \\
2.03209 \\
3.04814 \\
4.06419\end{array}$ \\
\hline $\begin{array}{l}13611.11 \\
16333.33 \\
19055.56 \\
21777.78 \\
24500.00\end{array}$ & $\begin{array}{l}11200 \\
13440 \\
15680 \\
17920 \\
20160\end{array}$ & $\begin{array}{l}5080.24 \\
6096.28 \\
7112.32 \\
8128.38 \\
9144.42\end{array}$ & $\begin{array}{r}5.60 \\
6.72 \\
7.84 \\
8.96 \\
10.08\end{array}$ & $\begin{array}{l}5 \\
6 \\
7 \\
8 \\
9\end{array}$ & $\begin{array}{l}5.08024 \\
6.09628 \\
7.11232 \\
8.12838 \\
9.14442\end{array}$ \\
\hline $\begin{array}{r}2679.23 \\
5358.46 \\
8037.69 \\
10716.91\end{array}$ & $\begin{array}{l}2204.62 \\
4409.24 \\
6613.87 \\
8818.49\end{array}$ & $\begin{array}{l}1000 \\
2000 \\
3000 \\
4000\end{array}$ & $\begin{array}{l}1.10231 \\
2.20462 \\
3.30693 \\
4.40924\end{array}$ & $\begin{array}{l}0.98421 \\
1.96841 \\
2.95262 \\
3.93683\end{array}$ & $\begin{array}{l}\mathbf{1} \\
\mathbf{2} \\
\mathbf{3} \\
\mathbf{4}\end{array}$ \\
\hline $\begin{array}{ll}13 & 396.14 \\
16 & 075.37 \\
18 & 754.60 \\
21 & 433.83 \\
24 & 113.06\end{array}$ & $\begin{array}{l}11023.11 \\
13227.73 \\
15432.36 \\
17636.98 \\
19841.60\end{array}$ & $\begin{array}{l}5000 \\
6000 \\
7000 \\
8000 \\
9000\end{array}$ & $\begin{array}{l}5.51156 \\
6.61387 \\
7.71618 \\
8.81849 \\
9.92080\end{array}$ & $\begin{array}{l}4.92103 \\
5.905 \quad 24 \\
6.88944 \\
7.87365 \\
8.85786\end{array}$ & $\begin{array}{l}5 \\
6 \\
7 \\
8 \\
9\end{array}$ \\
\hline
\end{tabular}




\section{SPECIAL TABLES}

\section{LENGTH-INCHES AND MILLIMETERS-EQUIVALENTS OF DECIMAL} AND BINARY FRACTIONS OF AN INCH IN MILLIMETERS

From $1 / 64$ to 1 Inch

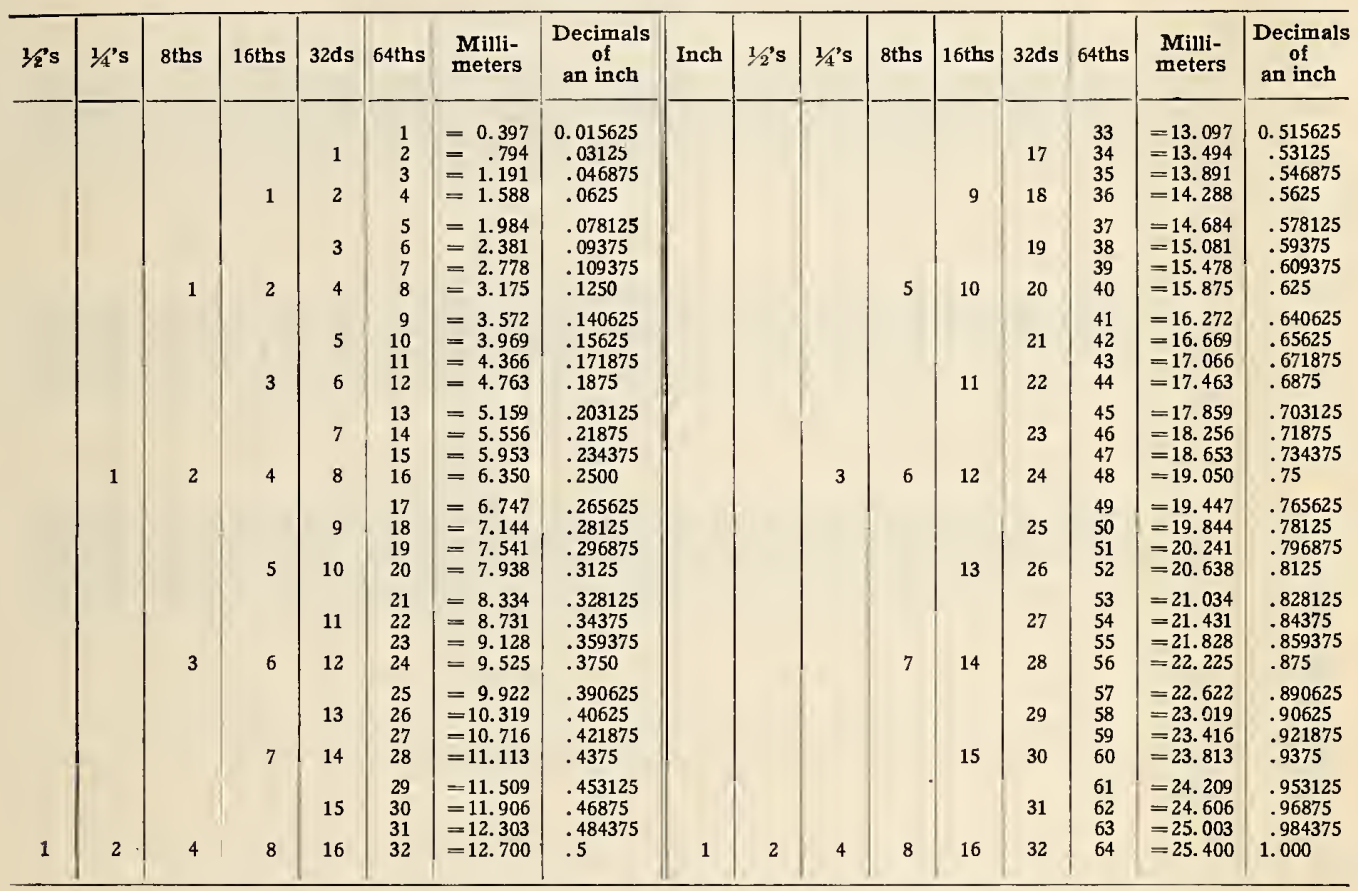

LENGTH-HUNDREDTHS OF AN INCH TO MILLIMETERS

From 1 to 99 Hundredths

\begin{tabular}{|c|c|c|c|c|c|c|c|c|c|c|}
\hline $\begin{array}{l}\text { Hun- } \\
\text { aredths of } \\
\text { an inch }\end{array}$ & $\mathbf{0}$ & 1 & 2 & 3 & 4 & 5 & 6 & 7 & 8 & 9 \\
\hline $\begin{array}{l}10 \\
20 \\
30 \\
40\end{array}$ & $\begin{array}{r}0 \\
2.540 \\
5.080 \\
7.620 \\
10.160\end{array}$ & $\begin{array}{r}0.254 \\
2.794 \\
5.334 \\
7.874 \\
10.414\end{array}$ & $\begin{array}{r}0.508 \\
3.048 \\
5.588 \\
8.128 \\
10.668\end{array}$ & $\begin{array}{r}0.762 \\
3.302 \\
5.842 \\
8.382 \\
10.922\end{array}$ & $\begin{array}{r}1.016 \\
3.556 \\
6.096 \\
8.636 \\
11.176\end{array}$ & $\begin{array}{r}1.270 \\
3.810 \\
6.350 \\
8.890 \\
11.430\end{array}$ & $\begin{array}{r}1.524 \\
4.064 \\
6.604 \\
9.144 \\
11.684\end{array}$ & $\begin{array}{r}1.778 \\
4.318 \\
6.858 \\
9.398 \\
11.938\end{array}$ & $\begin{array}{r}2.032 \\
4.572 \\
7.112 \\
9.652 \\
12.192\end{array}$ & $\begin{array}{r}2.286 \\
4.826 \\
7.366 \\
9.906 \\
12.446\end{array}$ \\
\hline $\begin{array}{l}50 \\
60 \\
70 \\
80 \\
90\end{array}$ & $\begin{array}{l}12.700 \\
15.240 \\
17.780 \\
20.320 \\
22.860\end{array}$ & $\begin{array}{l}12.954 \\
15.494 \\
18.034 \\
20.574 \\
23.114\end{array}$ & $\begin{array}{l}13.208 \\
15.748 \\
18.288 \\
20.828 \\
23.368\end{array}$ & $\begin{array}{l}13.462 \\
16.002 \\
18.542 \\
21.082 \\
23.622\end{array}$ & $\begin{array}{l}13.716 \\
16.256 \\
18.796 \\
21.336 \\
23.876\end{array}$ & $\begin{array}{l}13.970 \\
16.510 \\
19.050 \\
21.590 \\
24.130\end{array}$ & $\begin{array}{l}14.224 \\
16.764 \\
19.304 \\
21.844 \\
24.384\end{array}$ & $\begin{array}{l}14.478 \\
17.018 \\
19.558 \\
22.098 \\
24.638\end{array}$ & $\begin{array}{l}14.732 \\
17.272 \\
19.812 \\
22.352 \\
24.892\end{array}$ & $\begin{array}{l}14.986 \\
17.526 \\
20.066 \\
22.606 \\
25.146\end{array}$ \\
\hline
\end{tabular}

LENGTH-MILLIMETERS TO DECIMALS OF AN INCH

From 1 to 99 Units

\begin{tabular}{|c|c|c|c|c|c|c|c|c|c|c|}
\hline $\begin{array}{l}\text { Milli- } \\
\text { meters }\end{array}$ & 0 & 1 & $\mathbf{2}$ & 3 & 4 & 5 & 6 & 7 & 8 & 9 \\
\hline $\begin{array}{l}10 \\
20 \\
30 \\
40\end{array}$ & $\begin{array}{r}0 \\
0.39370 \\
.78740 \\
1.18110 \\
1.57480\end{array}$ & $\begin{array}{r}0.03937 \\
.43307 \\
.82677 \\
1.22047 \\
1.61417\end{array}$ & $\begin{array}{r}0.07874 \\
.47244 \\
.86614 \\
1.25984 \\
1.65354\end{array}$ & $\begin{array}{r}0.11811 \\
.51181 \\
.90551 \\
1.29921 \\
1.69291\end{array}$ & $\begin{array}{r}0.15748 \\
.55118 \\
.94488 \\
1.33858 \\
1.73228\end{array}$ & $\begin{array}{r}0.19685 \\
.59055 \\
.98425 \\
1.37795 \\
1.77165\end{array}$ & $\begin{array}{r}0.23622 \\
.62992 \\
1.02362 \\
1.41732 \\
1.81102\end{array}$ & $\begin{array}{r}0.27559 \\
.66929 \\
1.06299 \\
1.45669 \\
1.85039\end{array}$ & $\begin{array}{r}0.31496 \\
.70866 \\
1.10236 \\
1.49606 \\
1.88976\end{array}$ & $\begin{array}{r}0.35433 \\
.74803 \\
1.14173 \\
1.53543 \\
1.92913\end{array}$ \\
\hline $\begin{array}{l}50 \\
60 \\
70 \\
80 \\
90\end{array}$ & $\begin{array}{l}1.96850 \\
2.36220 \\
2.75590 \\
3.14960 \\
3.54330\end{array}$ & $\begin{array}{l}2.00787 \\
2.40157 \\
2.79527 \\
\text { 3. } 18897 \\
\text { 3. } 58267\end{array}$ & $\begin{array}{l}2.04724 \\
2.44094 \\
2.83464 \\
3.22834 \\
3.62204\end{array}$ & $\begin{array}{l}2.08661 \\
2.48031 \\
2.87401 \\
3.26771 \\
3.66141\end{array}$ & $\begin{array}{l}2.12598 \\
2.51968 \\
2.91338 \\
3.30708 \\
3.70078\end{array}$ & $\begin{array}{l}2.16535 \\
2.55905 \\
2.95275 \\
3.34645 \\
3.74015\end{array}$ & $\begin{array}{l}2.20472 \\
2.59842 \\
2.99212 \\
3.38582 \\
3.77952\end{array}$ & $\begin{array}{l}2.24409 \\
2.63779 \\
3.03149 \\
3.42519 \\
3.81889\end{array}$ & $\begin{array}{l}2.28346 \\
2.67716 \\
3.07086 \\
3.46456 \\
3.85826\end{array}$ & $\begin{array}{l}\text { 2. } 32283 \\
\text { 2. } 71653 \\
\text { 3. } 11023 \\
\text { 3. } 50393 \\
\text { 3. } 89763\end{array}$ \\
\hline
\end{tabular}




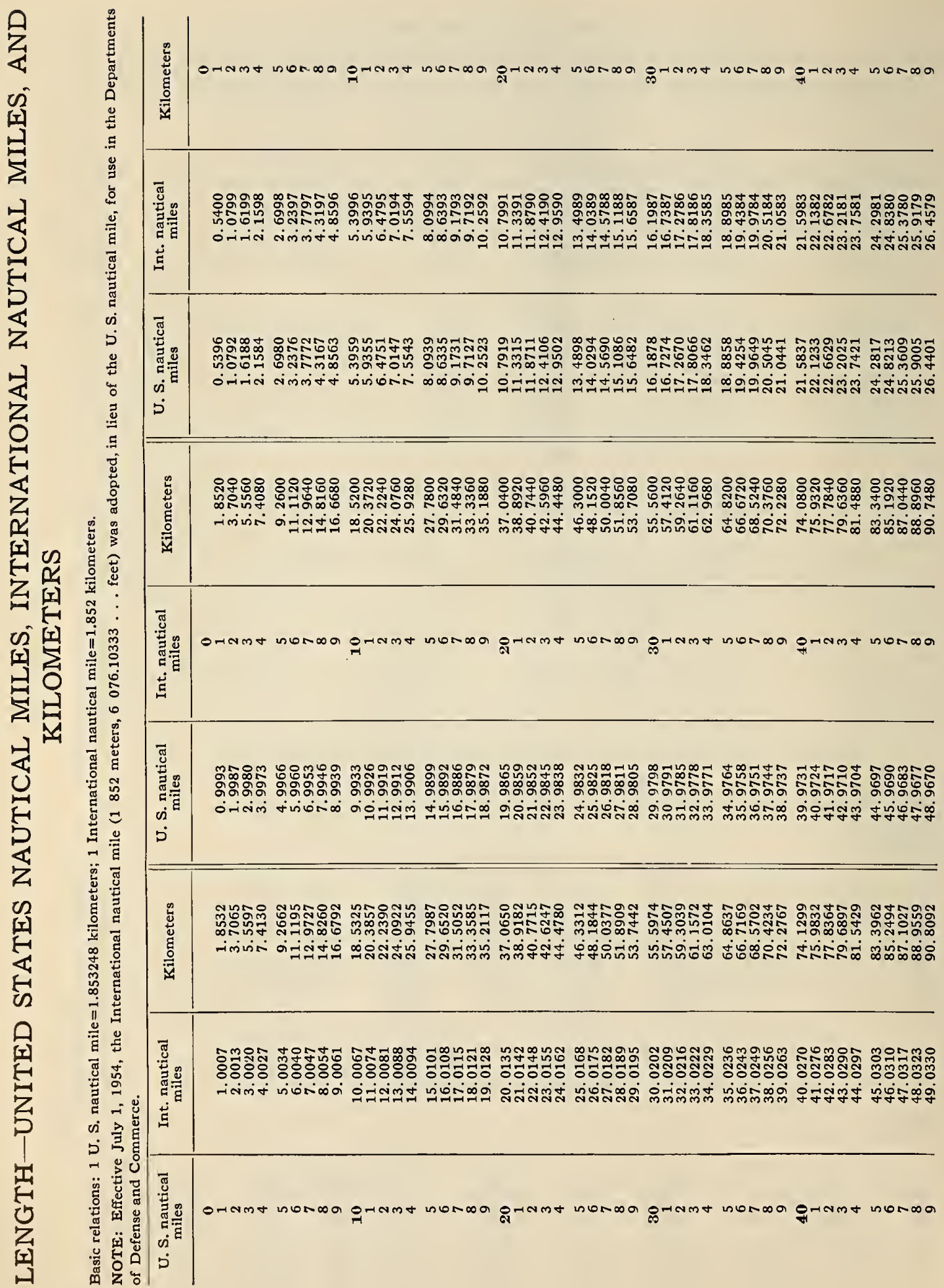




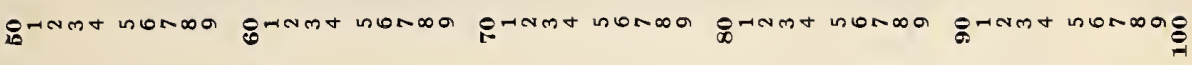

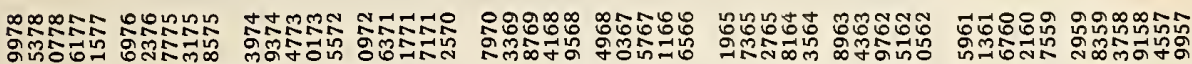

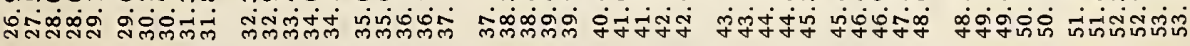

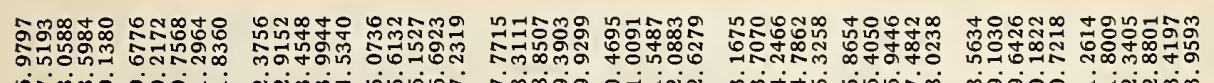

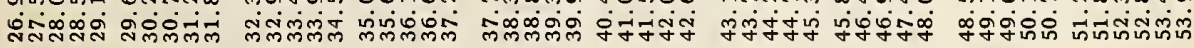

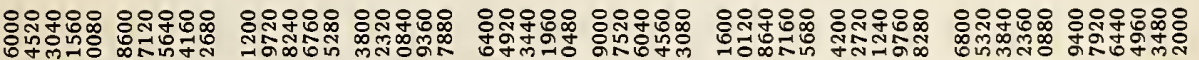

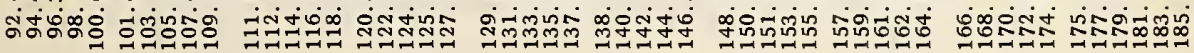

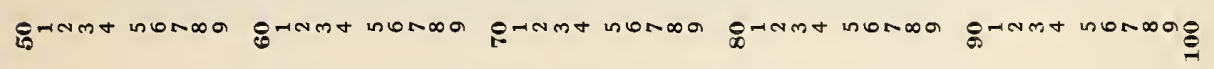

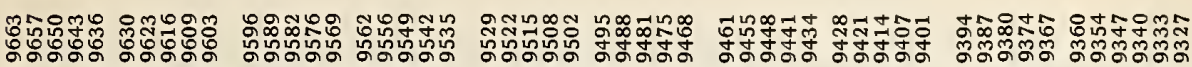

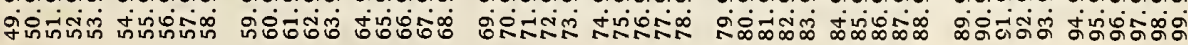

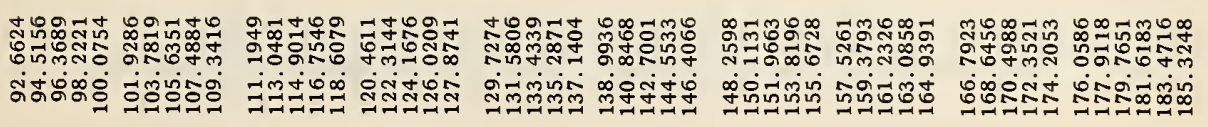

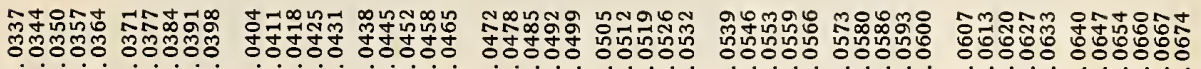

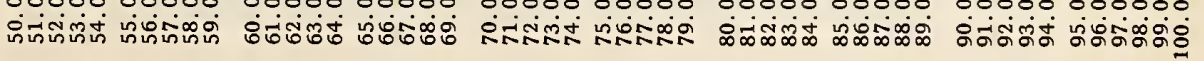

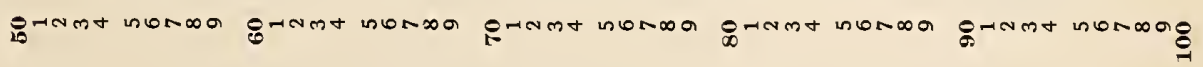




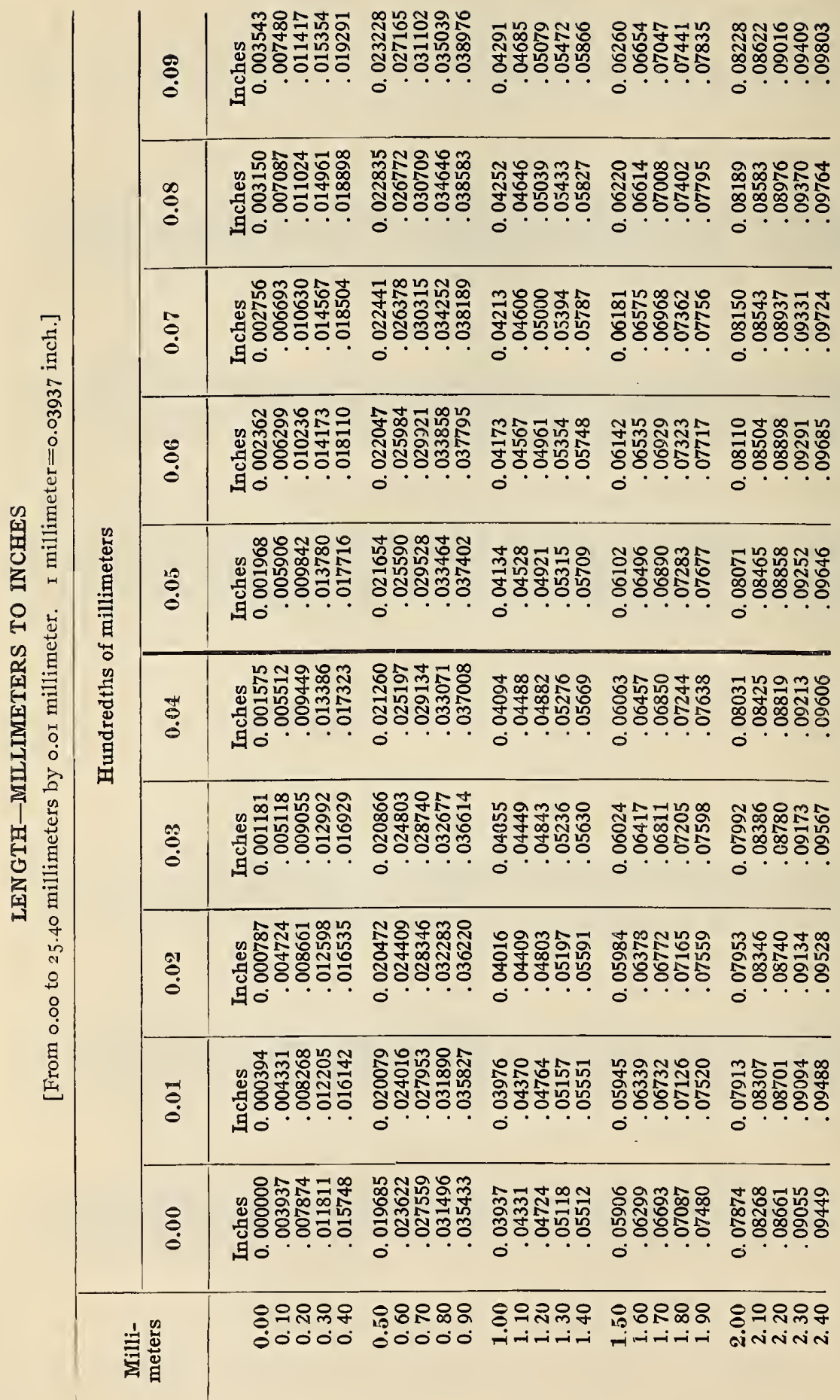




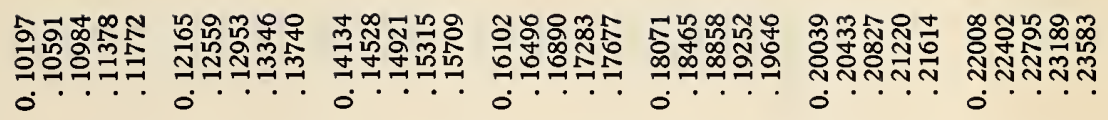

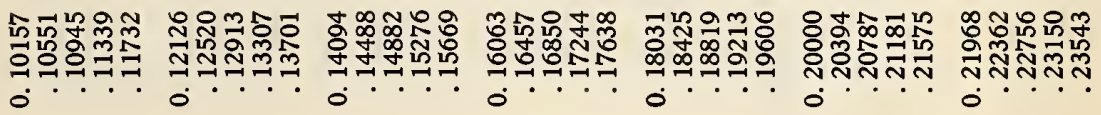

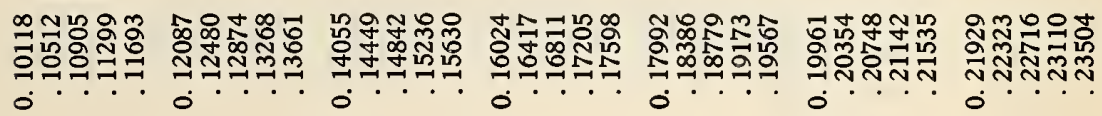

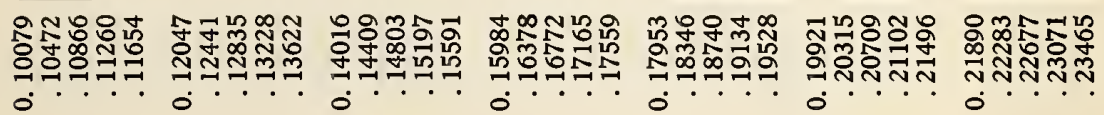

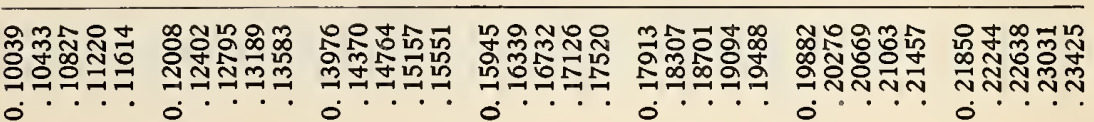

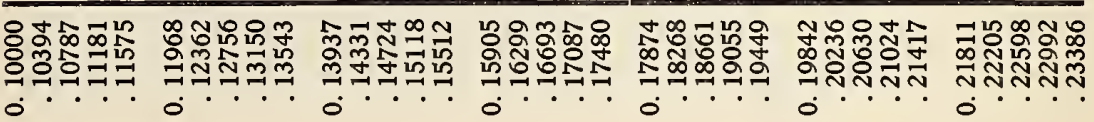

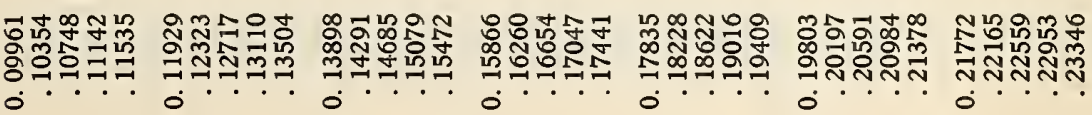

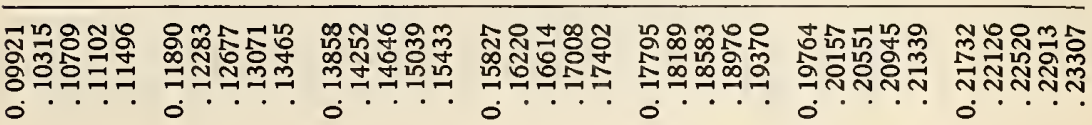

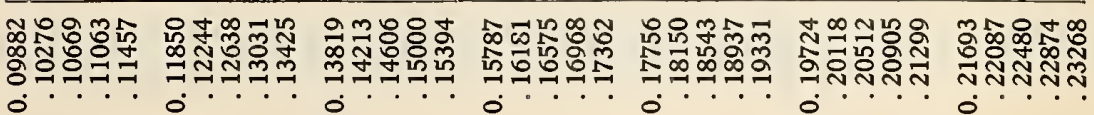

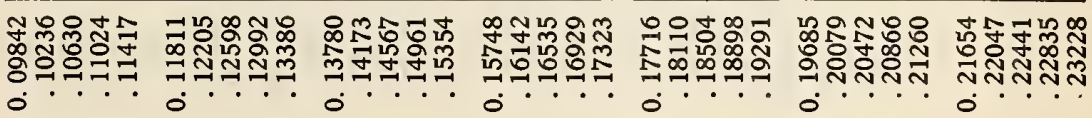

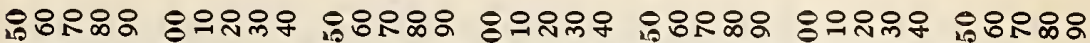

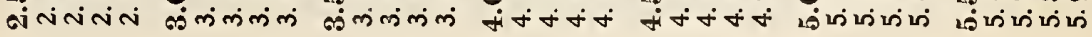




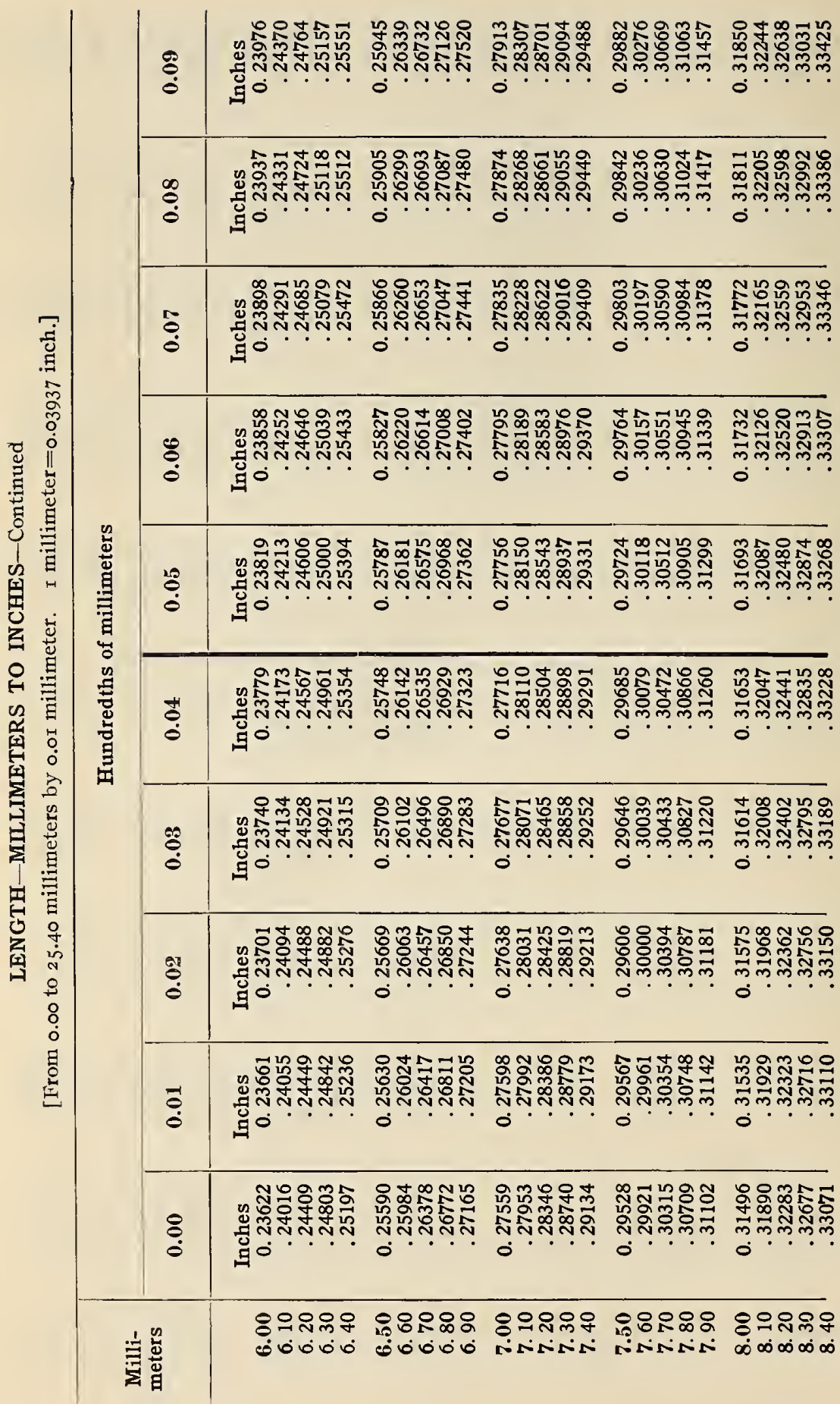




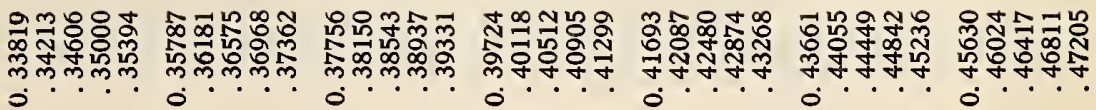

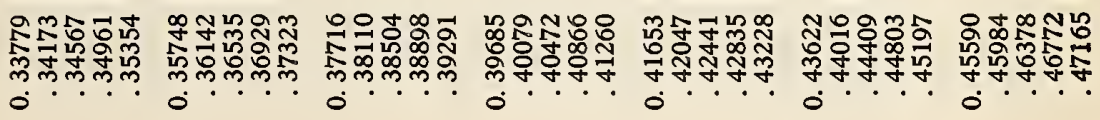

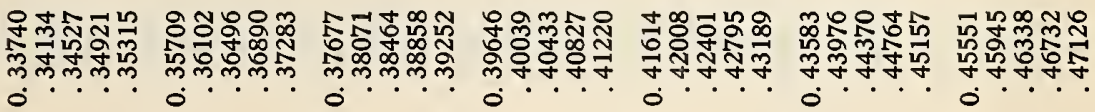

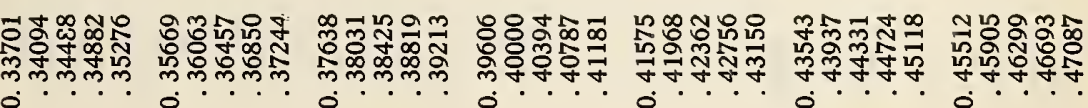

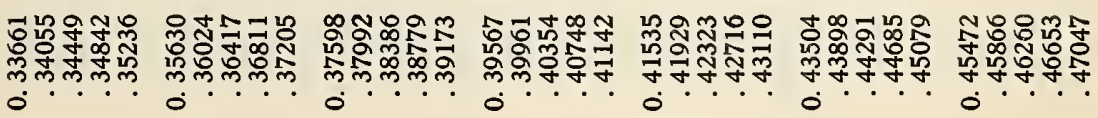

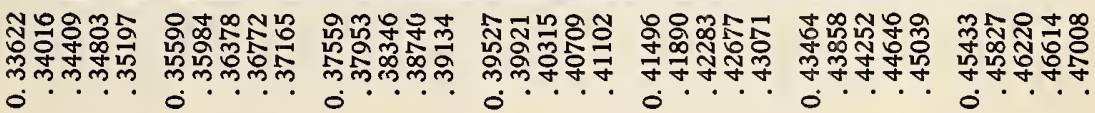

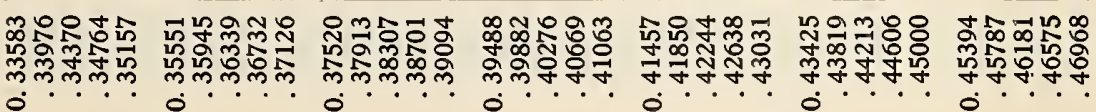

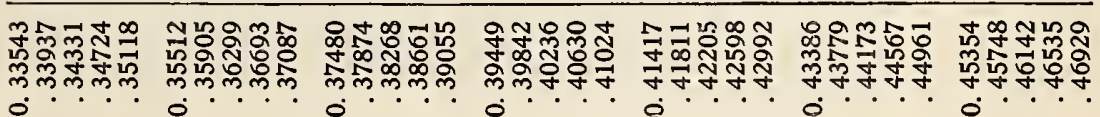

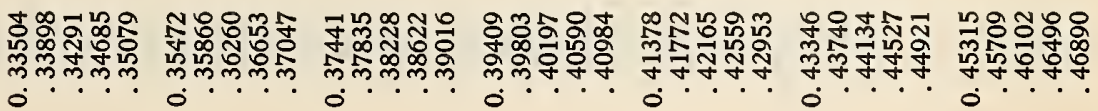

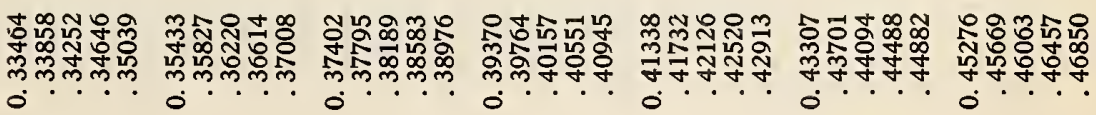

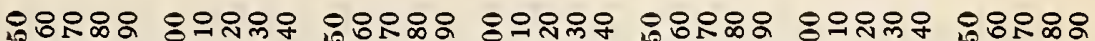

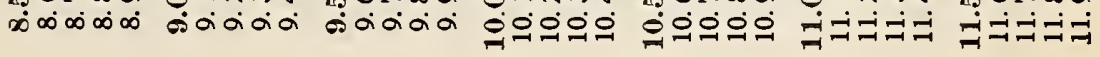




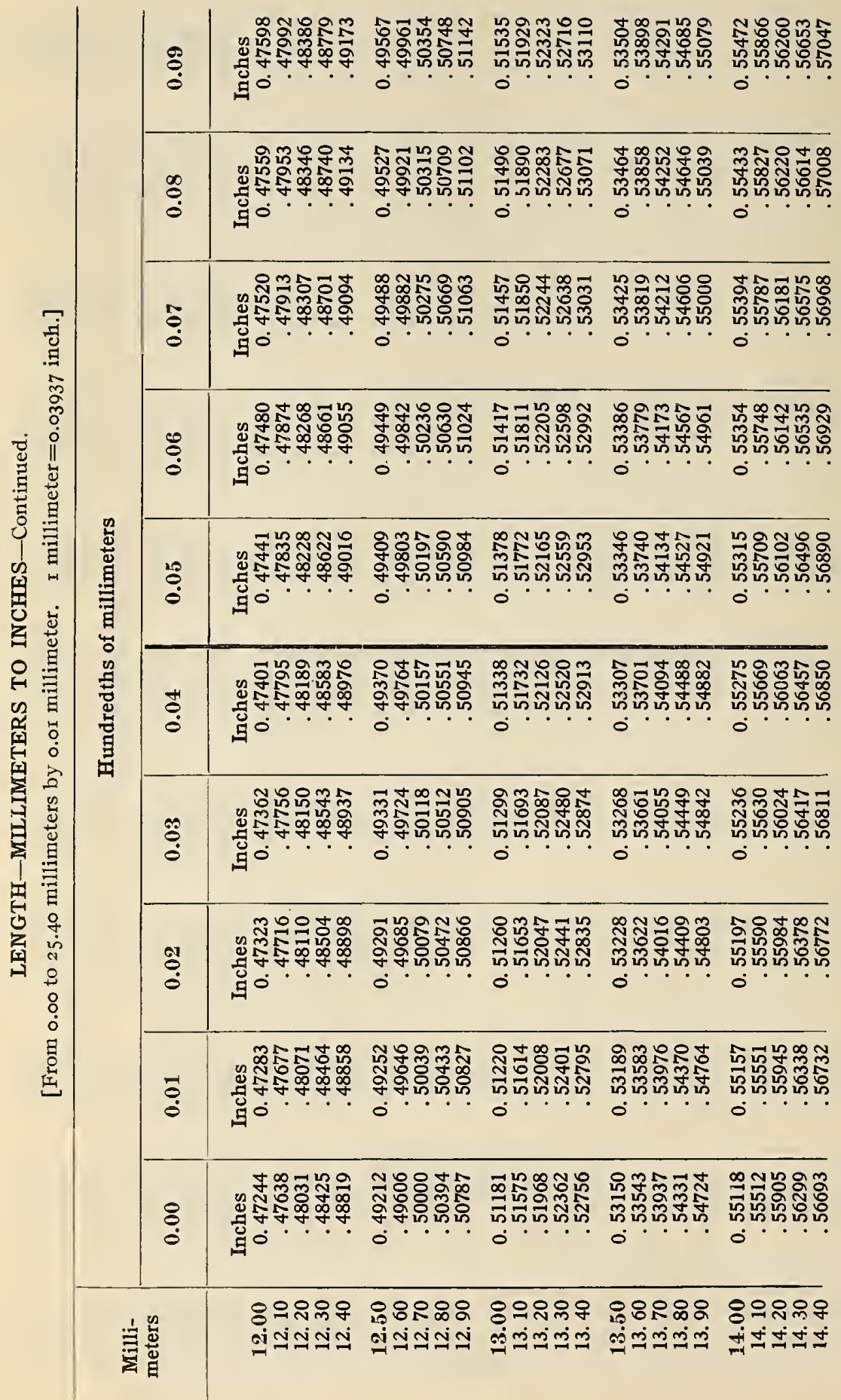




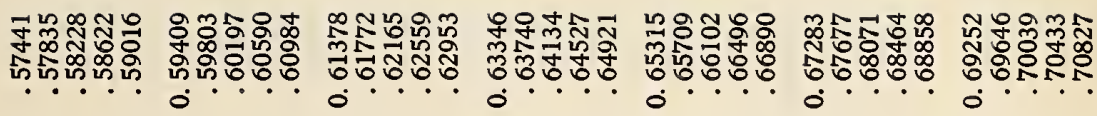

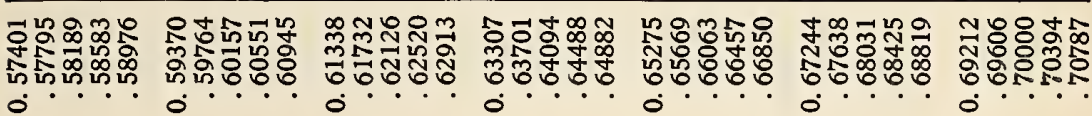

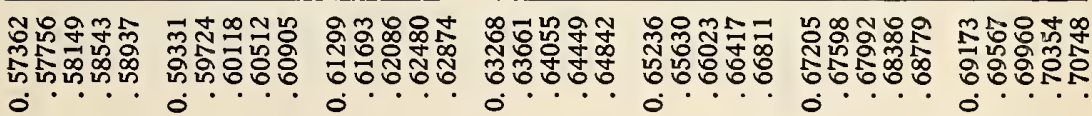

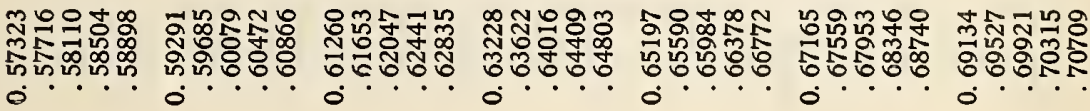

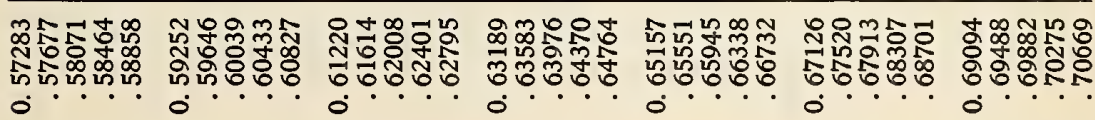

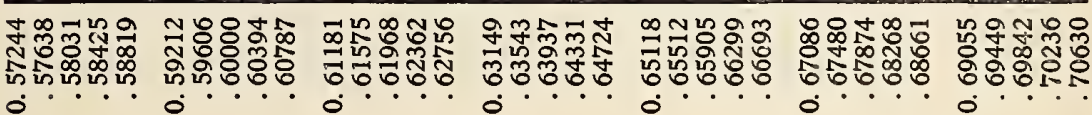

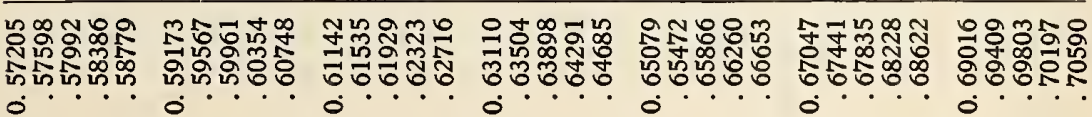

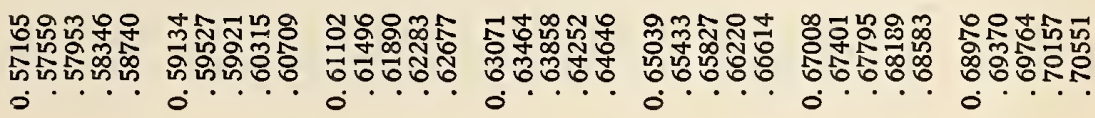

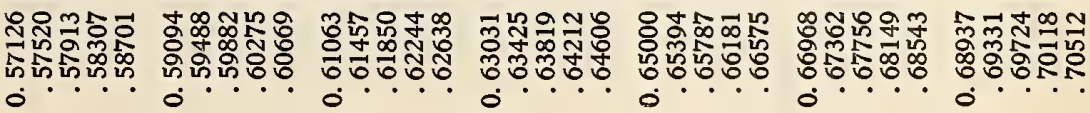

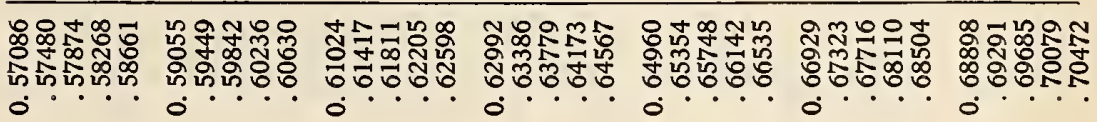

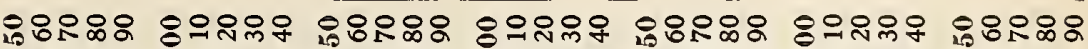

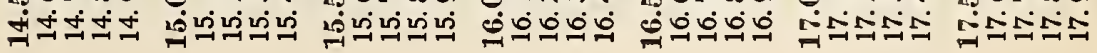




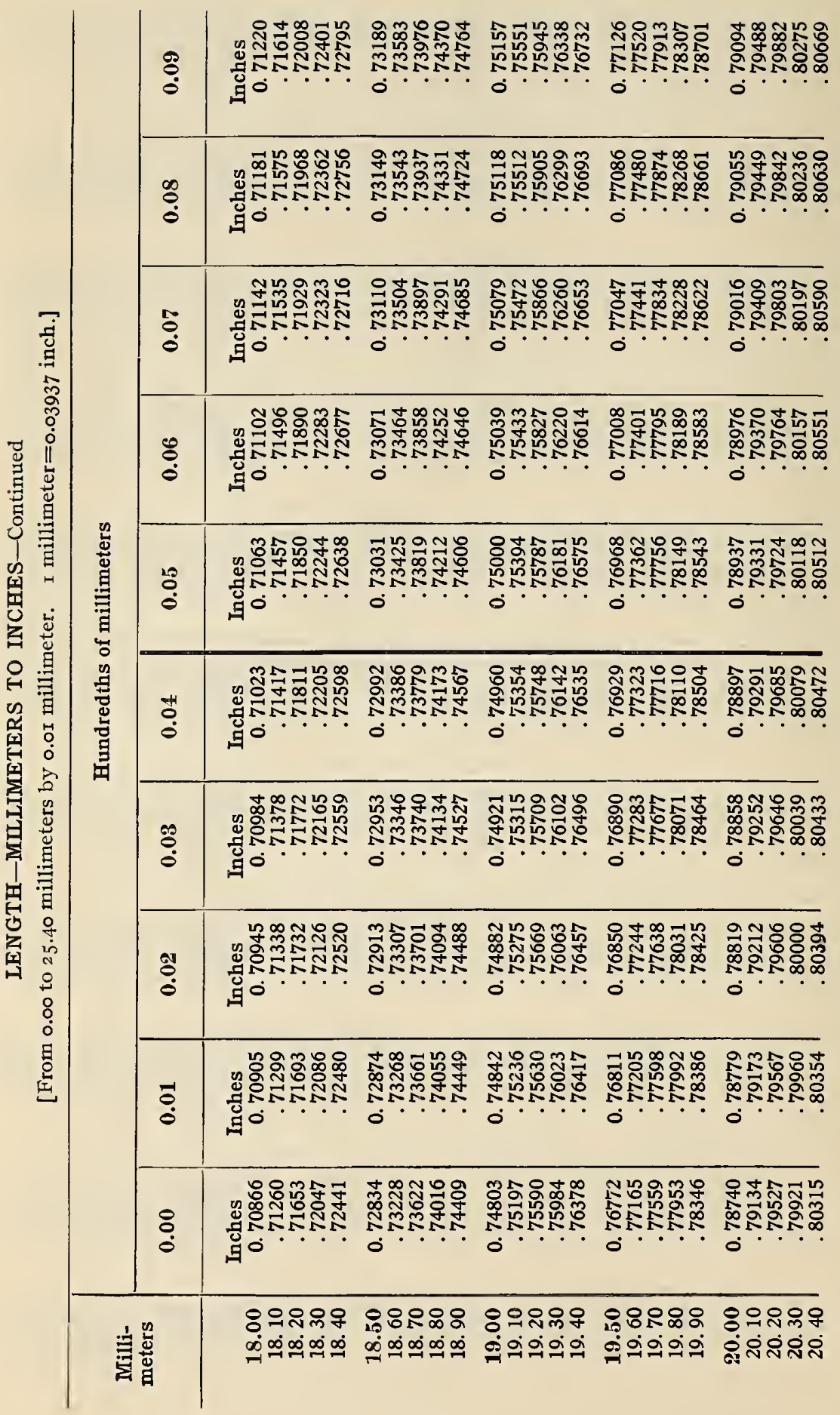



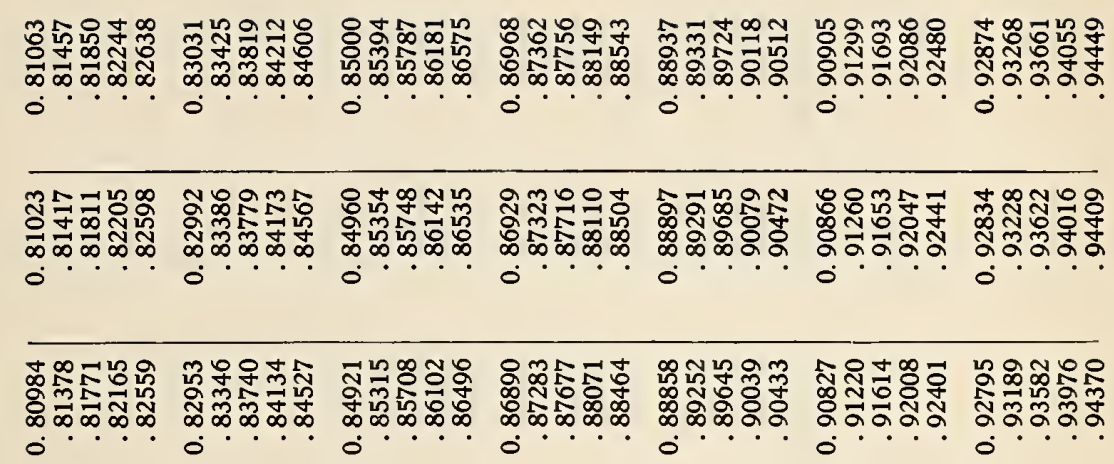

\begin{tabular}{|c|c|c|c|c|c|c|}
\hline 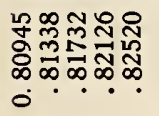 & 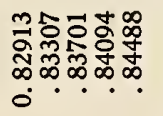 & 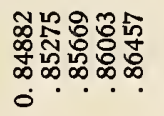 & 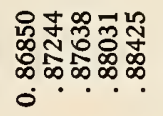 & $\begin{array}{l}\text { 워용요용 } \\
\infty \\
\infty \\
\infty \\
\infty\end{array}$ & 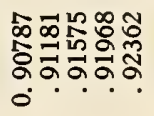 & 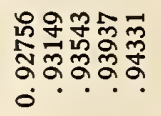 \\
\hline
\end{tabular}

\begin{tabular}{|c|c|c|c|c|c|c|}
\hline 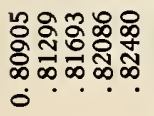 & 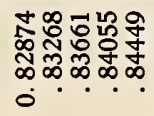 & 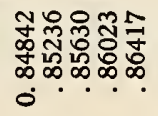 & 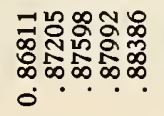 & 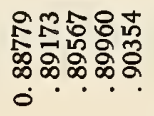 & 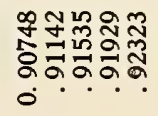 & $\begin{array}{l}\mathbb{N} \\
\text { ๙ू. } \\
\dot{0}\end{array}$ \\
\hline
\end{tabular}

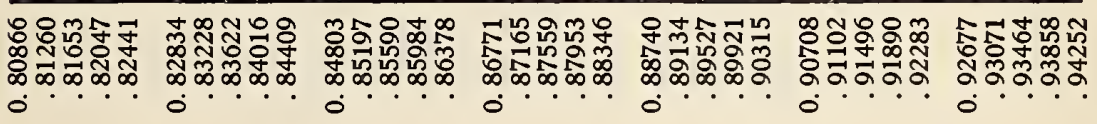

\begin{tabular}{|c|c|c|c|c|c|c|}
\hline 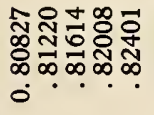 & 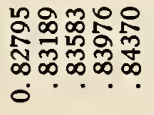 & 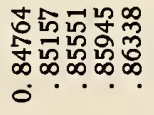 & 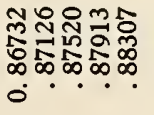 & 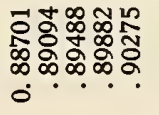 & 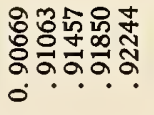 & 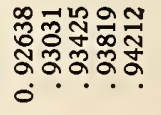 \\
\hline
\end{tabular}

\begin{tabular}{|c|c|c|c|c|c|c|}
\hline 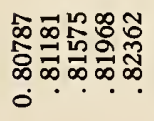 & 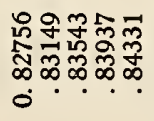 & 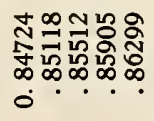 & 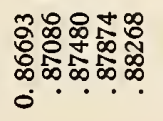 & 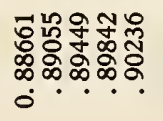 & 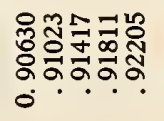 & 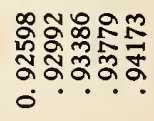 \\
\hline
\end{tabular}

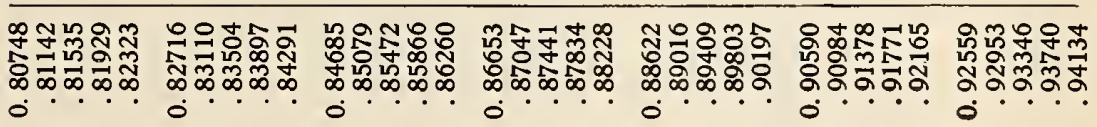

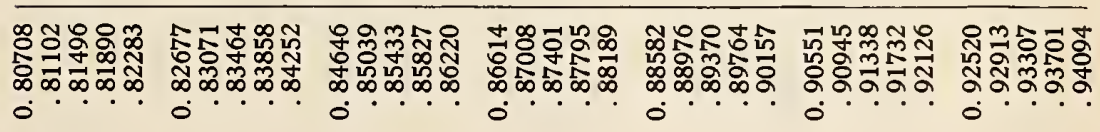

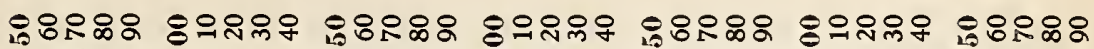

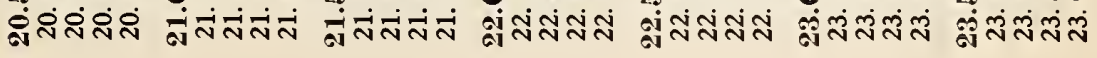




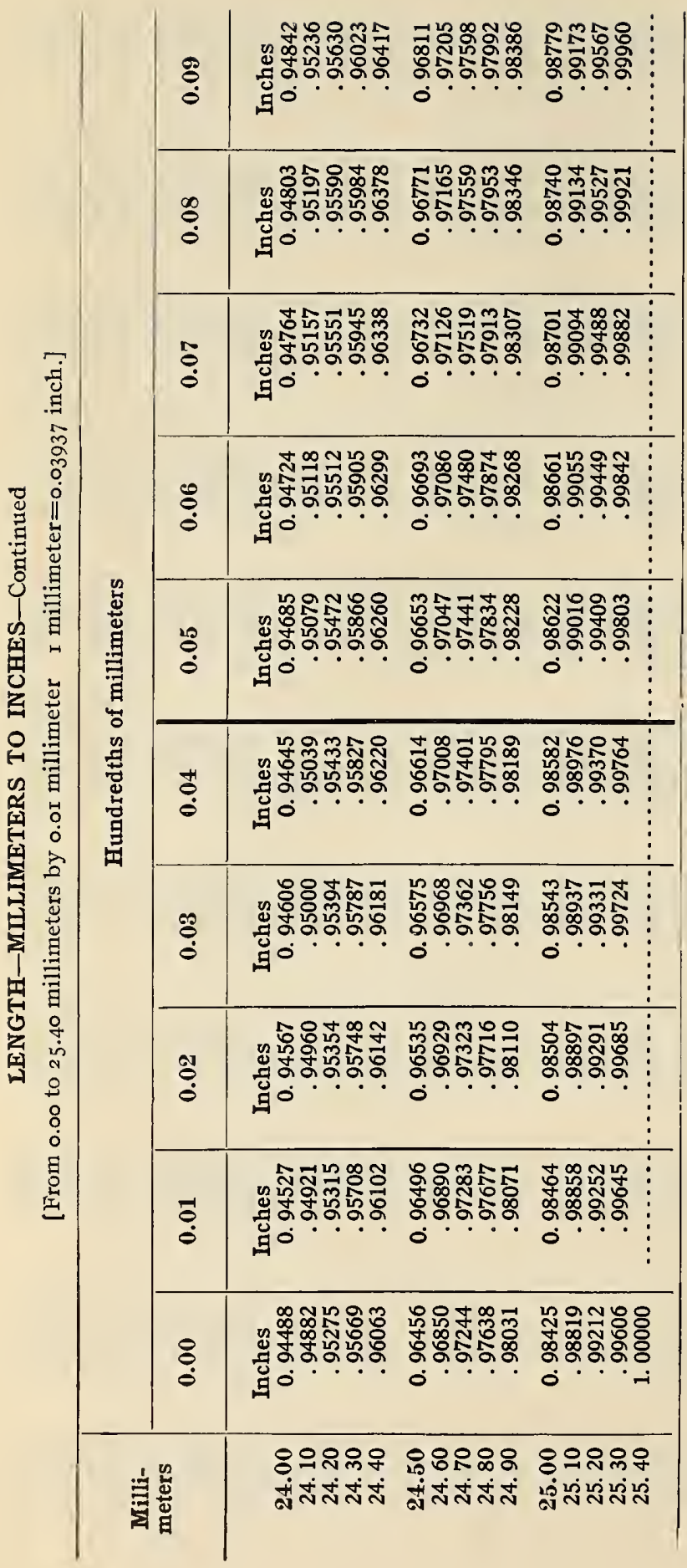


9. TABLES OF EQUIVALENTS FROM 1 TO 999 UNITS

27 


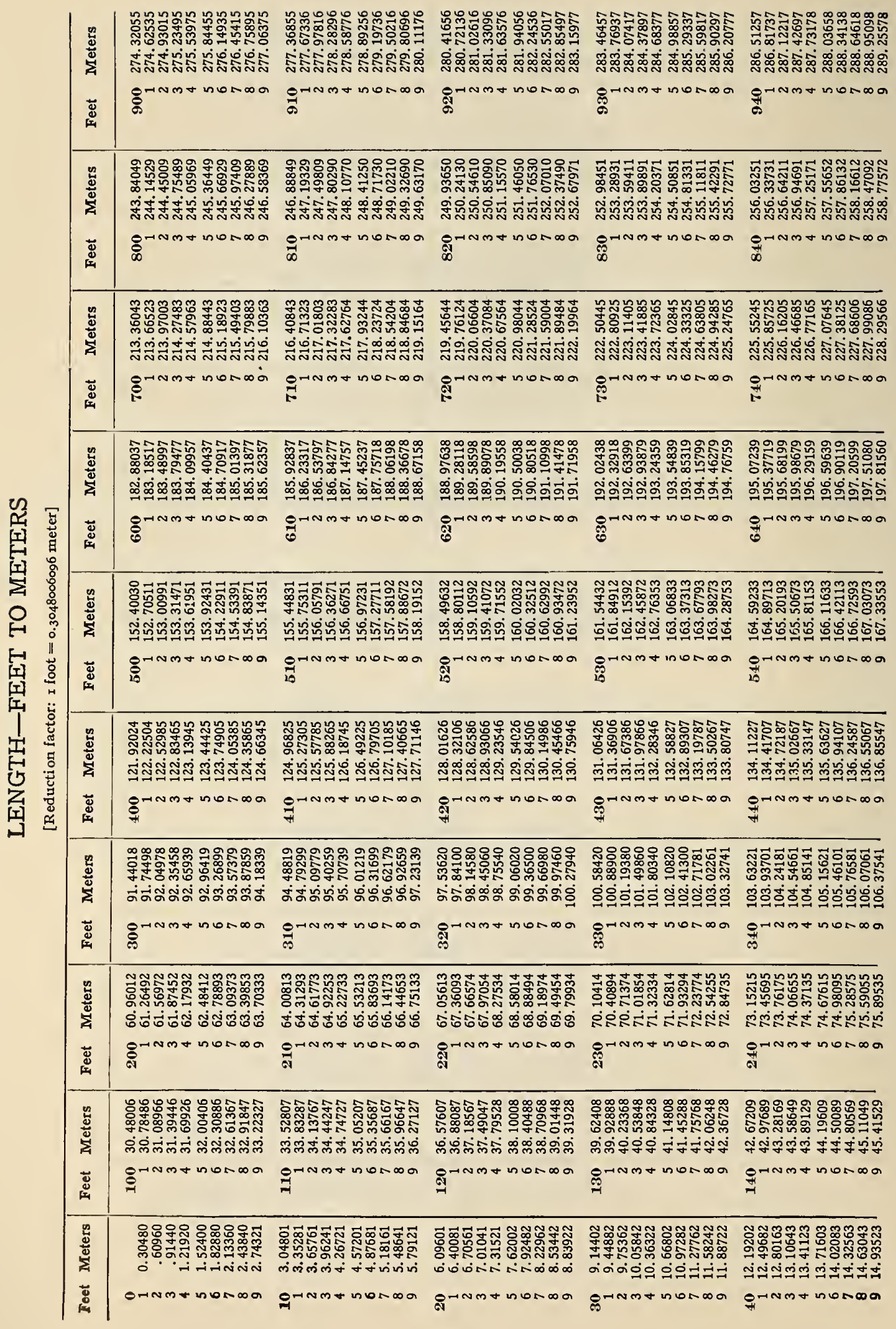




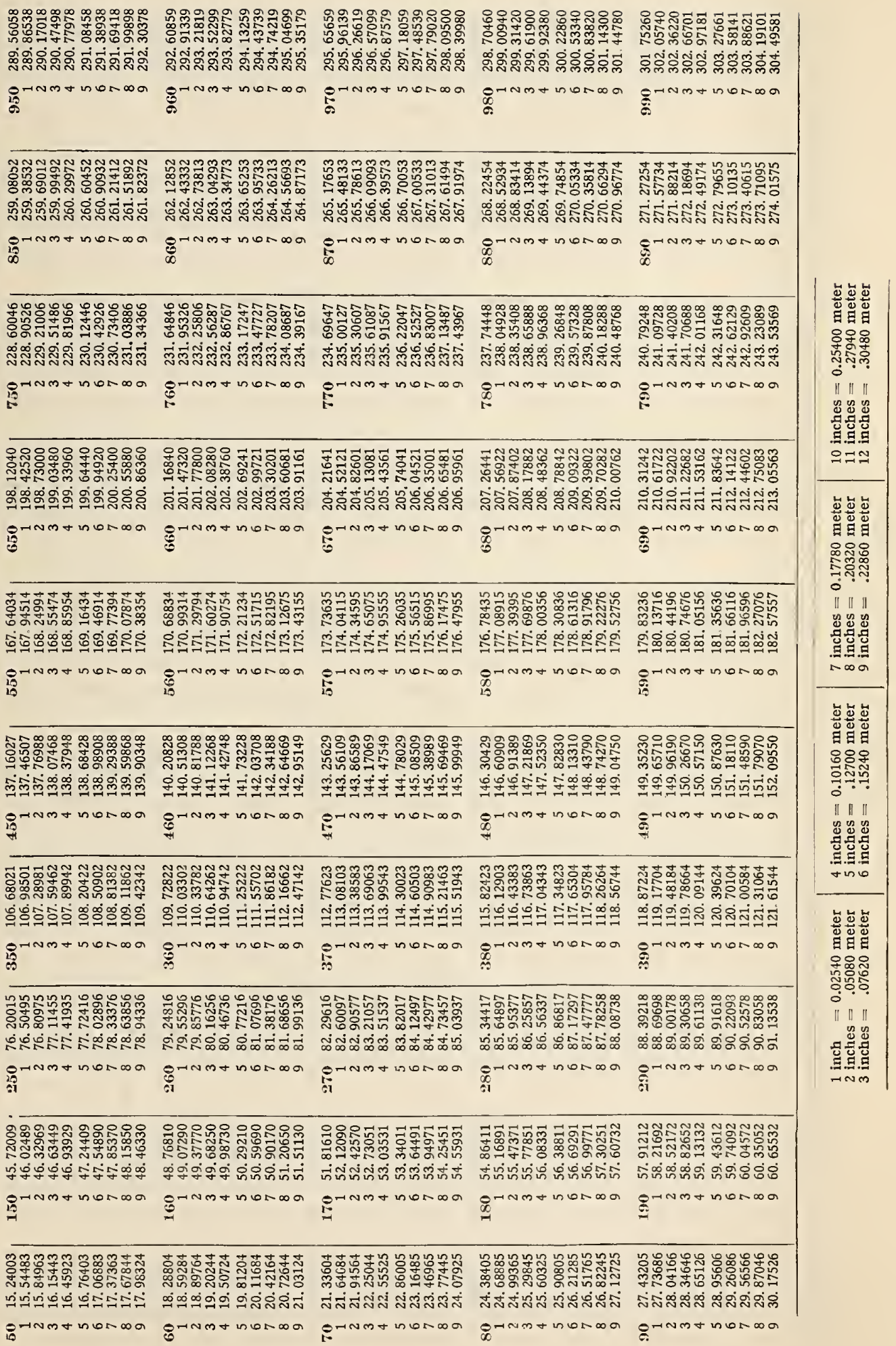




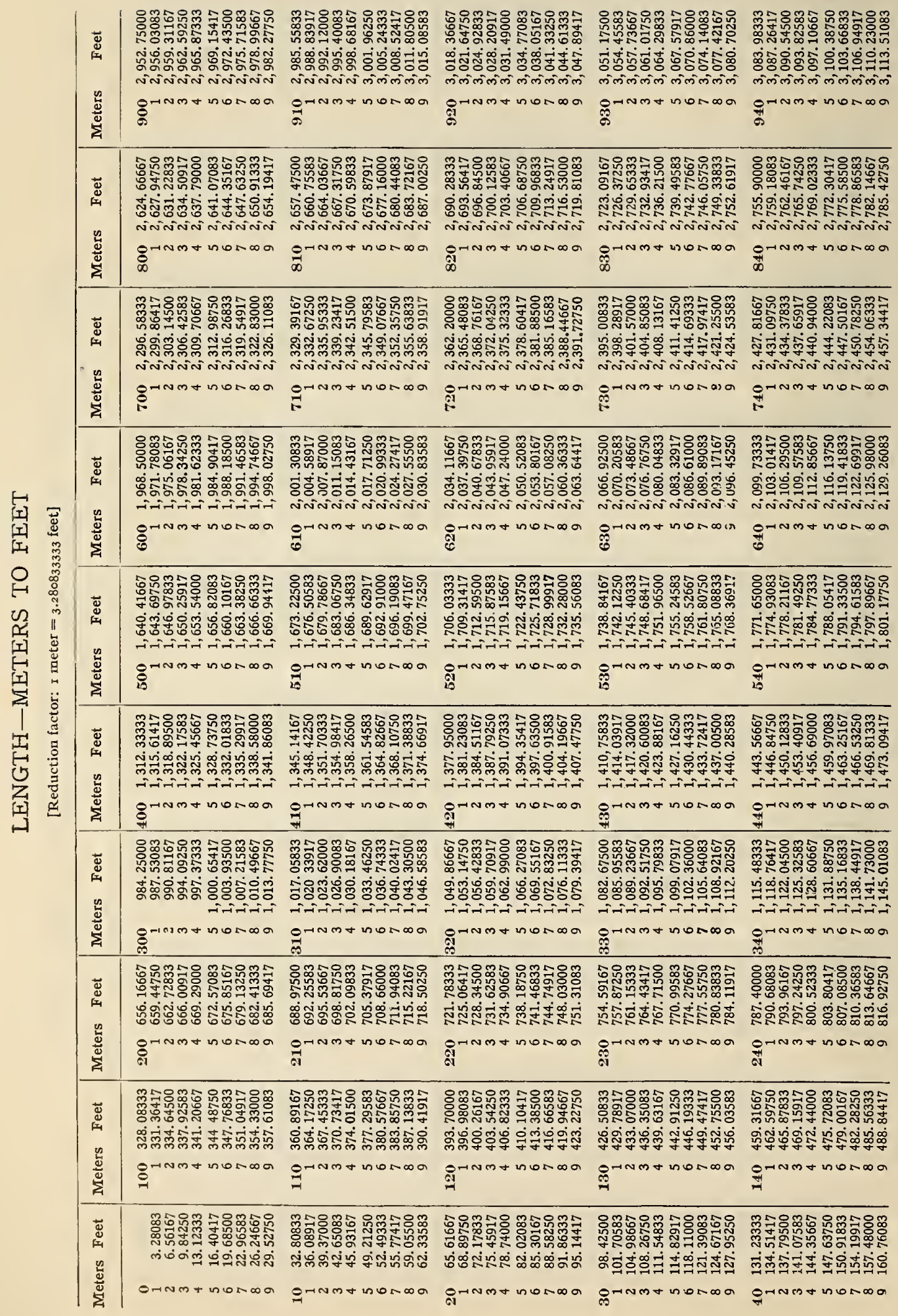




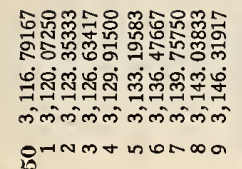

है

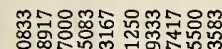
엉새

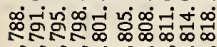

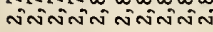

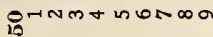
$\infty$

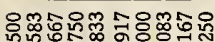

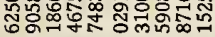

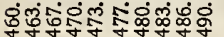
Nivinasinivicis PrNm+nondo is

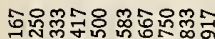

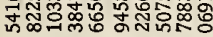

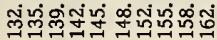

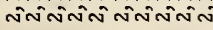
Brnmanona

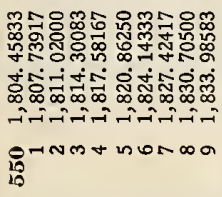

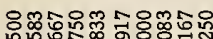

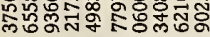

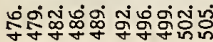

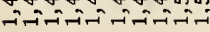
OHNmanoma

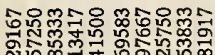

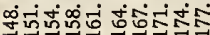

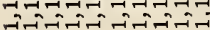
OHNMtUONDa in

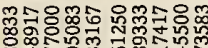

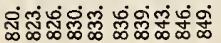
量

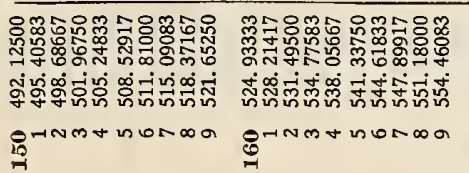

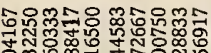

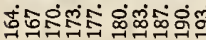

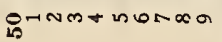

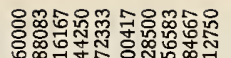

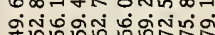
$\Rightarrow=-1=1$

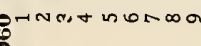

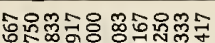

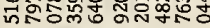

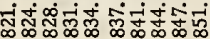
NंNiNiNNTNiN 8

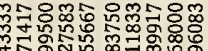
ஆัgं் NANivivivives Ernmanora \&

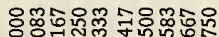

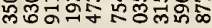

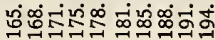
NiniviñNiñNín 8

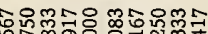

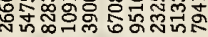

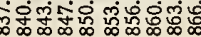

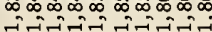
ofnmanona

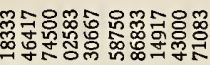

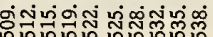

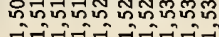

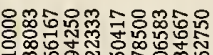

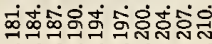

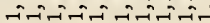
PrNm+nONo

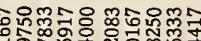

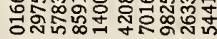

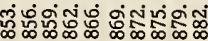
OrNman nondo

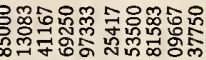

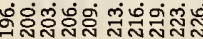
8rnotinuma ErNmanuma

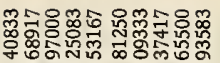

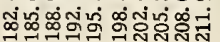

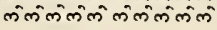
है

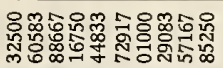

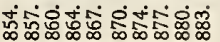
जivivin Nivinis PrNmtயONDo

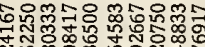
oinio rivion

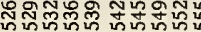

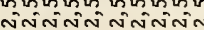

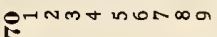
F

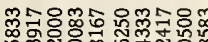

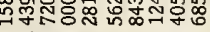

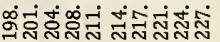
inivinivinivision 是

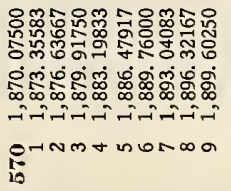

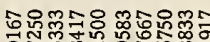

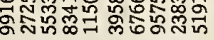

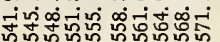

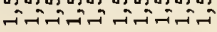
Etnmanana

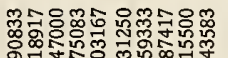

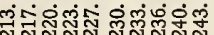

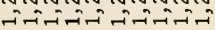
PrNmanna

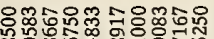

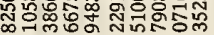
ฒં ernmanoma

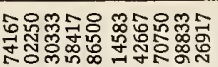

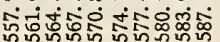
PrNmanona

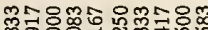

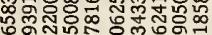

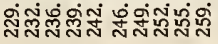

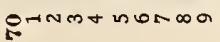

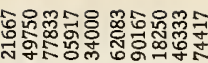
దే नंगुलन

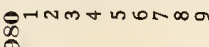

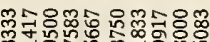

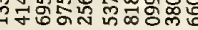

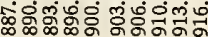

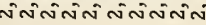
$\infty_{\infty}^{\infty}$

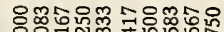

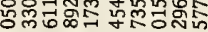

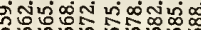

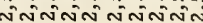
OrNm+nONo

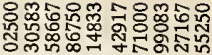

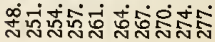

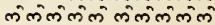
PrNm+nona

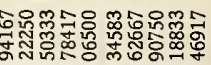

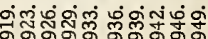

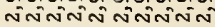
8-nmonणma

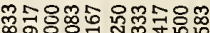

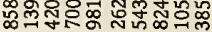

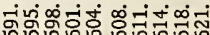

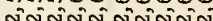
ofnmanoma

ㅎํㅇํำ

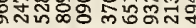

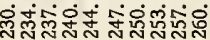

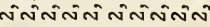

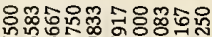

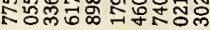

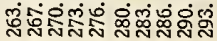

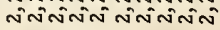
옹

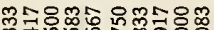

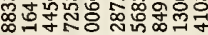

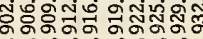

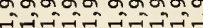
Prnmonoma

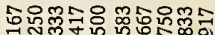

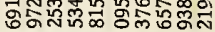

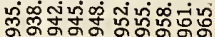
जनiनت OrNmanuma

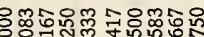

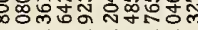

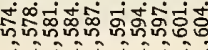

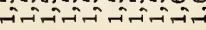

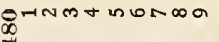

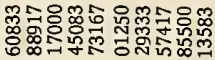

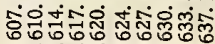

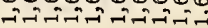
PrNm numa

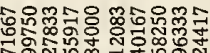

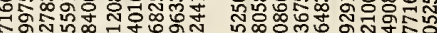

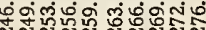

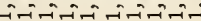

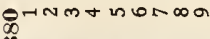

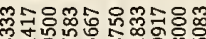

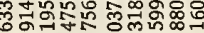

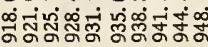

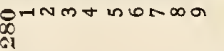

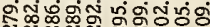

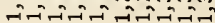
OrNmanuma

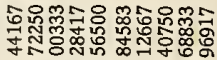

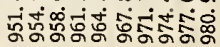
के

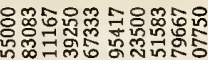

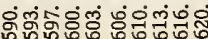
PrNmanona

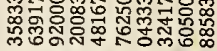

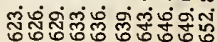
\&+Nm+noma

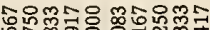

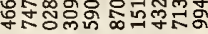

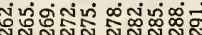

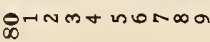

ㅇํํㅇํำ N

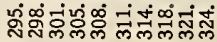
8 


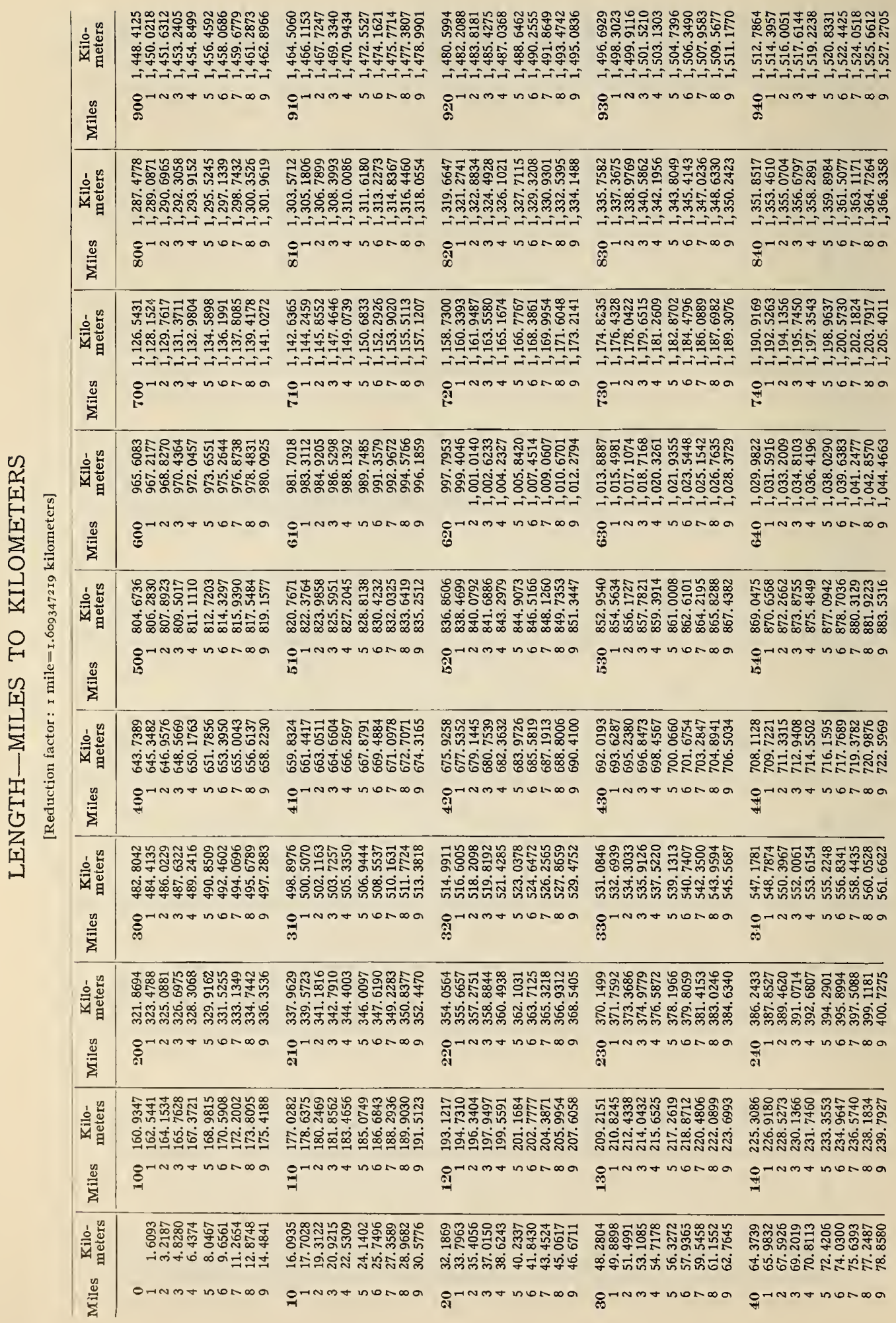




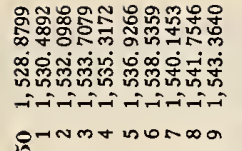

เ

(1)

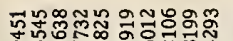
co-

rifint 要

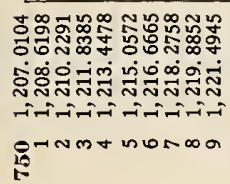

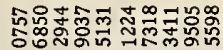

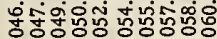

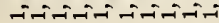
: Grumanoma

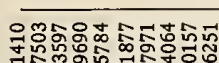

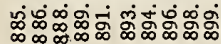

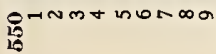

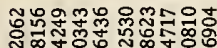

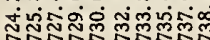

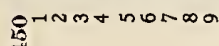

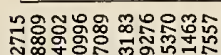

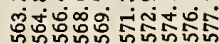

잃

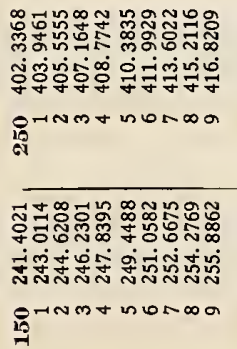

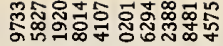

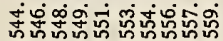

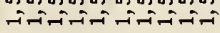
8

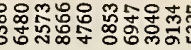

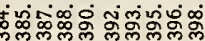
initifi-iti ginetruma

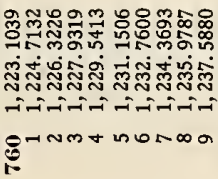

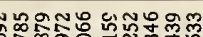
승ㅇㅇ

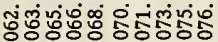

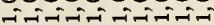
\&inmanorna

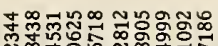

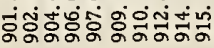
४्व :

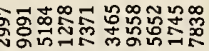

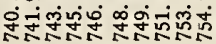
ofunturanos

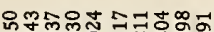

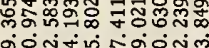

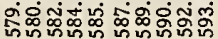
\%

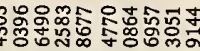

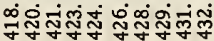

formotionoma

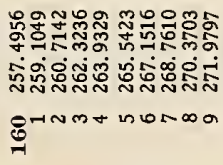

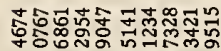

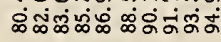
8

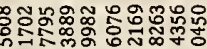

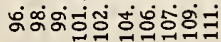
8

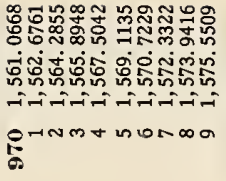

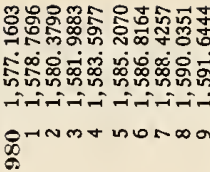

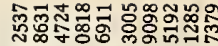
:

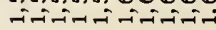
ornmonomo

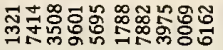

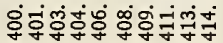

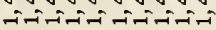
我 ernmonoma

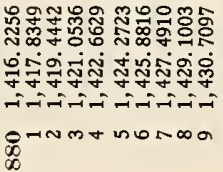

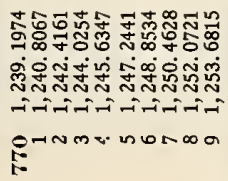

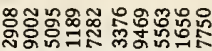

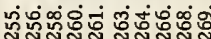

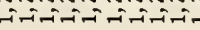
$\underset{1}{\stackrel{D}{1}}$

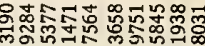

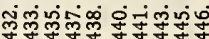

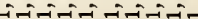
Encutanonom

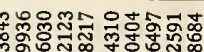

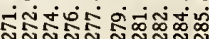

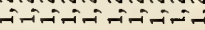

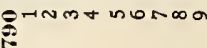

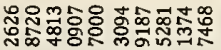

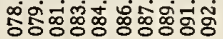

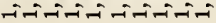
8̈rnotinoma

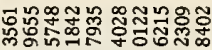

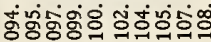

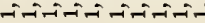
Önmanuma

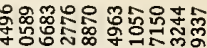

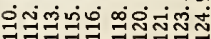

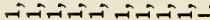
grnmtinona

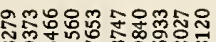

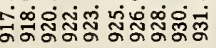
Prinmanomona

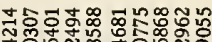

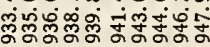

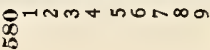

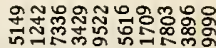

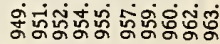
OPrnmanonom

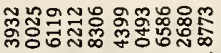

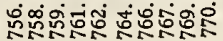
ginmanoma

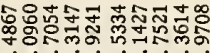

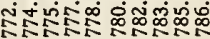
OHNm+ nuR a

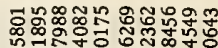

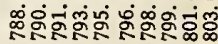

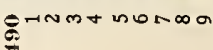

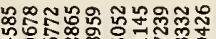

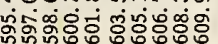

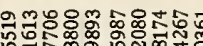

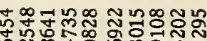

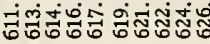

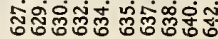
encumanomo 要

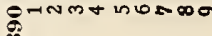

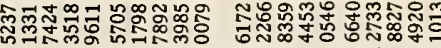

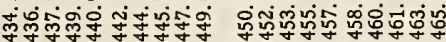
prnmanomana $\infty$
0
0

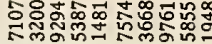

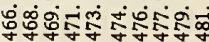
ofrnominoma

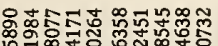
空 Sinmanona

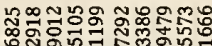

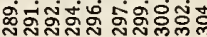
Ornmanuma

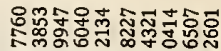

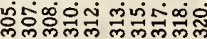
g-nmtnunoma

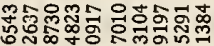
걱

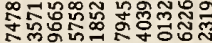

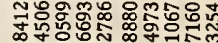

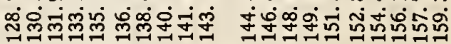
OHNm+norno $\infty$

\section{-}

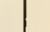




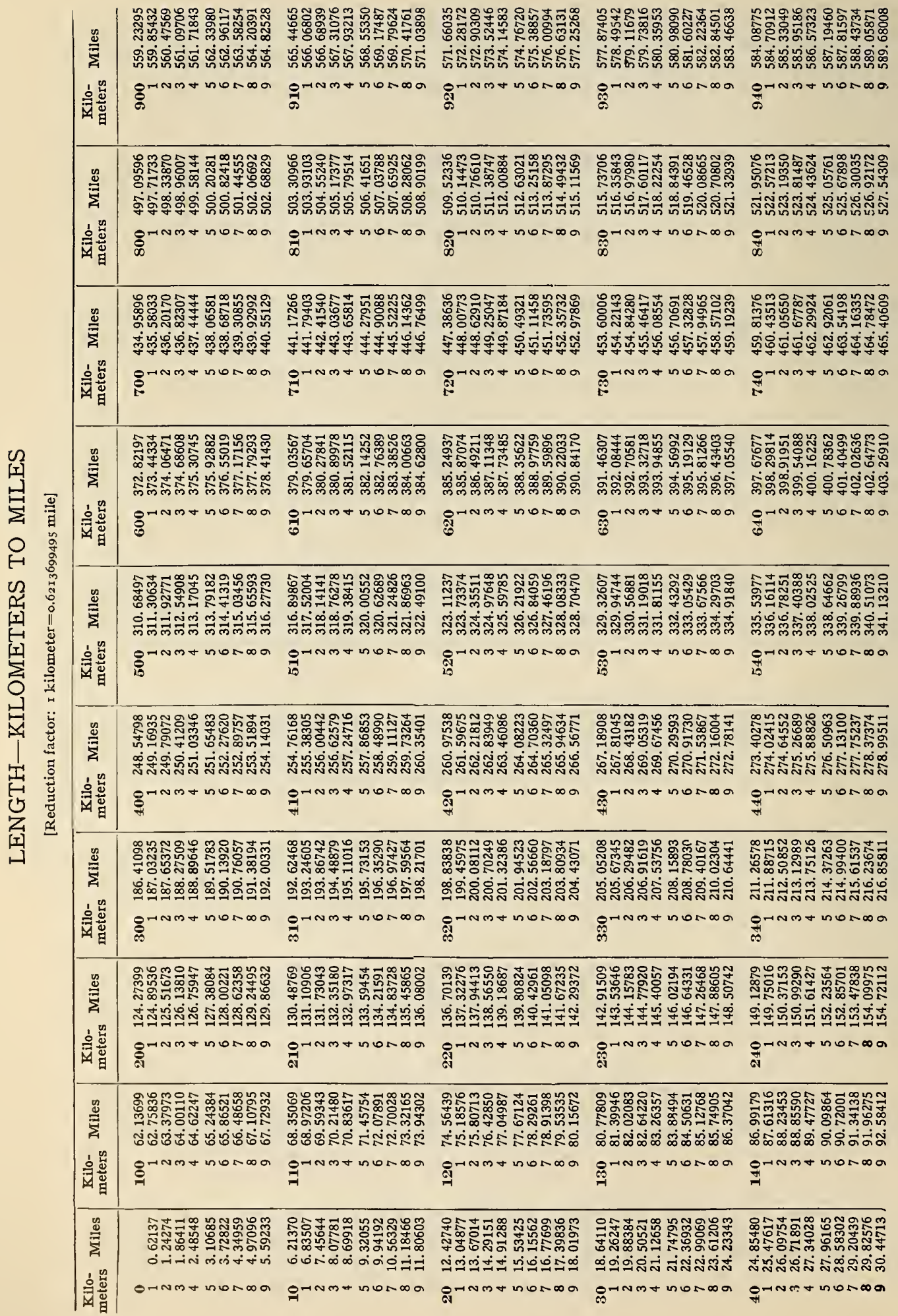



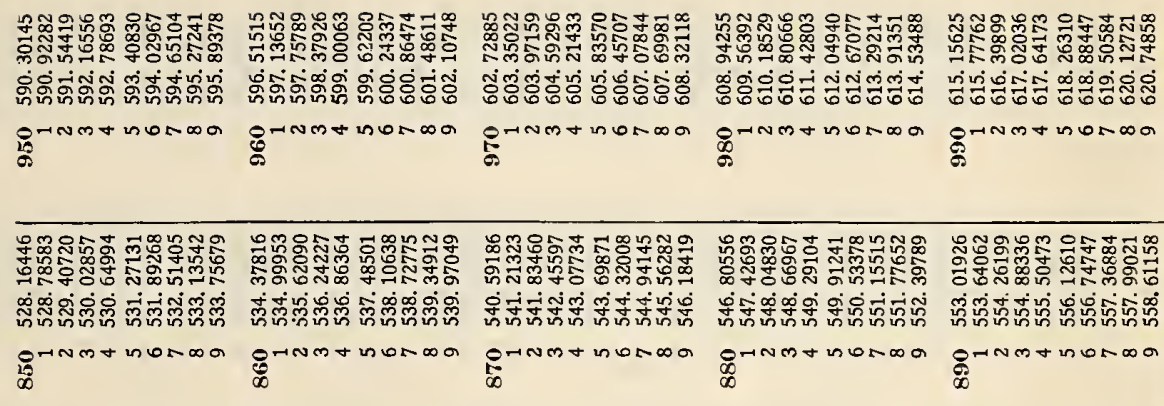

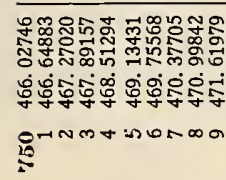

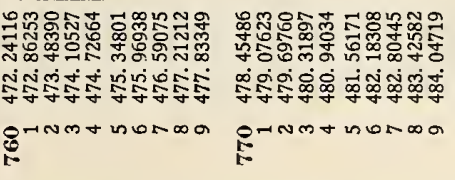

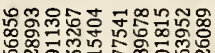

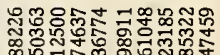

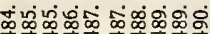

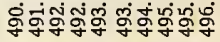

D-nm+nuna क्षintnonos

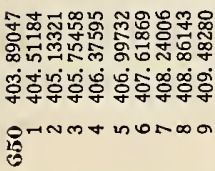

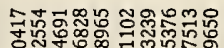

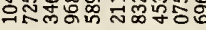

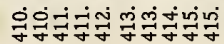

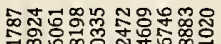

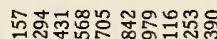

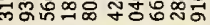

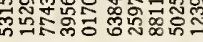
8rinmanuma

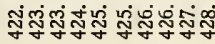

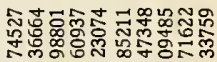

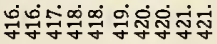
Prnmanoma

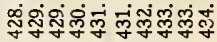

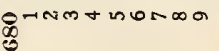
Prnmanona

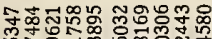

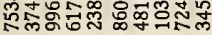

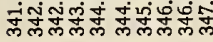

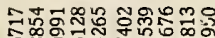

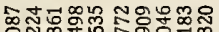

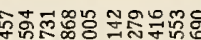

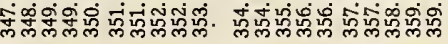

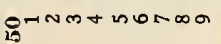
8 PrNmanona ○ంல :

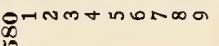

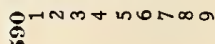

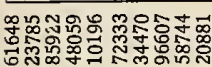

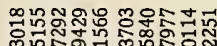

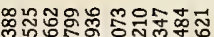

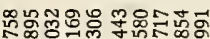

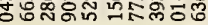

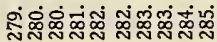

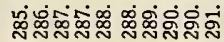

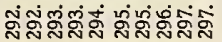
更

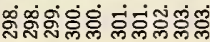

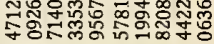
Prumanomo Brnmanoma

EnNmanoma

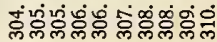

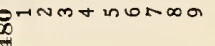

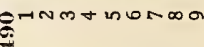

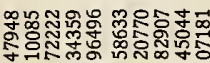

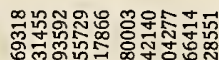

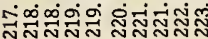

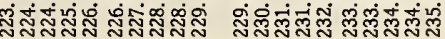
㩆

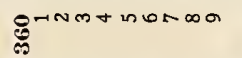
grnmantina

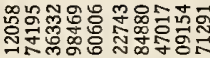

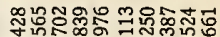

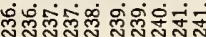

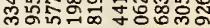

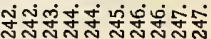

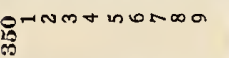

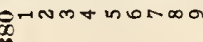
grnmantina

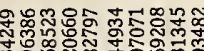

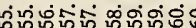

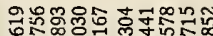

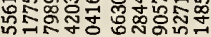

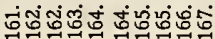

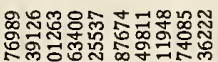

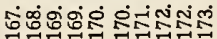

orrumanuma grnmanuman

甼

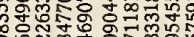

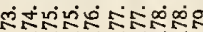

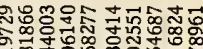

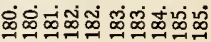
ติ बै 赵

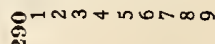

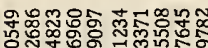

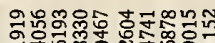

영용ำ

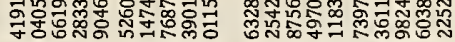

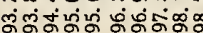

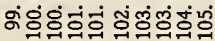

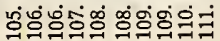

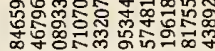

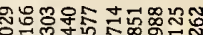
o시의

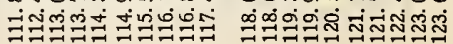

PINm+nטNon

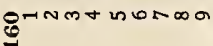

PrNmanono $\underset{\sim}{\infty}$

8

애웜유.

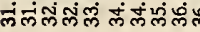

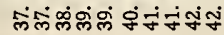

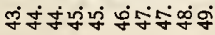

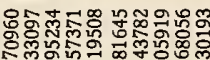

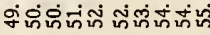

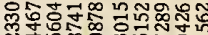
8rnmanoma 앙 $\infty$

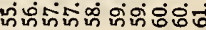
8 grnmanoma PHNm+norma 8 


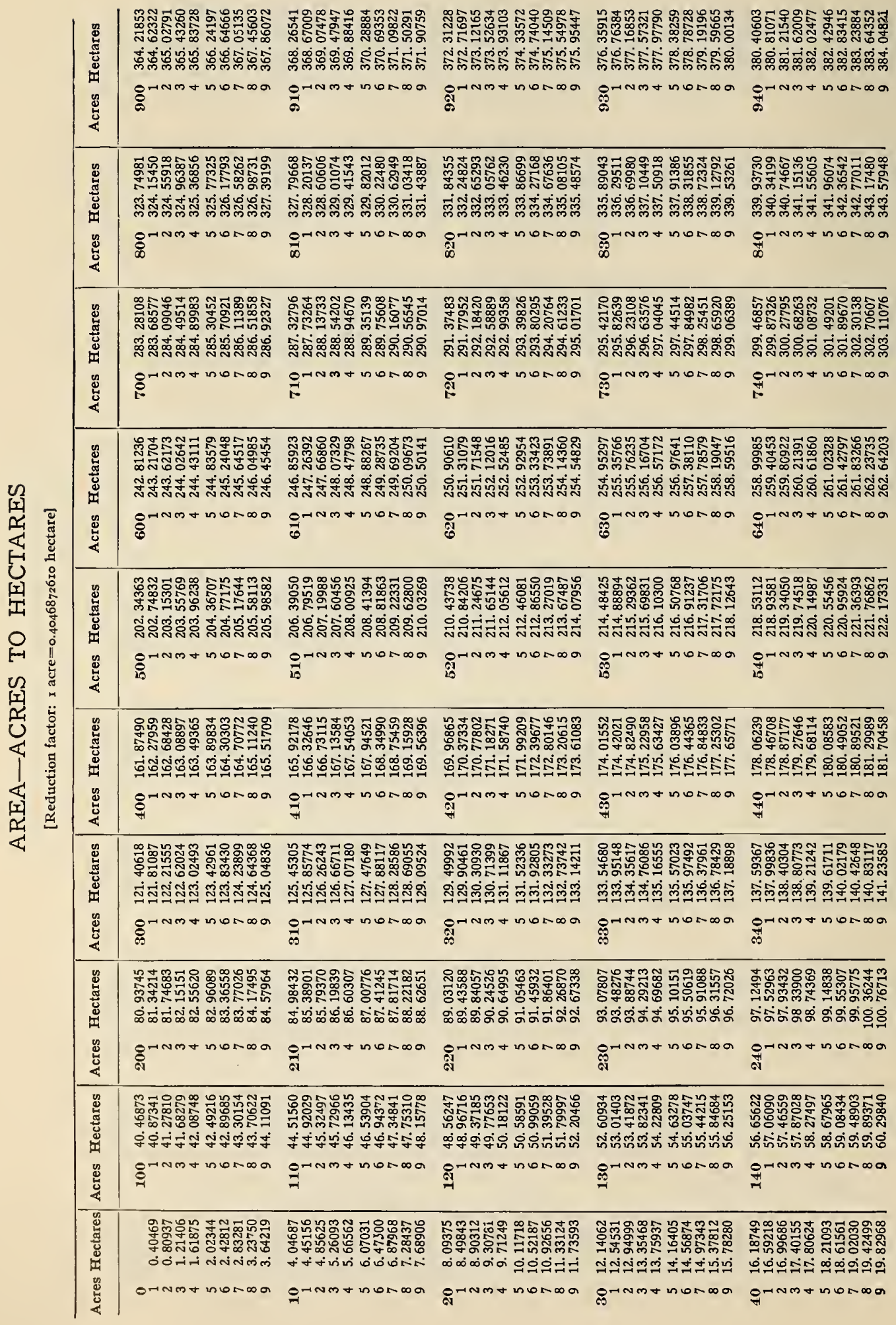




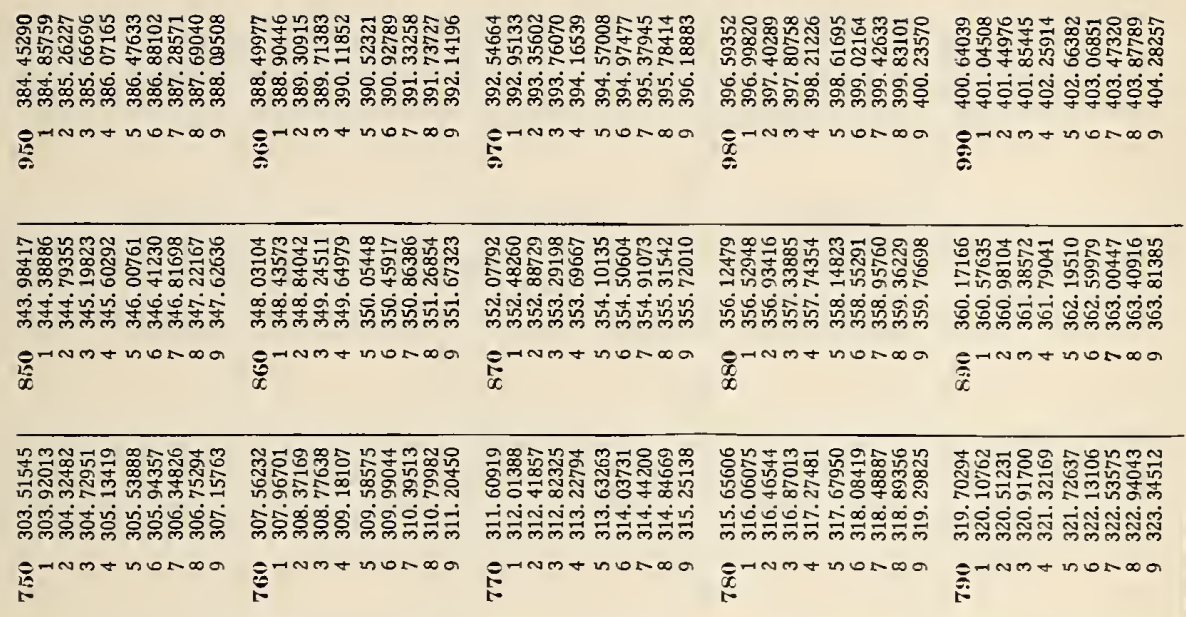

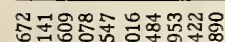

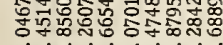

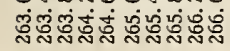

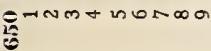

\%

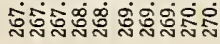
है

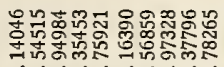

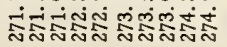

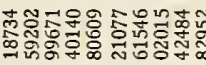

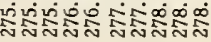

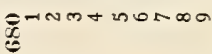

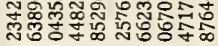

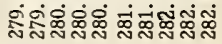

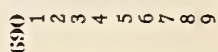

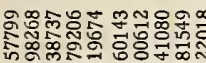

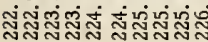
量

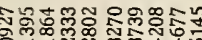

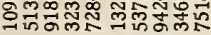

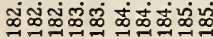
E

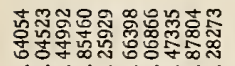

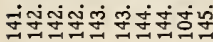

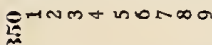

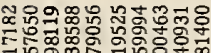

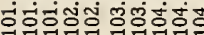

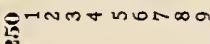
ai

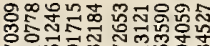
우을

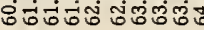
을

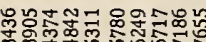

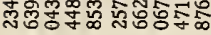

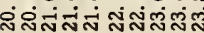

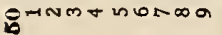

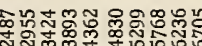
엉구에

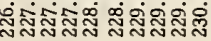

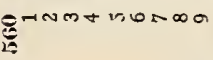

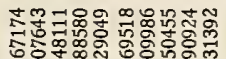

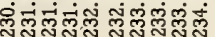
空

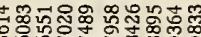

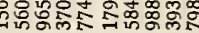

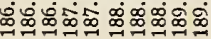

Ornmanona

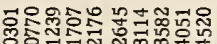
(1)

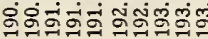

Enmanosia

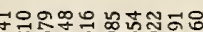

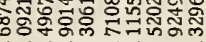

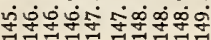

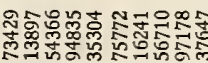

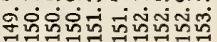

sinmanona

突

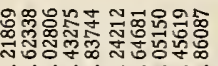

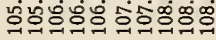

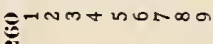

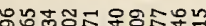

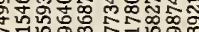

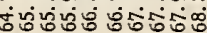

s

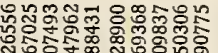

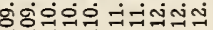

ENnmanono

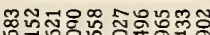

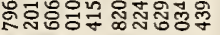

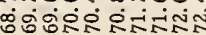
E-Nm+noma

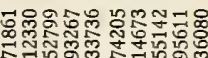

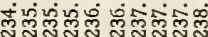
Errnotinona

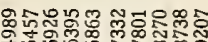

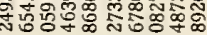

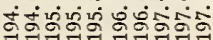
$\bar{E}^{-1 n m+n 日 N \infty a}$

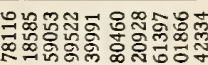

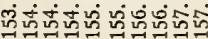
क्रnm+nunda

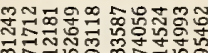

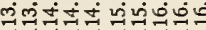

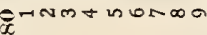

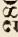

중ㅇำ

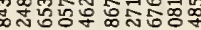

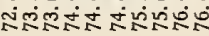

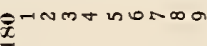

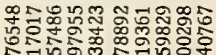

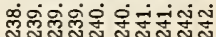

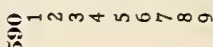

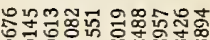

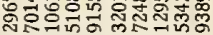

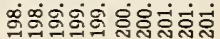

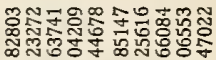

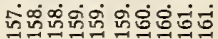
Erratnona

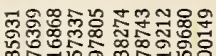
OrNmt nONDa 突

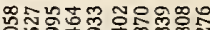

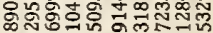

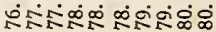

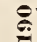

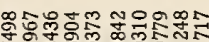

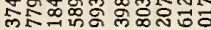

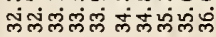
D

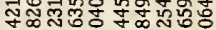

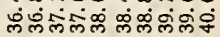

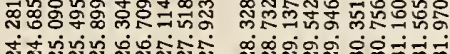

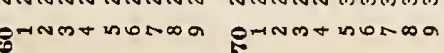

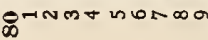

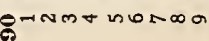




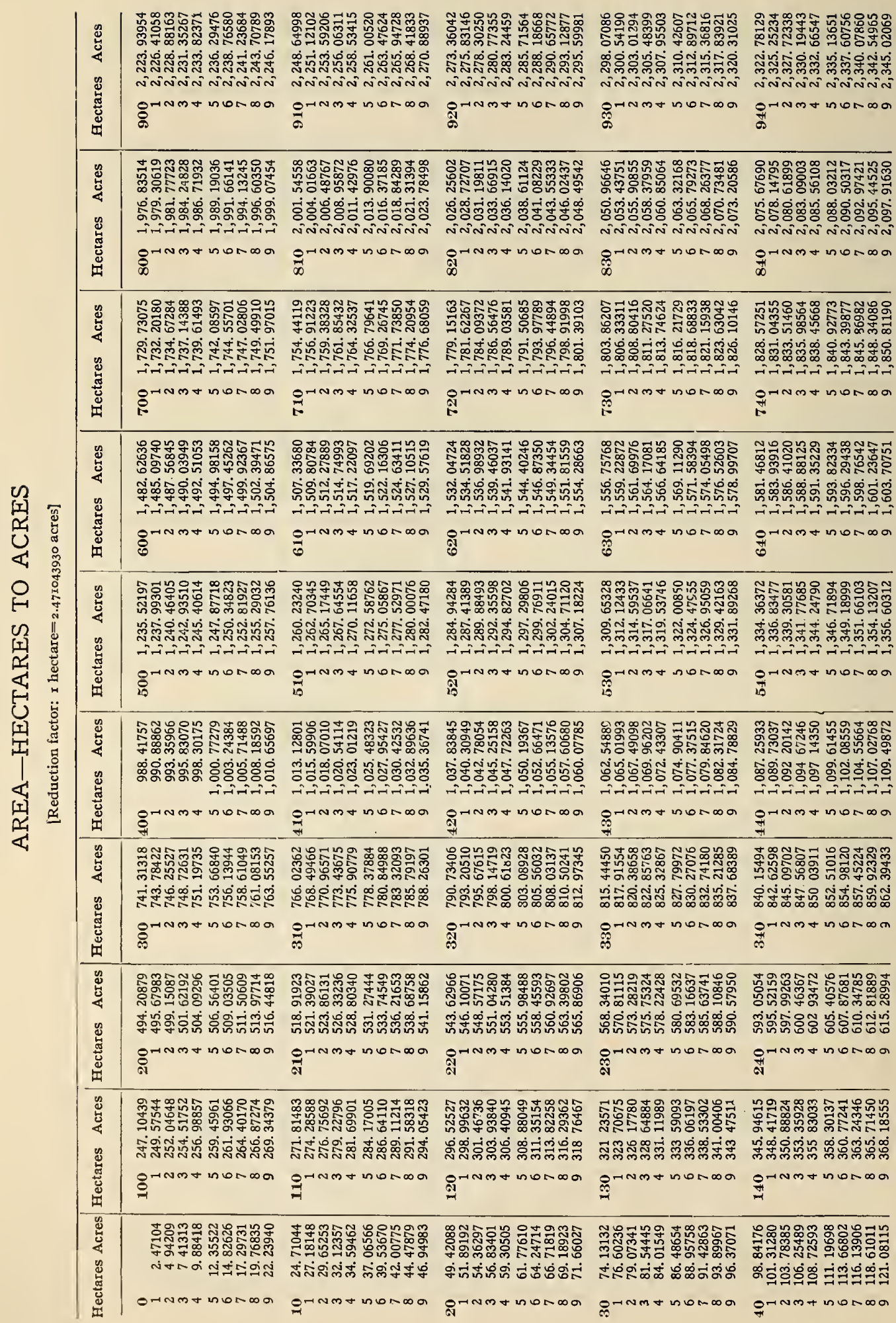


מ⿰纟及卩

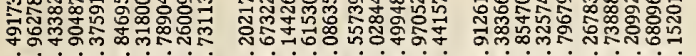

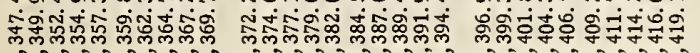

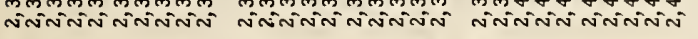

Brnmañona

8

है.

(

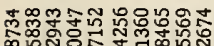

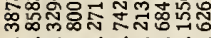

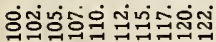

viñviñninivin

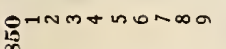

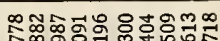

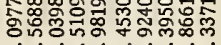

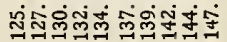

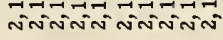

8

Jumanona

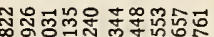

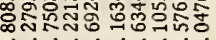

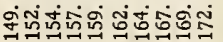

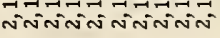

Bnmanona

PrNm+nona

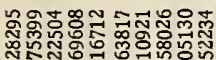

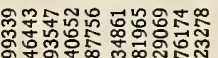

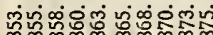

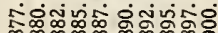

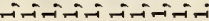

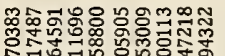

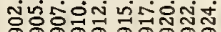

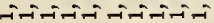

Brnmonono

点

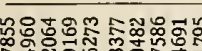

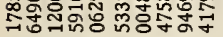

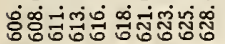

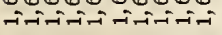

हुतNmonoma

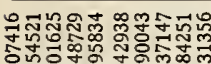

ज्ञ

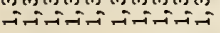

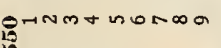

QNNm+LONDO

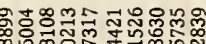

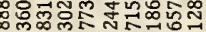

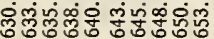

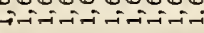

Bringanona

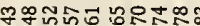

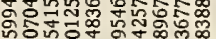
ที่0ல்

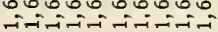

Eramanona

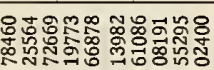

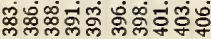

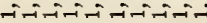

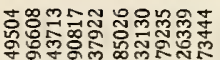

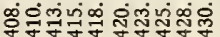

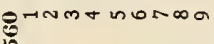

BNm+nona

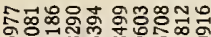

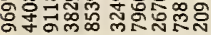

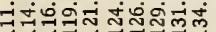

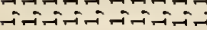

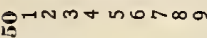

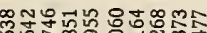

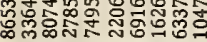

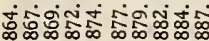

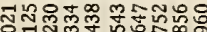

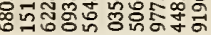

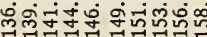

옹요요

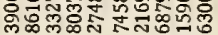

5ं

inifintinit

E-Nmanonom

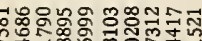

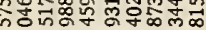

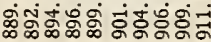

:

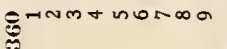

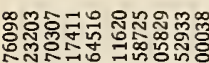

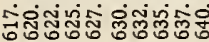

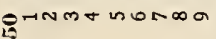

高

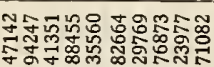

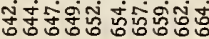

今-nistunana

ifizi-ifizi-

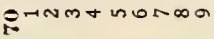

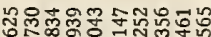

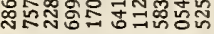

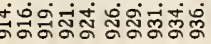

grnmontona

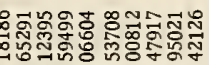

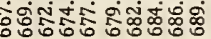

PrNmonuma

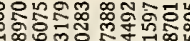

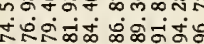

NiniñNininín

$\ddot{x}$

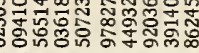

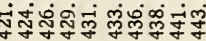

ininininininina

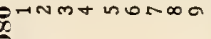

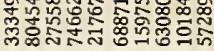

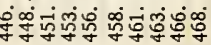

nininininininina

E.

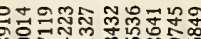

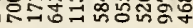

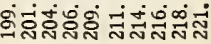

inininininininin

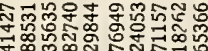

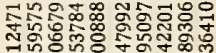

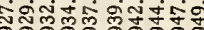

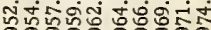

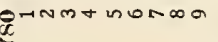

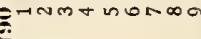

ำㅇํำำำก

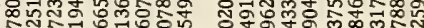

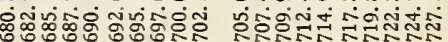

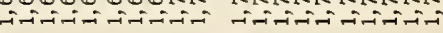

O्रतNm+nONO

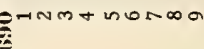

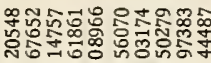

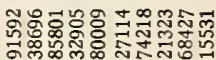

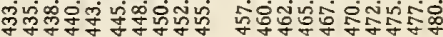

$\rightarrow=-7-1-7 \rightarrow-i=$

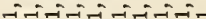

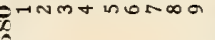

ENm

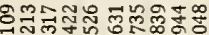

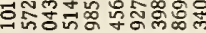

ฒฒ

=-1-i-i-i-i

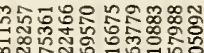

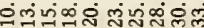

产

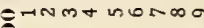

ระ.

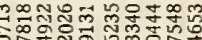

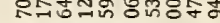

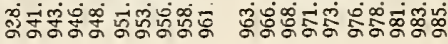

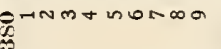

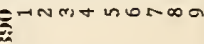

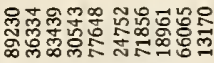

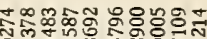

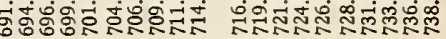

OีNMT RUNDO

ONM+UONDa

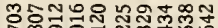

ำํํำ

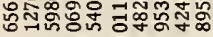

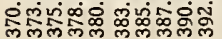

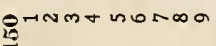

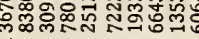

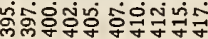

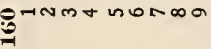

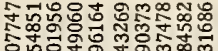

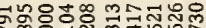

non

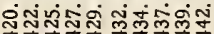

PrNm+nona

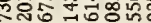

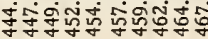

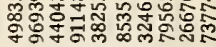

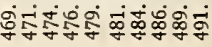

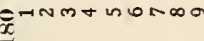

GrNm+nono

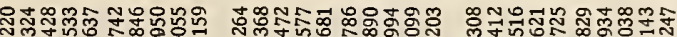

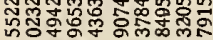

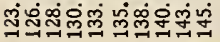

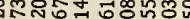

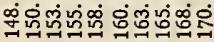

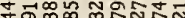

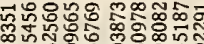

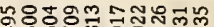

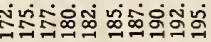

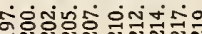

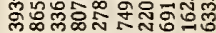

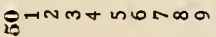

8HNm+nuRna

우요

๑̈rnmanono

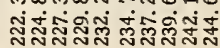

-TNM+ UONDO 


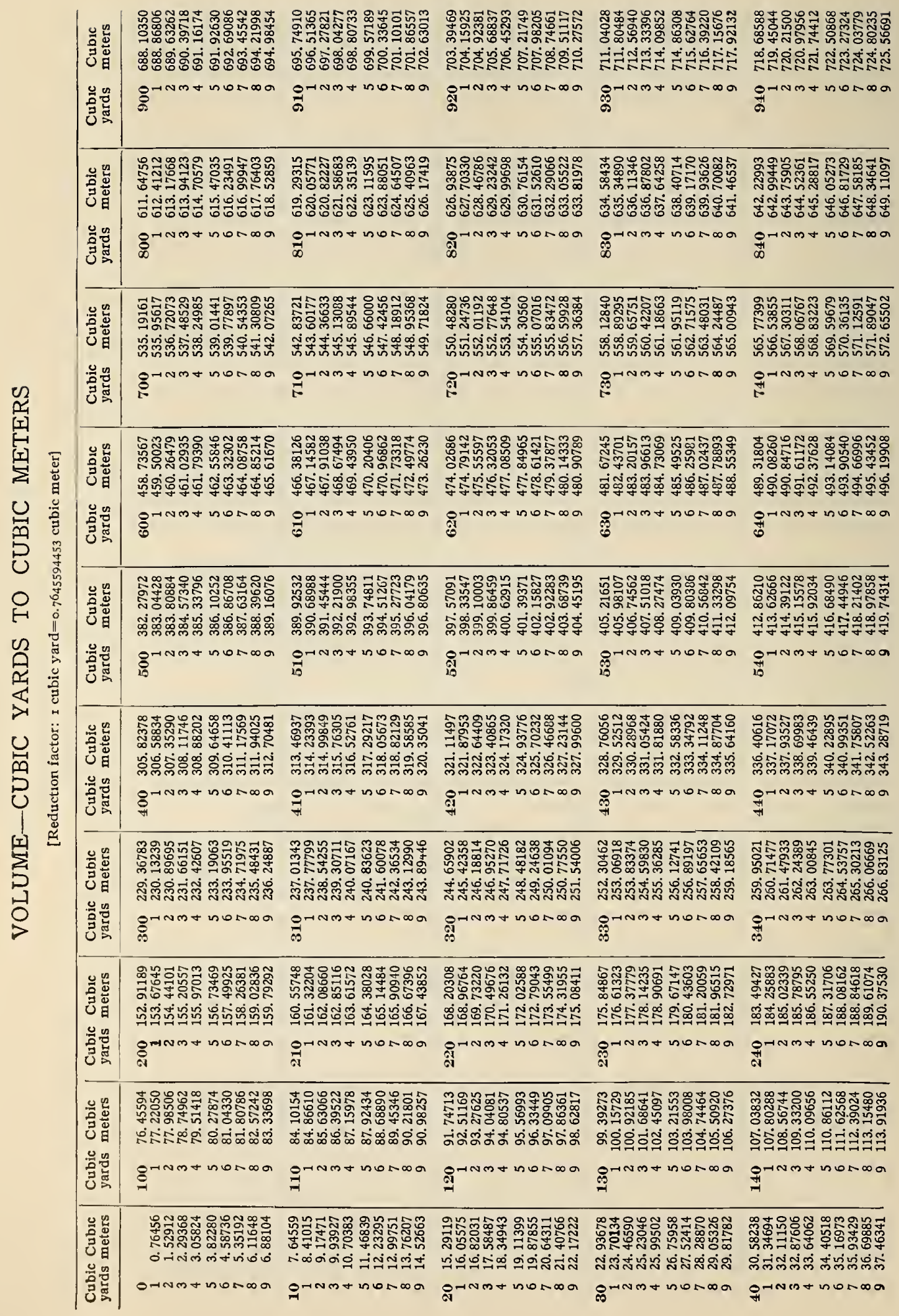




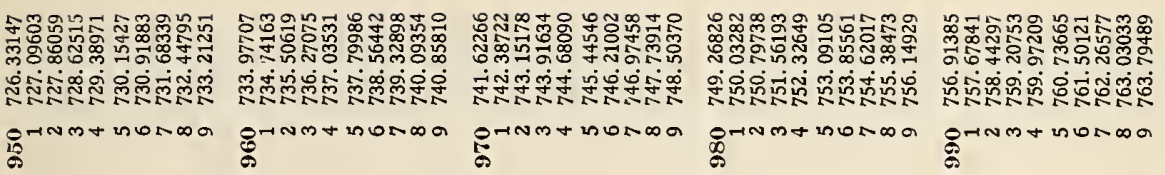

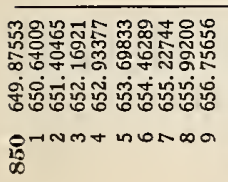

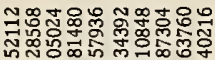

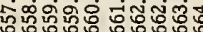

8̈nm+nona

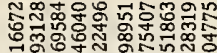

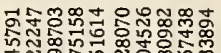

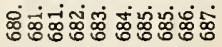

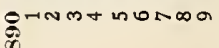

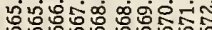

趈

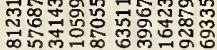

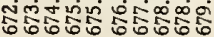

PrNm-nuna

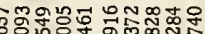

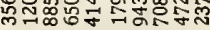

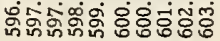
OHNm+nONDa 竞

을

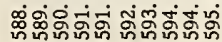

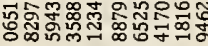

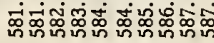

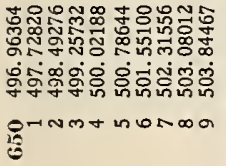

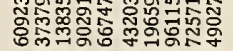

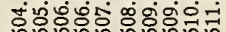

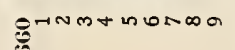

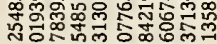
نं लंखं

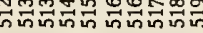
8

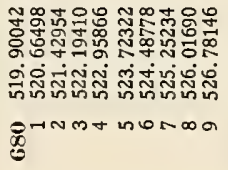

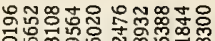

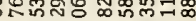

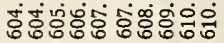

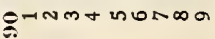

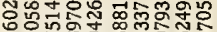

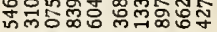

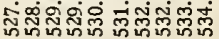
onnestinanosa

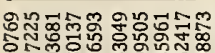
ชुำ

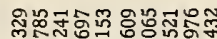

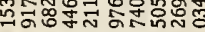

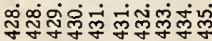

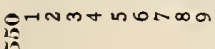

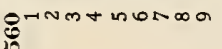

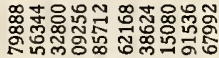

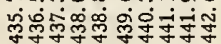
is

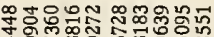

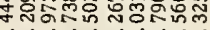

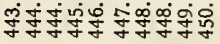

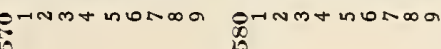

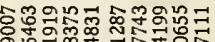
sob

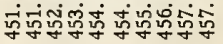

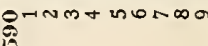

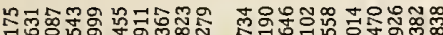
చై

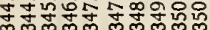

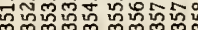

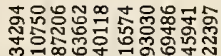

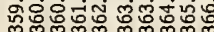

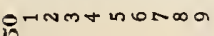

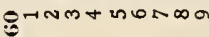
今

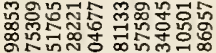

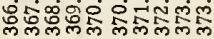

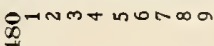
$e^{-1 N \infty+\infty n \text { ono }}$

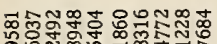

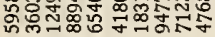

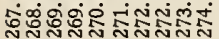

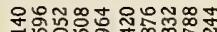

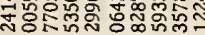

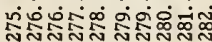

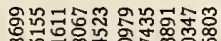

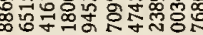

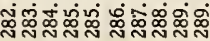

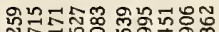

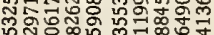
放

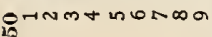
8

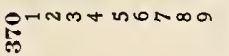

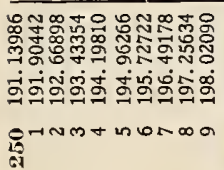

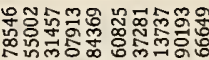

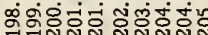

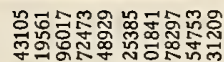

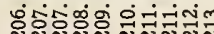

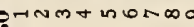
ลิ 옥 要

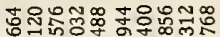

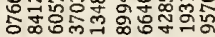

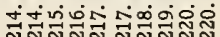

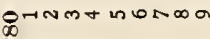
क्ष

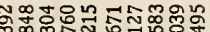

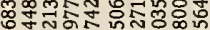

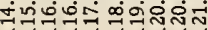

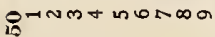

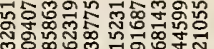

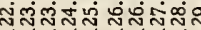

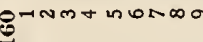

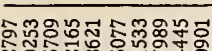

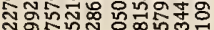

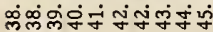
OHNM+ nUNDa

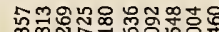

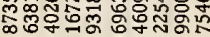

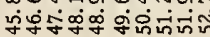

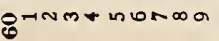

(रrNe+

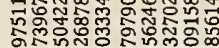

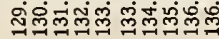
ㅂNm+nona

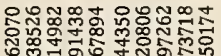

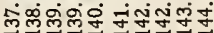

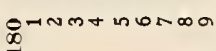

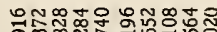

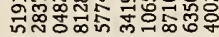

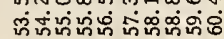
grnosumena

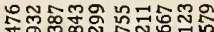

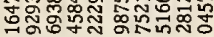

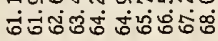

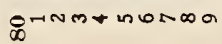

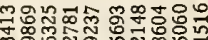
可

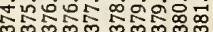
8

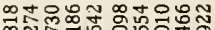

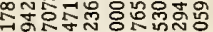
:$$
\text { ent }
$$

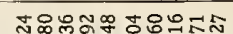
춫:

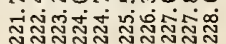

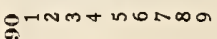
突

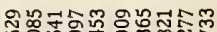

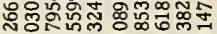

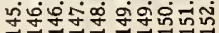

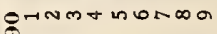
ह

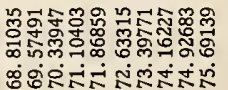

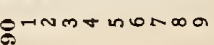




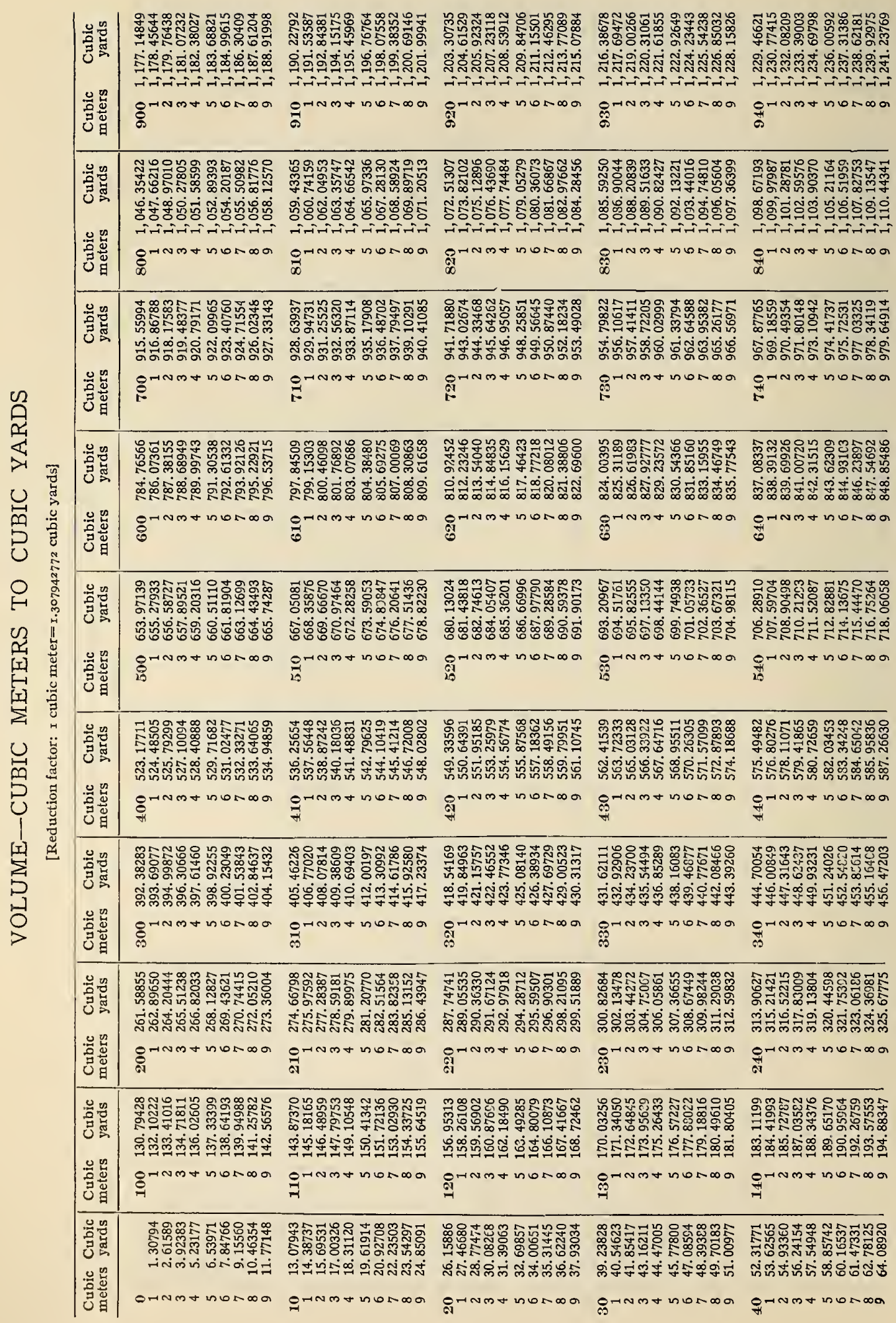




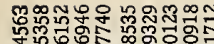

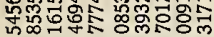

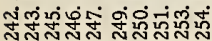

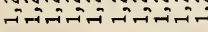
10

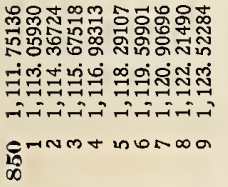

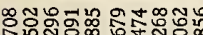

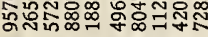

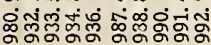

Ponerononom

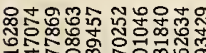

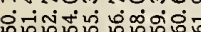

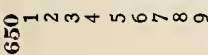

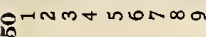

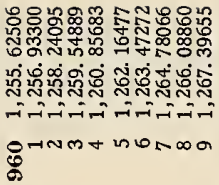

ङ

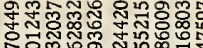

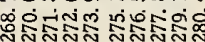

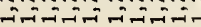

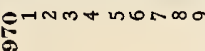

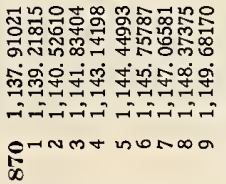

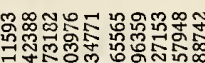

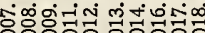

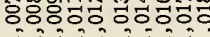
ornmonuma :

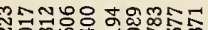

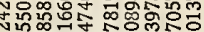

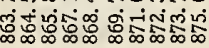
OrNm+nUno

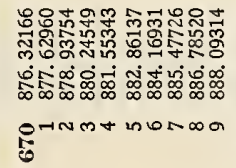

\section{.}

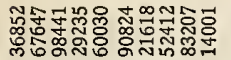

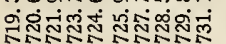
if HNm+ nONDa

\section{(1)}

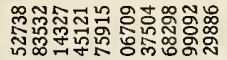

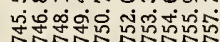
8 O

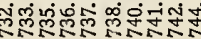
OHNm+nuna

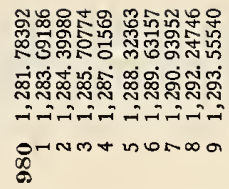

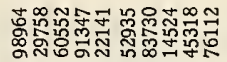
서선

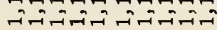
OrNm+nonda

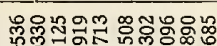

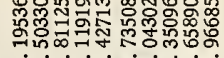

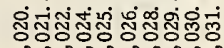

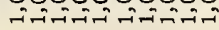
OnNmanoma

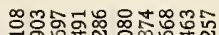

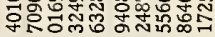

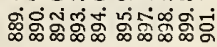
$\mathbb{R}^{-N N m+n U n \infty a ~}$

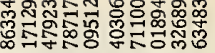

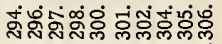
ifiनت है

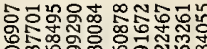

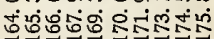

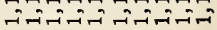
今్

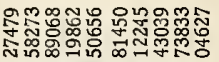

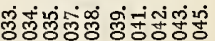
$\because ت ニ=-1=-7=$ ornmantana

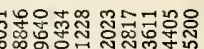

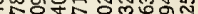

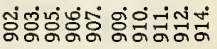

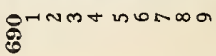

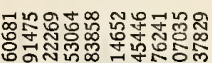

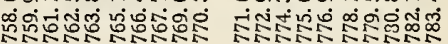
$\infty$ 突

(⿻)

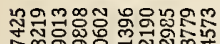

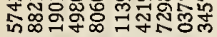

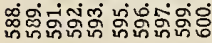

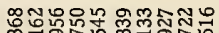

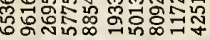

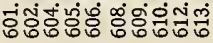

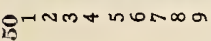

हैं

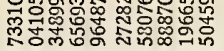

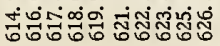

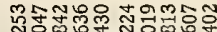

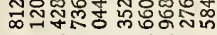

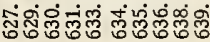

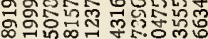

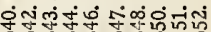

AnMt nUNDa Ornmanuma E-Nmtinuma 赵 牙

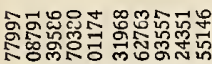

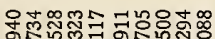

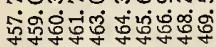

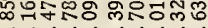

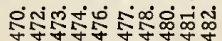

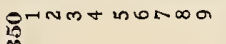

8№m+nuna

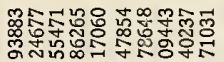

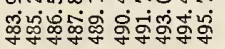

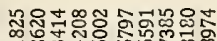

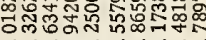

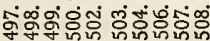
量 (1)

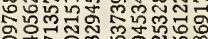

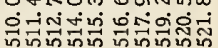

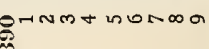

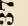

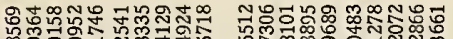

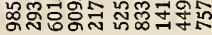

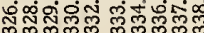

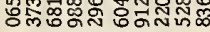

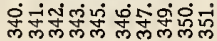

ही Gั

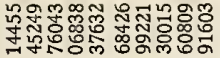

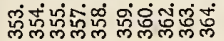

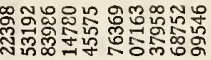
ดீंீ口:

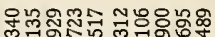

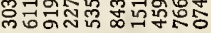

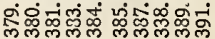

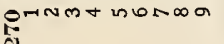

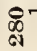

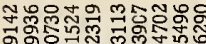

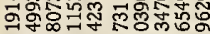

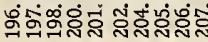

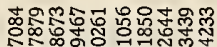

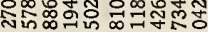

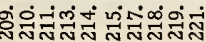

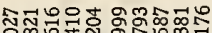

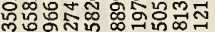

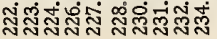

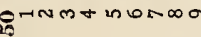
8

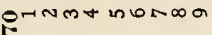

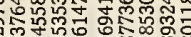

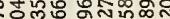
ம்ळ்

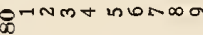

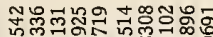

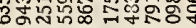

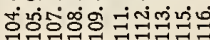
किष

8ृतm 尺 बิ

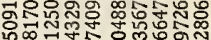

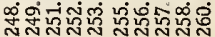
8

OrNmantana

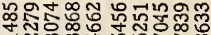

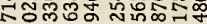

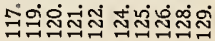
8 


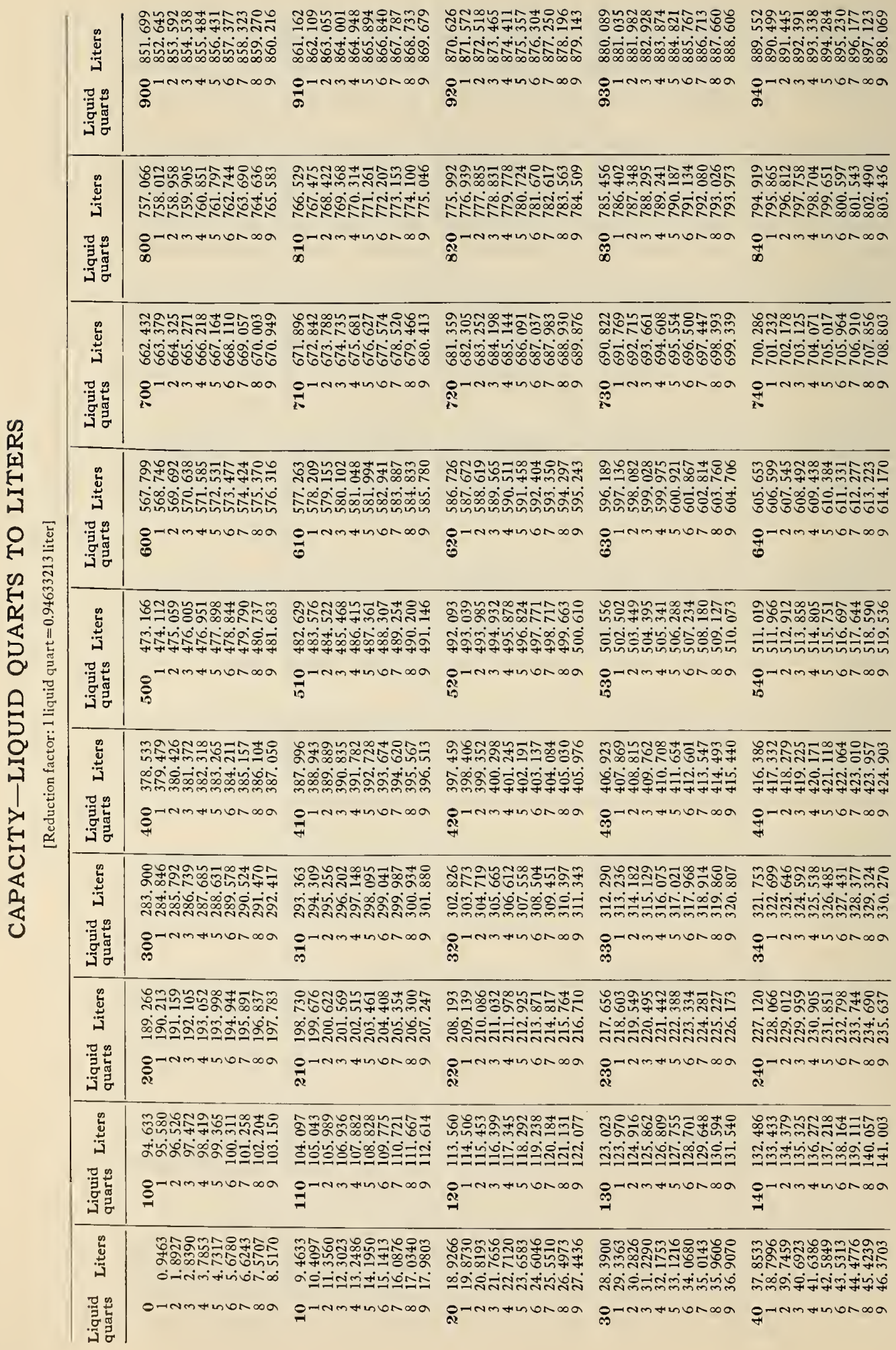




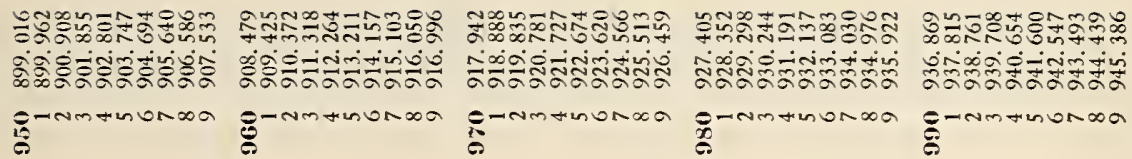

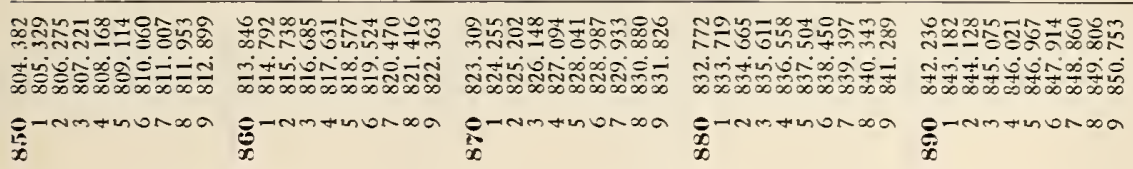

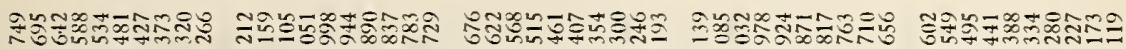

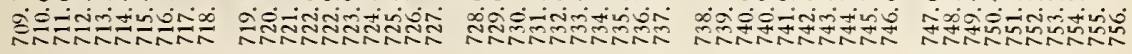

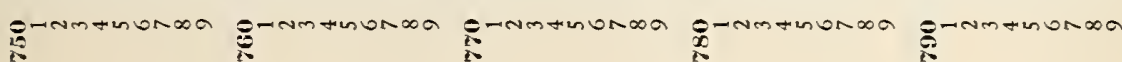

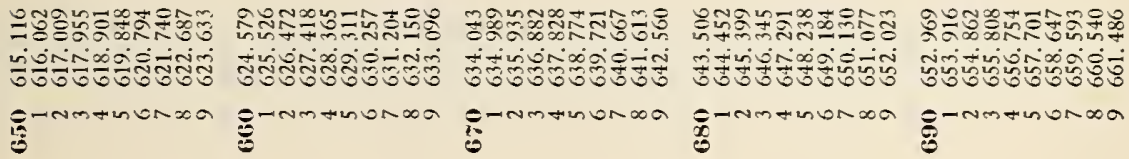

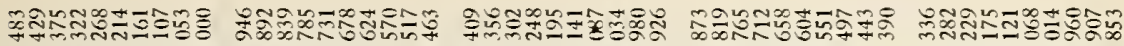

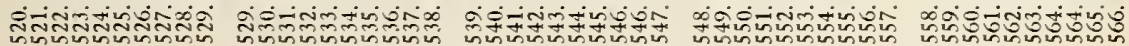

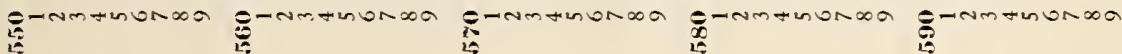

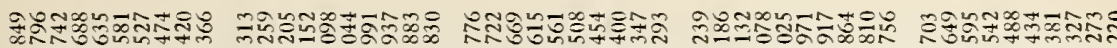

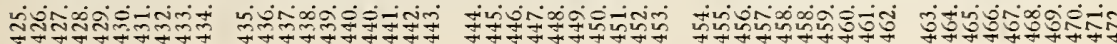

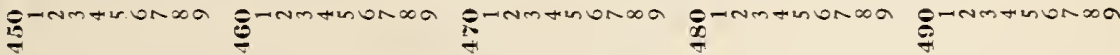

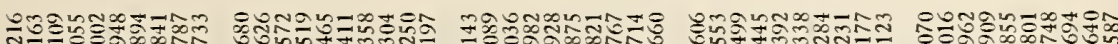

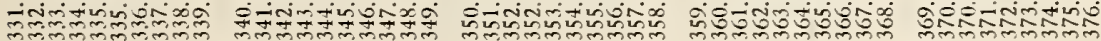

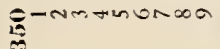

产-nmanonos

हैंतmanonod

indinona

e-notinonos

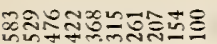
Sin $x$ a

(1)

Oi.

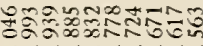

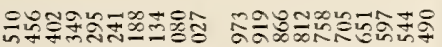

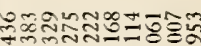

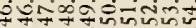
พñ

o-nmanonom

- - nmtinunomo

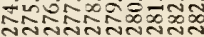

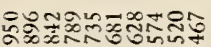

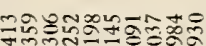

orraminonion

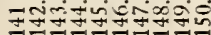

inis

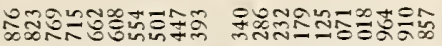

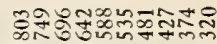

E-Nm+LnONon

E-Nmtinonoa

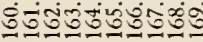

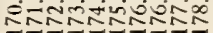

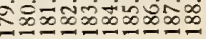

E-rmtinondon

O-nmtinunoma

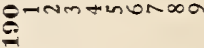

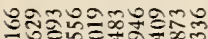
Ni

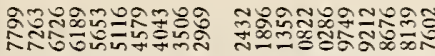

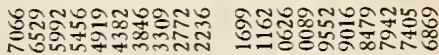

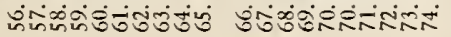

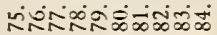

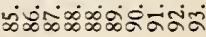

जิ

e-nmanumsa

O-rmanunda

ennminonda 


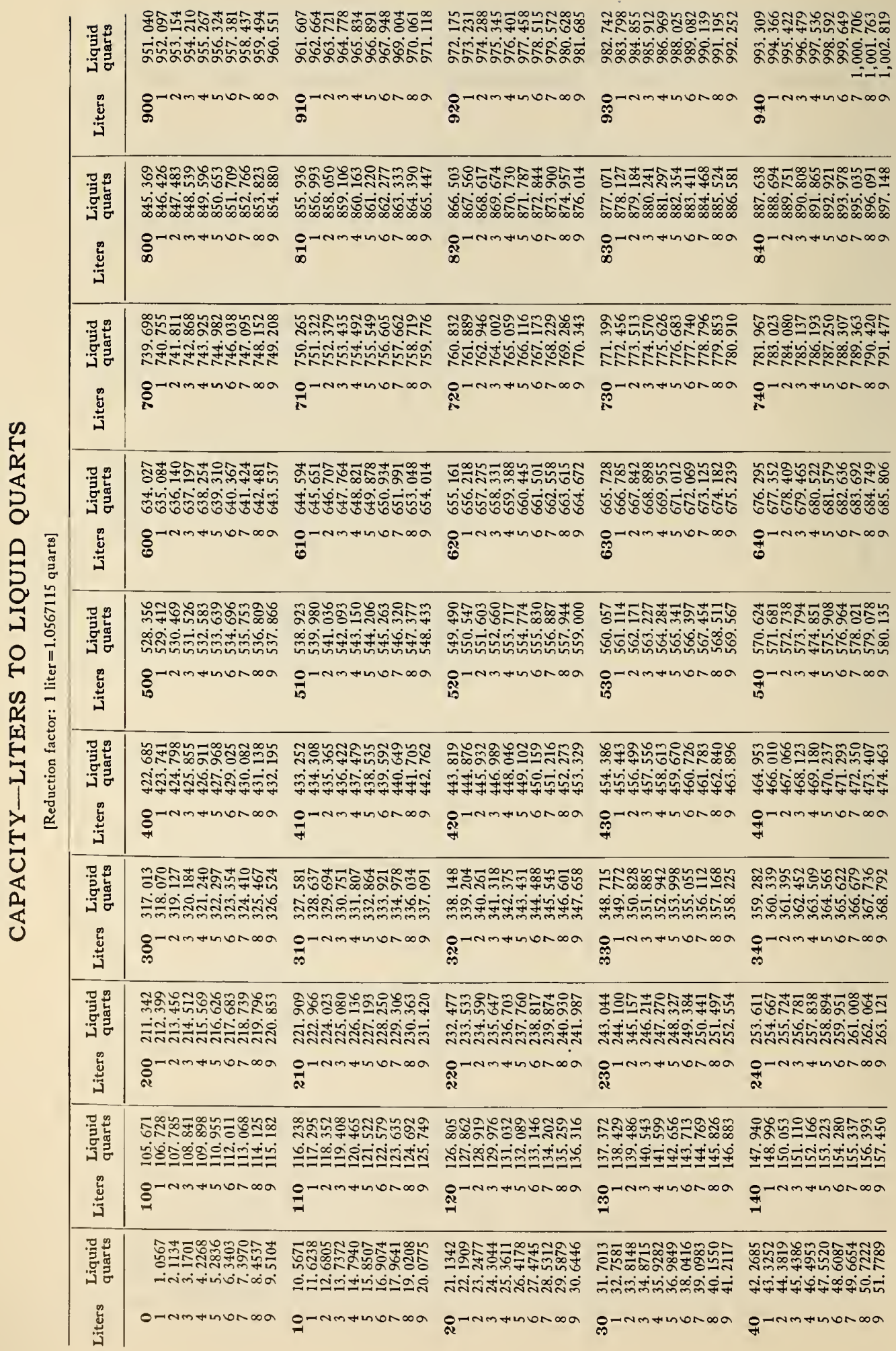




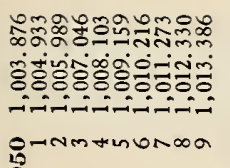

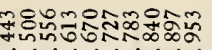

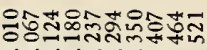

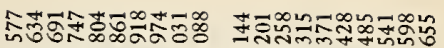

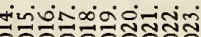

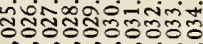

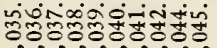

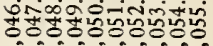

Ërnanonosa

8̈rntinonoma

D-Nmanonosa $^{\infty}$

8-nmtinonosa

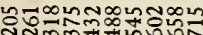

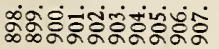

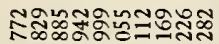

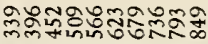

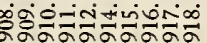

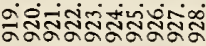

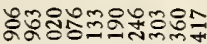

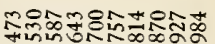

Oि-Nm+inunon

ङ

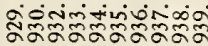

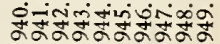

हnisionosa

Drrmanundo

甯

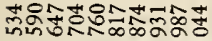

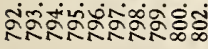

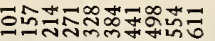

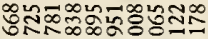

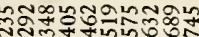

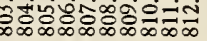

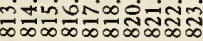

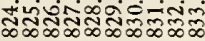

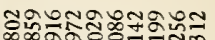

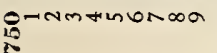

:-Nm+inunaa

运

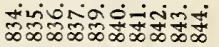

g-nmanunoa

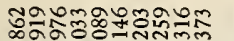

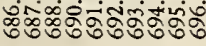

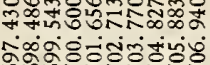

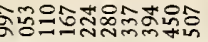

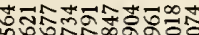

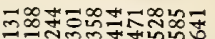

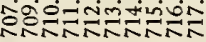

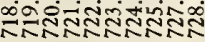

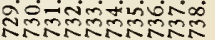

8

8

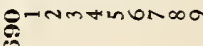

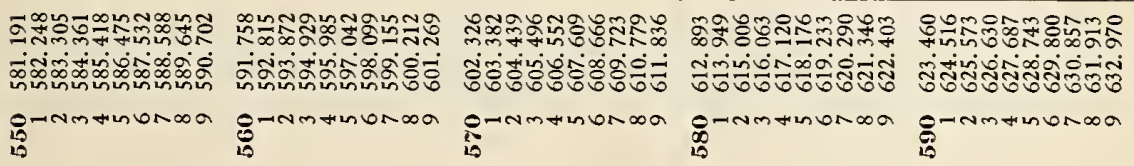

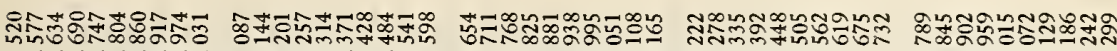

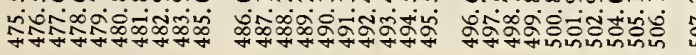

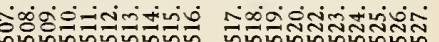

Ërnmanonon

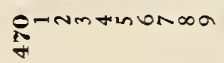

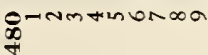

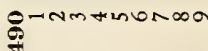

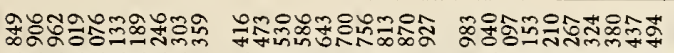

(2)

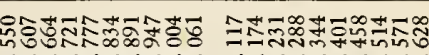

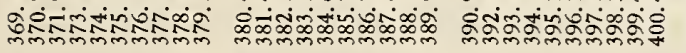

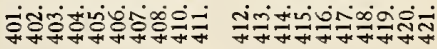

is

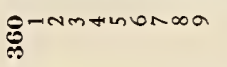

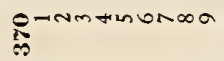

OD-nmtinona

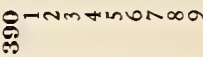

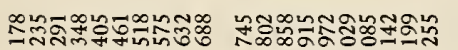

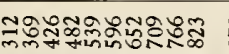

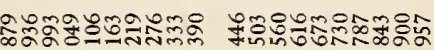

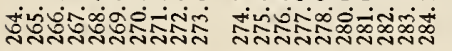

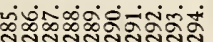

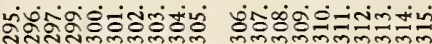

这

8.

$\underset{R}{R}$

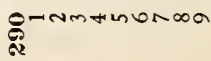

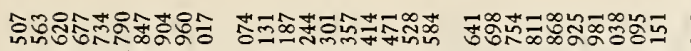

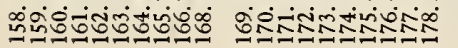

iिं

送

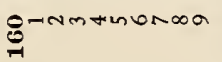

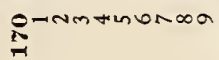

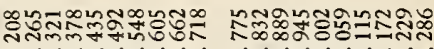

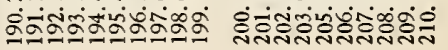

o-nmanumasa

-NMtin un

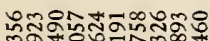

कom

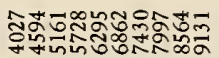

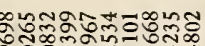

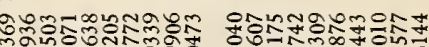

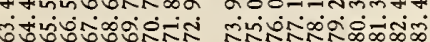
भin

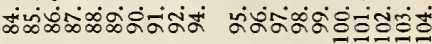
:

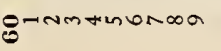

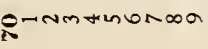

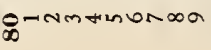

g-nmtinuma 


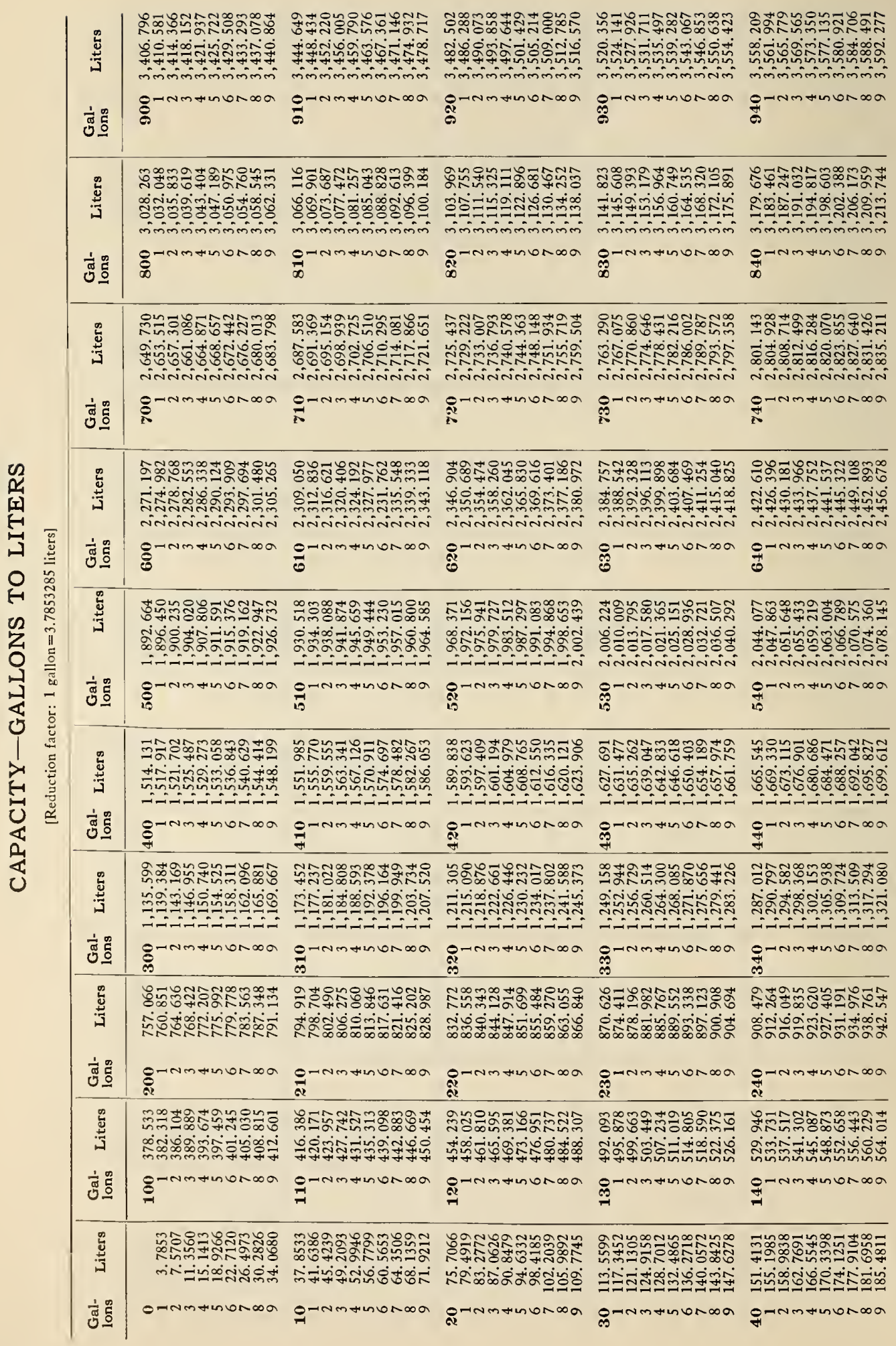




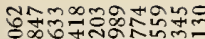

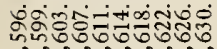
miniminiminiminom

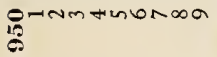

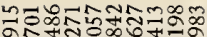

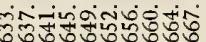
O3. \&̈rnminonasa

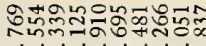

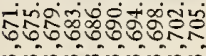
是

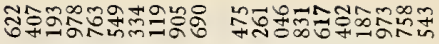

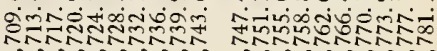

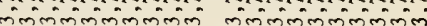
Orraminonosa

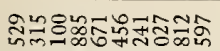

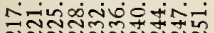
minminiminimino

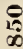

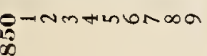

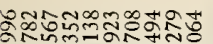

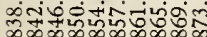
inininininiñ

:

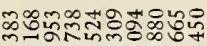

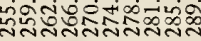

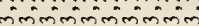
ఠ్

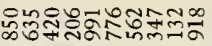

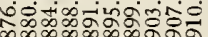
ininininininin

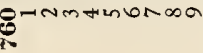

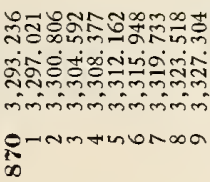

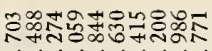

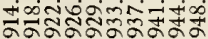
inininininisin 용

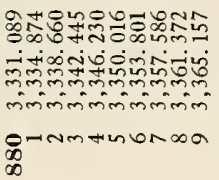

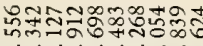
นं宀 ininininininín

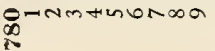

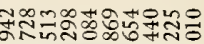

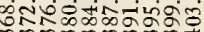
niminiminimiñ

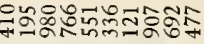
ธํํㅎำ iniviminimin-

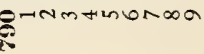

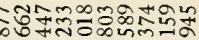
تnंgiñ

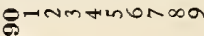
ळ

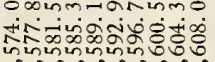
ninininininina ह

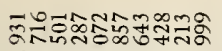

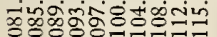
niniñniññNín E्रmantina

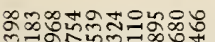

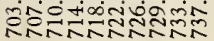
RNA

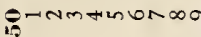

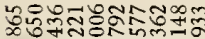
संक्रिण

=

O-तmanur

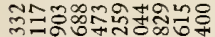

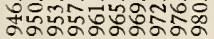

G-Nmtintoroa 10

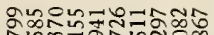

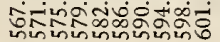

Prinmtintona

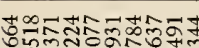
Nonuf

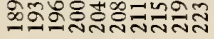

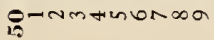

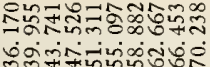
in 尽-Nm+non

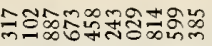

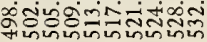
rinininininina हैतmantina

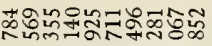

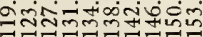
iñNininininín êtrmtinbina

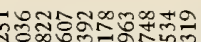

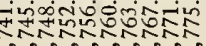
8 8

\section{कtran}

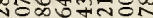

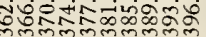

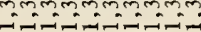
$\infty$

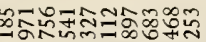

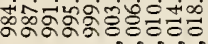
ベーニー-

E-Nmtingnoa

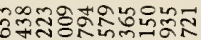

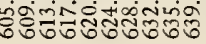

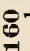

O-Nm+1nण $\infty 0$

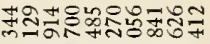

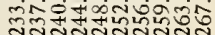
ininaninarina 8.r.

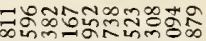

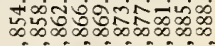

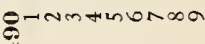

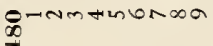

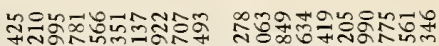

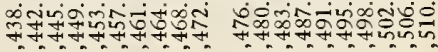
O)-Nmtinon $\infty$ O-NMtinono

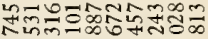

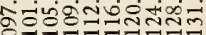
는?

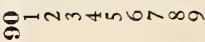

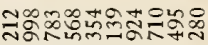

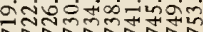

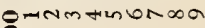
$\infty$

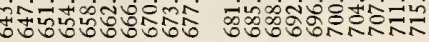
O-Nm+in $-\pi \infty a$

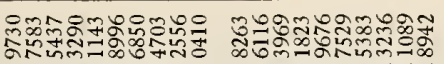

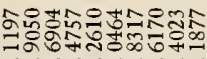

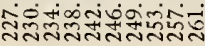

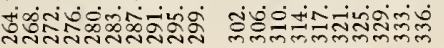

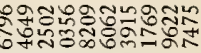

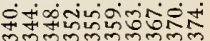
हु-तmtinणn

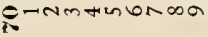

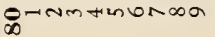




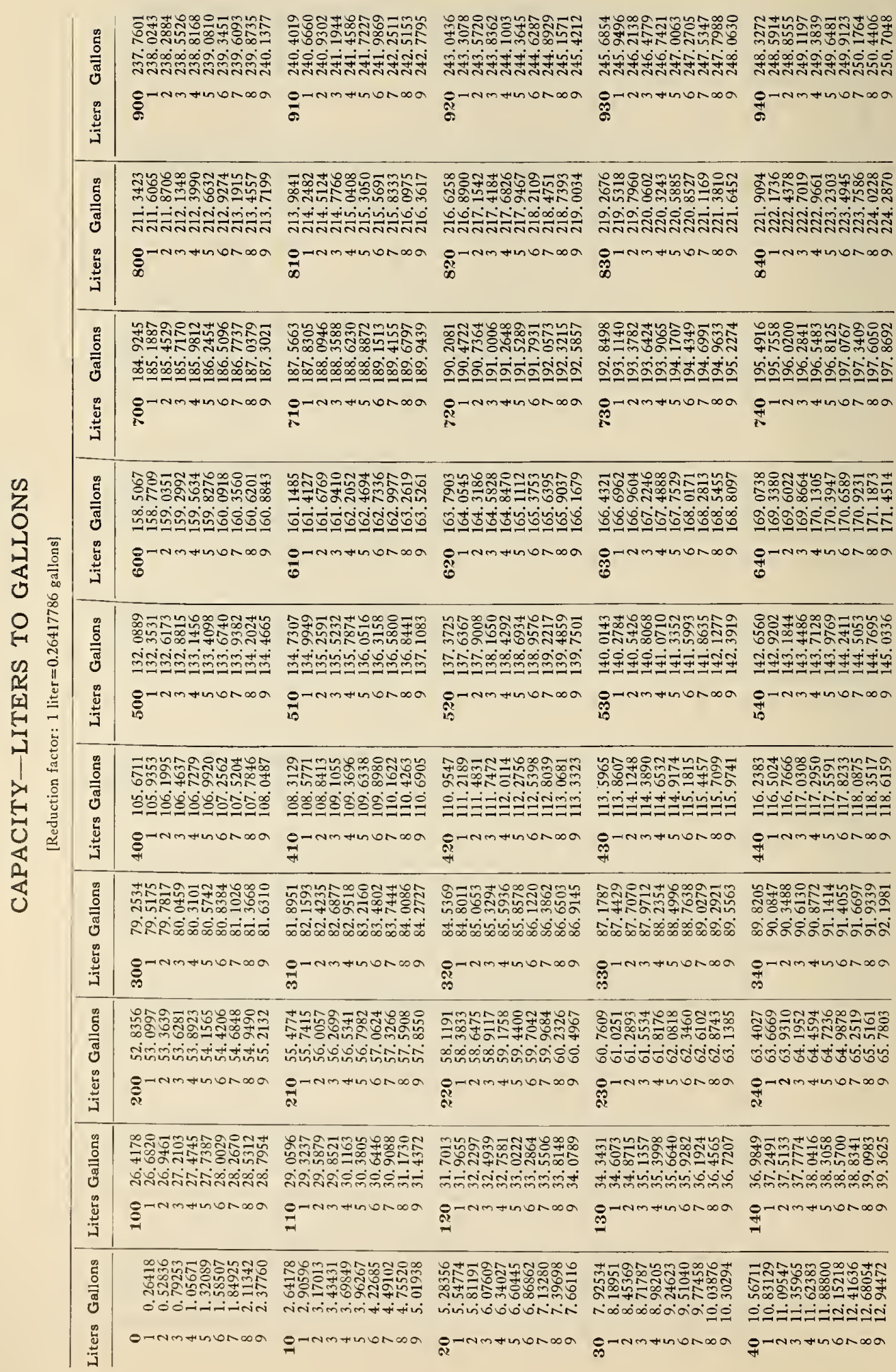




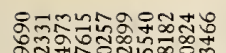

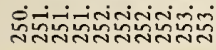
ㅁrvmantora is

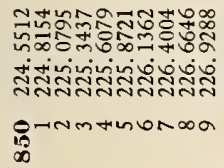

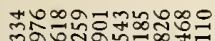

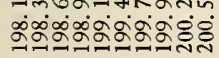
:

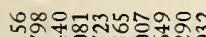
mรำ

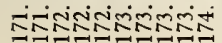
Oด-Nmtinumon 19

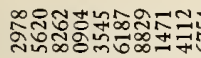

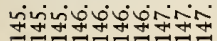

(․․․

-rentuntoroa

(2)

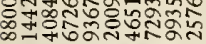

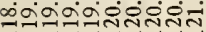

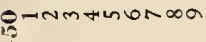

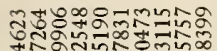

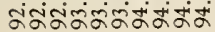

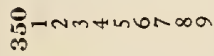

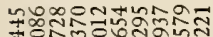

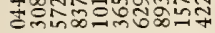

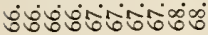

- -Nmtin 0 (2)

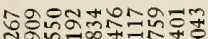

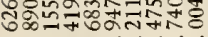

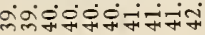

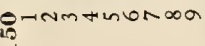

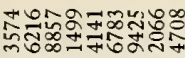

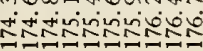

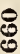

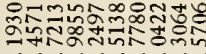
ส สं

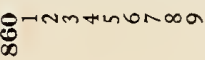

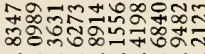

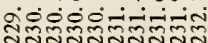

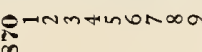

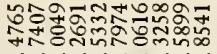

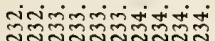
$\infty$

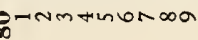

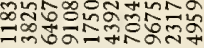

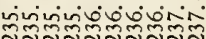
g-Nm+4niono

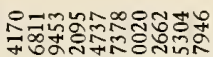

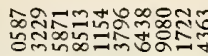
邹

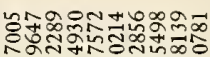

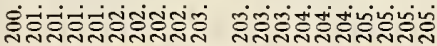
Orramtinunoa

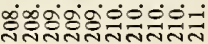

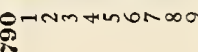

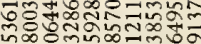

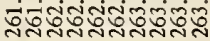

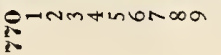

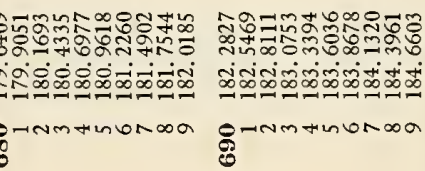

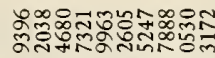

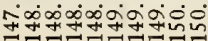

Erraminoma

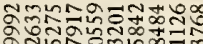

年

R-nmtinuna

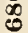

8

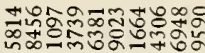

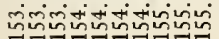

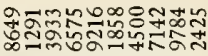
wisinivivivive

g-Nmanonosa

O-Nm+inoroma

8-Nm+ाँणno

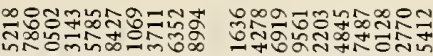

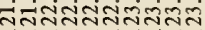

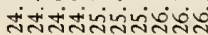

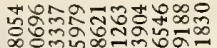

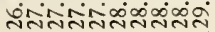

8-rintinuma

욧nmtintoroo

O-Nm+inorioa

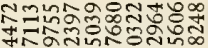

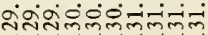

హ.

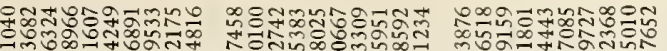

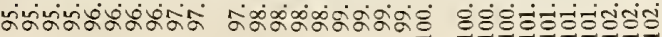

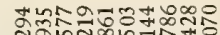

O-Nm+inoria

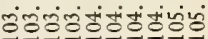

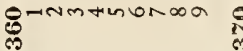

Q-Nmtintoroa

ด्Nm+in

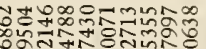

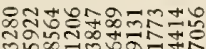

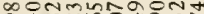

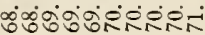

זהזהतהismin

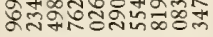

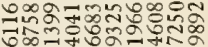

8-Nmtinuma

P-Nmtinbono

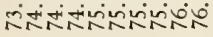

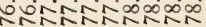

ब

क)

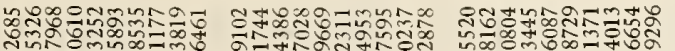

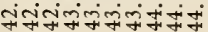

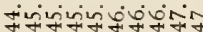

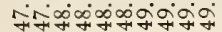

g-ramanorna

은

怘

aำ

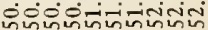

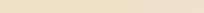

을

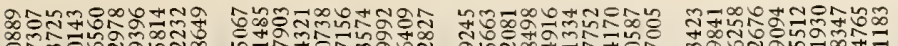

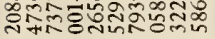

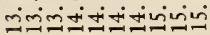

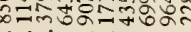

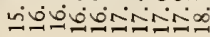

भกำ

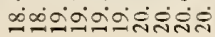

i-

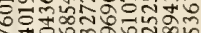

O-Nm+inoroa

8-Nm+inorora

Prrmantono

OD-Nmtintor

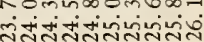

O-NM⿻上丨冂 


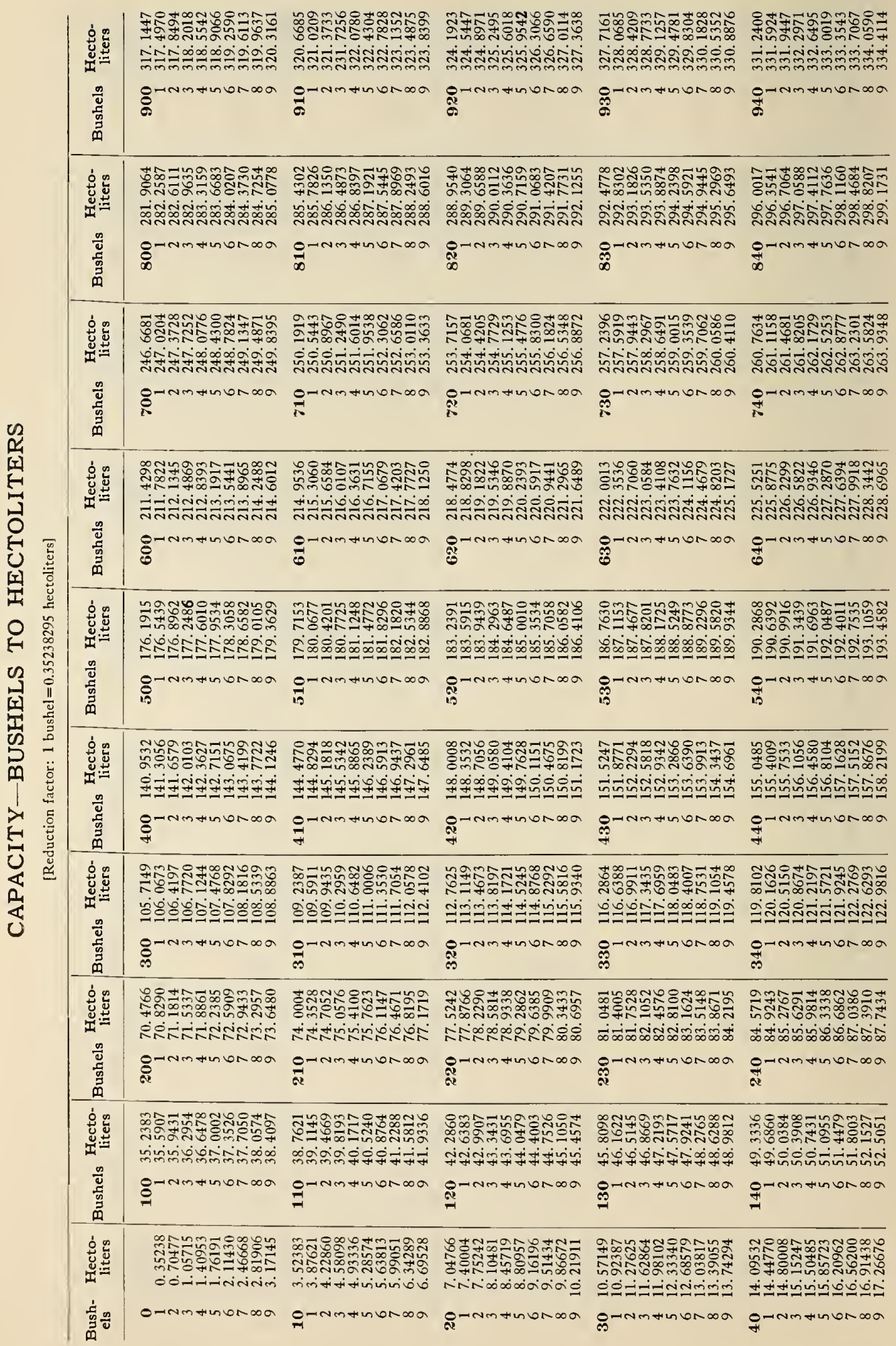




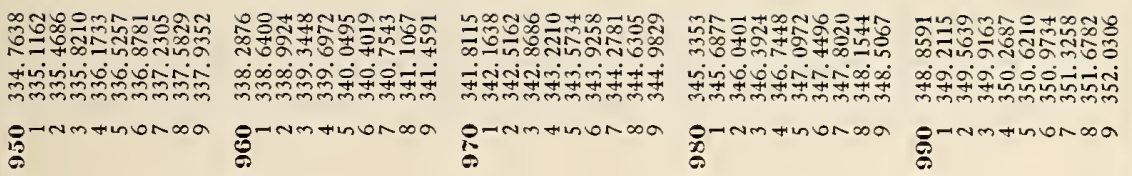

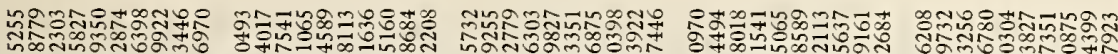

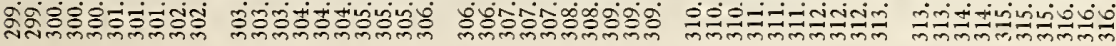

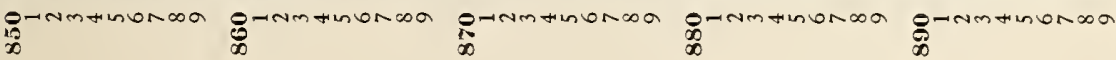

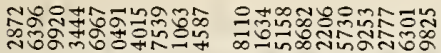

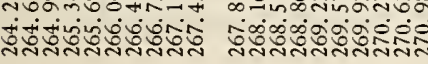
竞8-Nminonom कीm-

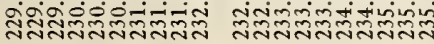
: है

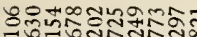

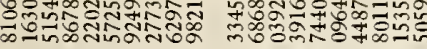

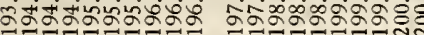
号

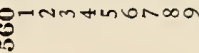

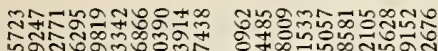

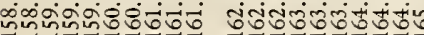

彜

-nm+inton $\infty$

g-nmtinoroo

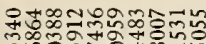

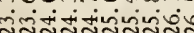

ถัก

(n)

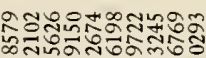

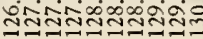

O-Nm+in 0 -

ผื⿻ +क

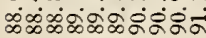

in

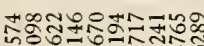

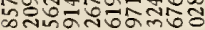
तี

15

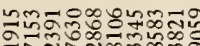

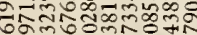

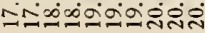

10

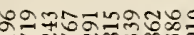

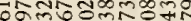

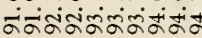

g-nmanono

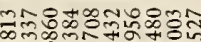

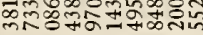
นึinisinisinisio Printinono

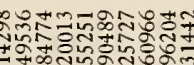
भक्षल

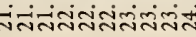
8

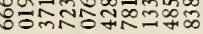
栗

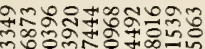

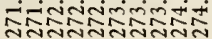
Prrmatinonos

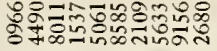

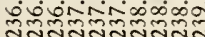
grnmantinaa

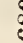

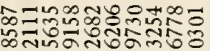
inninisivis OPNm+inonosa

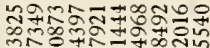

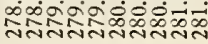
g-Nmtintono

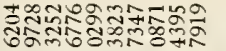

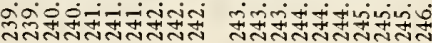
$\mathbb{D}^{- \text {-mmationosa }}$ g-Nm+intono

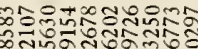

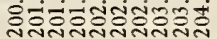
errmatinonoo

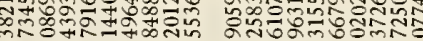

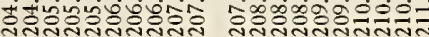
$D^{\text {DrNmtinonoma }}$ 8

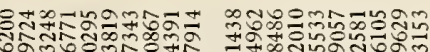
मivo

R-Nmtinona

g-ventintona

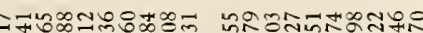

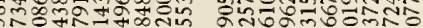

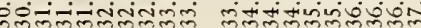

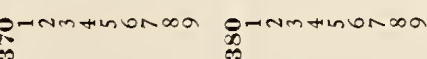

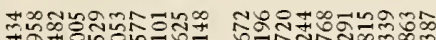
舟

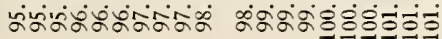

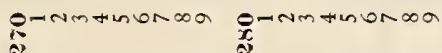

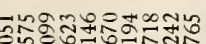

รूली

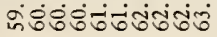

ENAmtiniona

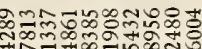

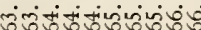
OD-Nmtin

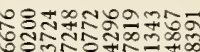

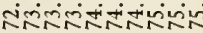

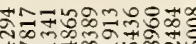

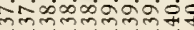

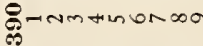

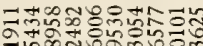

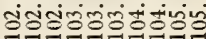
है

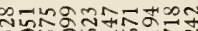
m.

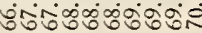
हुmmtintoroa ซำํำกำำำ

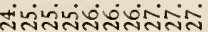

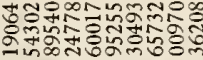

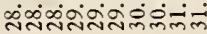

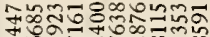

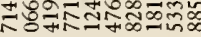

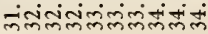

8 


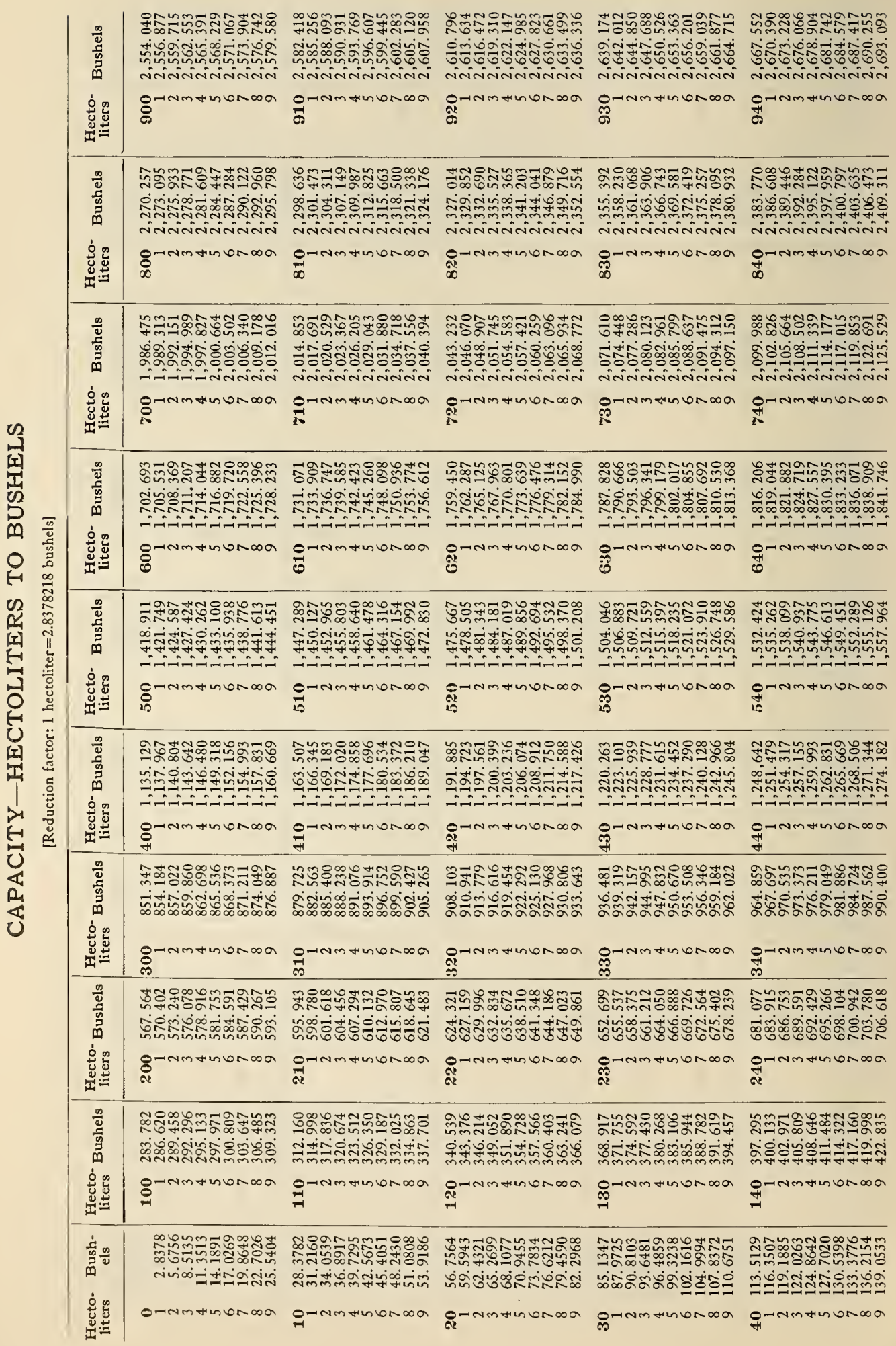




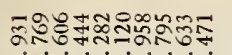
tho तininninininiñ

ำ

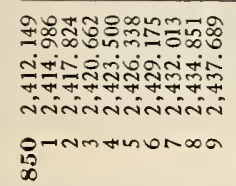

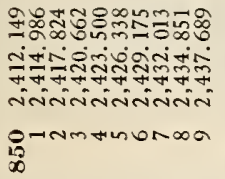

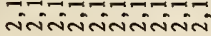

\section{突}

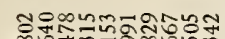

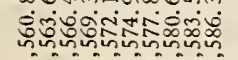

8-Nmonoria

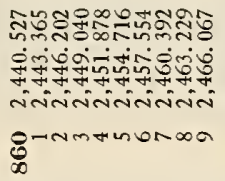

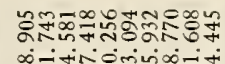

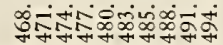
inivinininin $\underset{\infty}{8}$

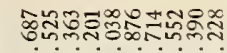
Tiniontgiginin

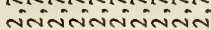

8

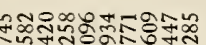

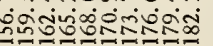

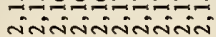
8 visiovinitio

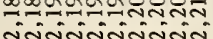
果

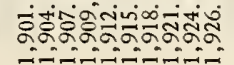

:

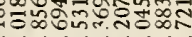

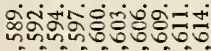

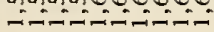
8)-Nmanona

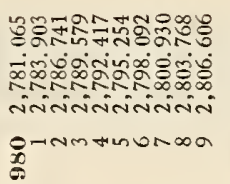

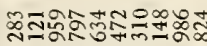

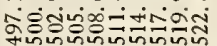
inininiviniti

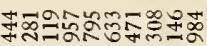

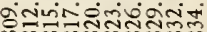
vininininivini g-rimtrunorora бํำผ तुં ninininininis g-nmanuna

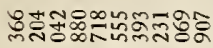

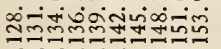

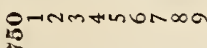

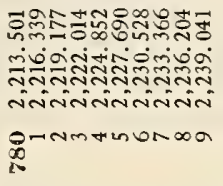

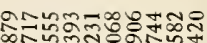

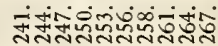
तininininivinit g-Nmmintona

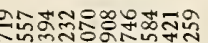

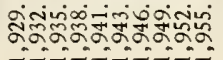

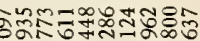

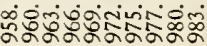

8

8

grnmanonoa

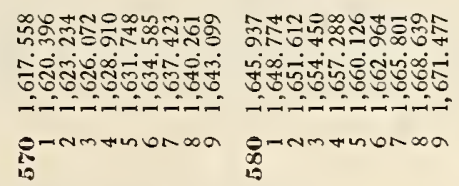

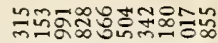

ชิํำ

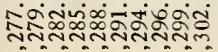

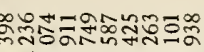

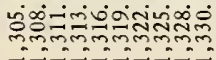

B-Nmtintono 8

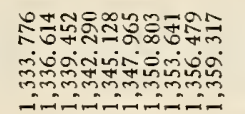

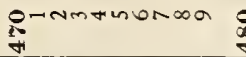

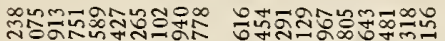

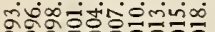

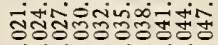

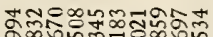

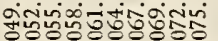
o.

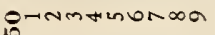

is 8-nmanuma

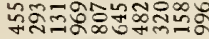

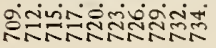

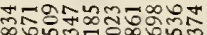
里

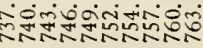

ลิ

है

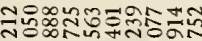

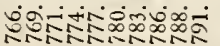

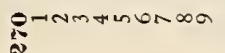

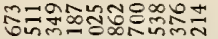

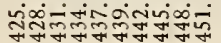

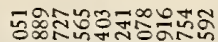

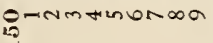

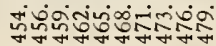

हु-

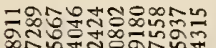

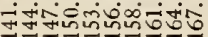

P-Nmantona

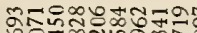

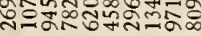

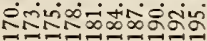

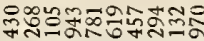

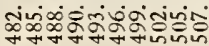

g-Nmanuno

8-rimtinor

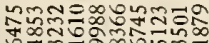
ڤंड் Q-NmWnonor $\infty$

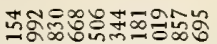

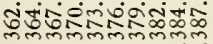
.........

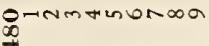

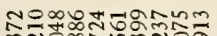
ธิం-

-nin-miñ-

O-rumtinonoa

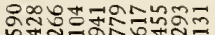

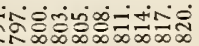
象

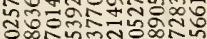

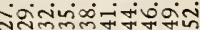

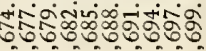
g-nmtinonoma

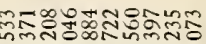

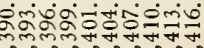

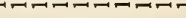

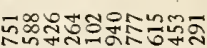

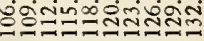
$\because=:=-1$ g-nm+rnonon

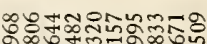
तinioj-iñinino g-nmtinun $\infty$ a

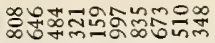
อิO-Nmtinuna क

ฉ

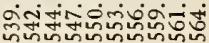
8

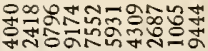
vioजत्रत्तलतNतNล g-nmनाnणna 


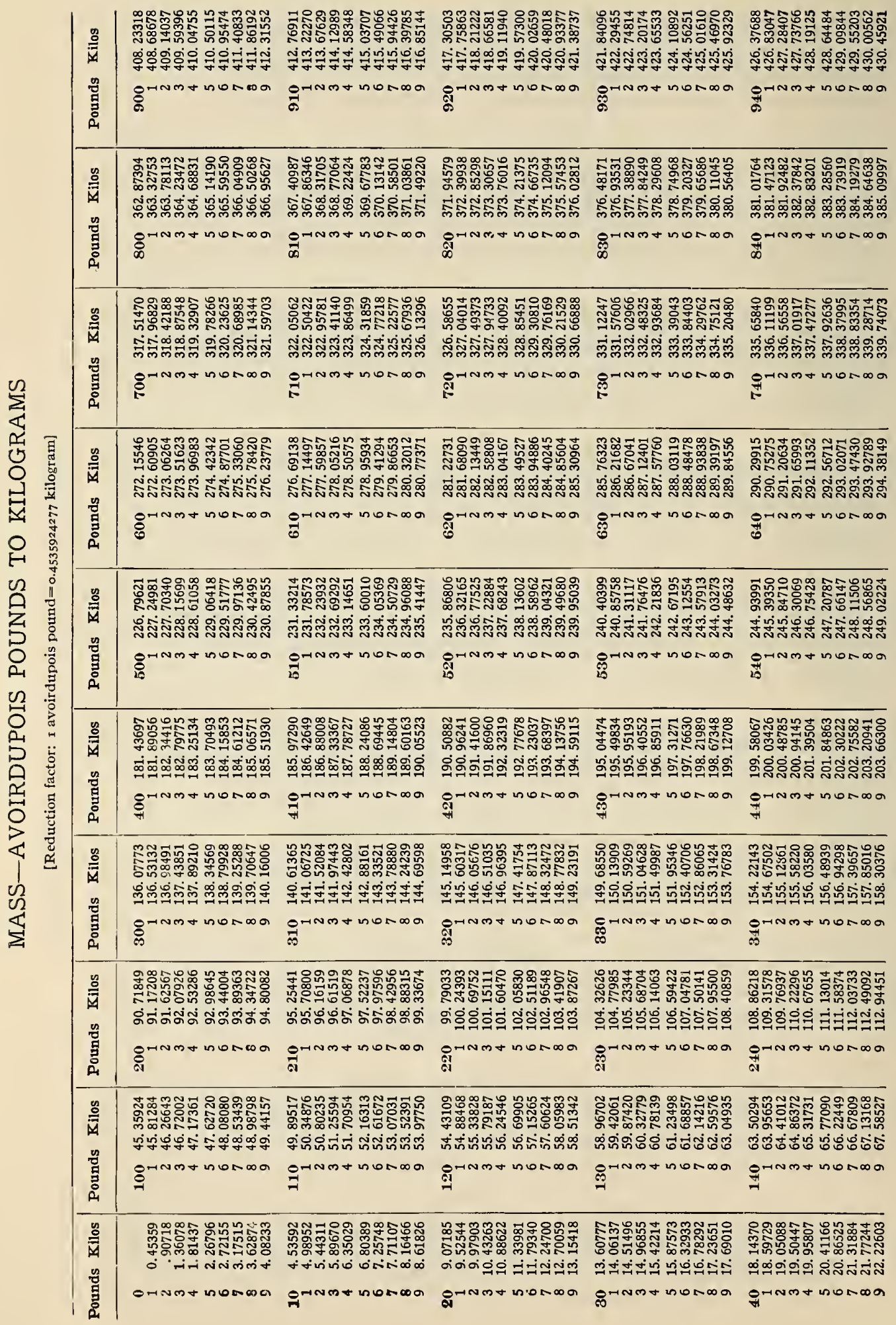




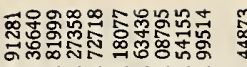

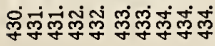

คี

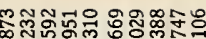

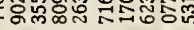

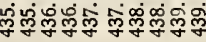
छ

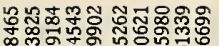

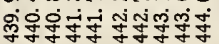

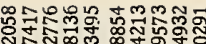
जor

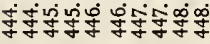

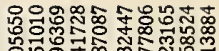

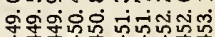

OHNOTHONO

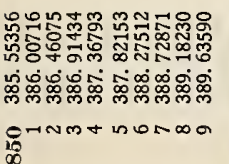

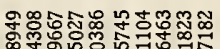

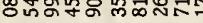

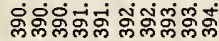
\&్

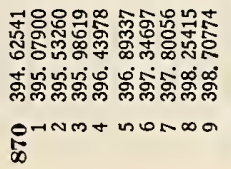

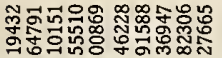

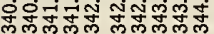
은

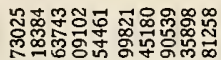

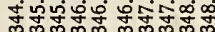

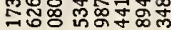

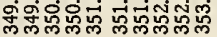
是

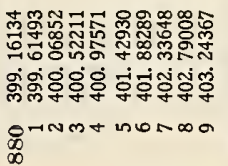

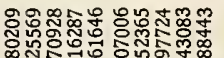

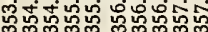

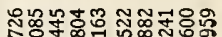

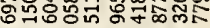

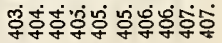
零

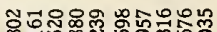

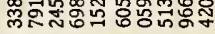

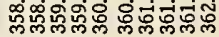
Prnmanoma 央

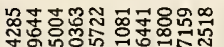
$+\infty \mathrm{m}$

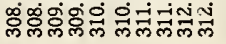

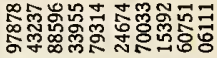

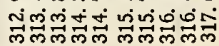

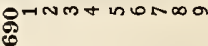
PrNmtunana $\mathscr{D}^{-1 N m+n o n a ~}$

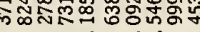

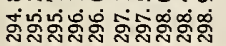
है हैं

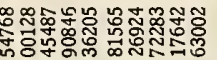

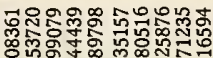

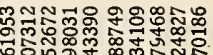

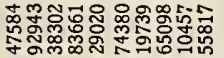

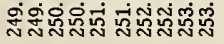

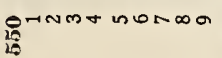

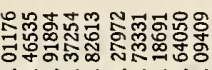

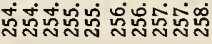
8-Nmtinoma : ง

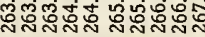
PrNmanona

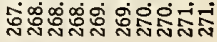
PrNm+unasa

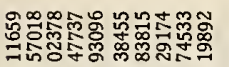

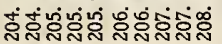

Brnmantma 군

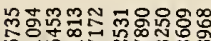

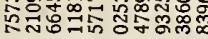

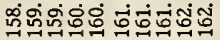

SHNm+ nONDa

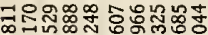

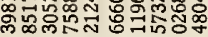

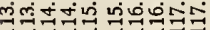

OrNm+ nURDa ลิ

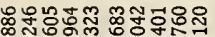

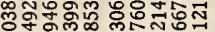

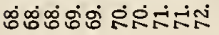
PrNmOnONA

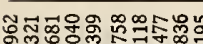

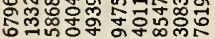

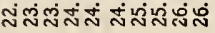
으

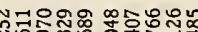

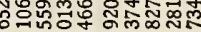

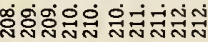
8

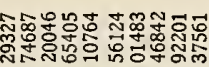

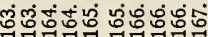

OndNm+ numba

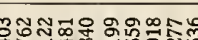

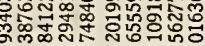

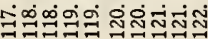
ค्र्थ

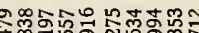

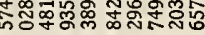

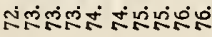

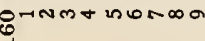

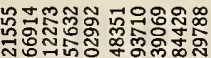

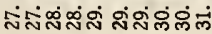
8

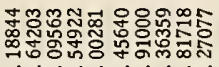

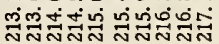

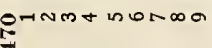

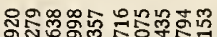

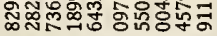

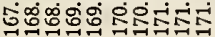

PNNM+nONa

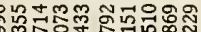

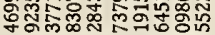

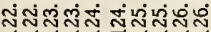

PrNm+nonos ต

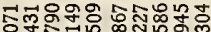

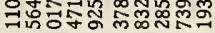

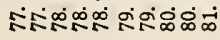
PrNm+nuNo

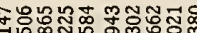

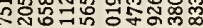

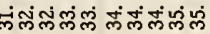

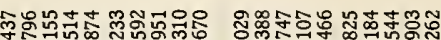
징융ำ

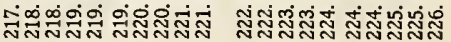

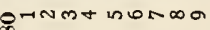
8

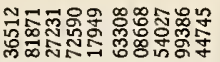

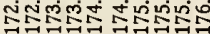
8

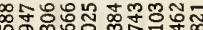

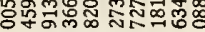

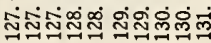

P्DNm+nONDO

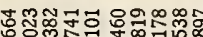

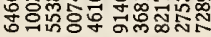

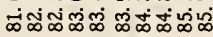

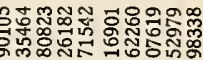

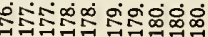

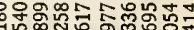

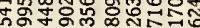

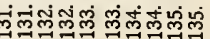

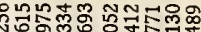
更

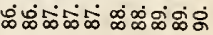

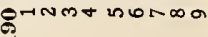

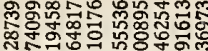

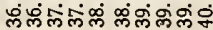
คrNmañona

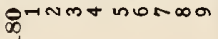

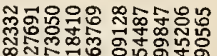

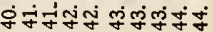 \&}




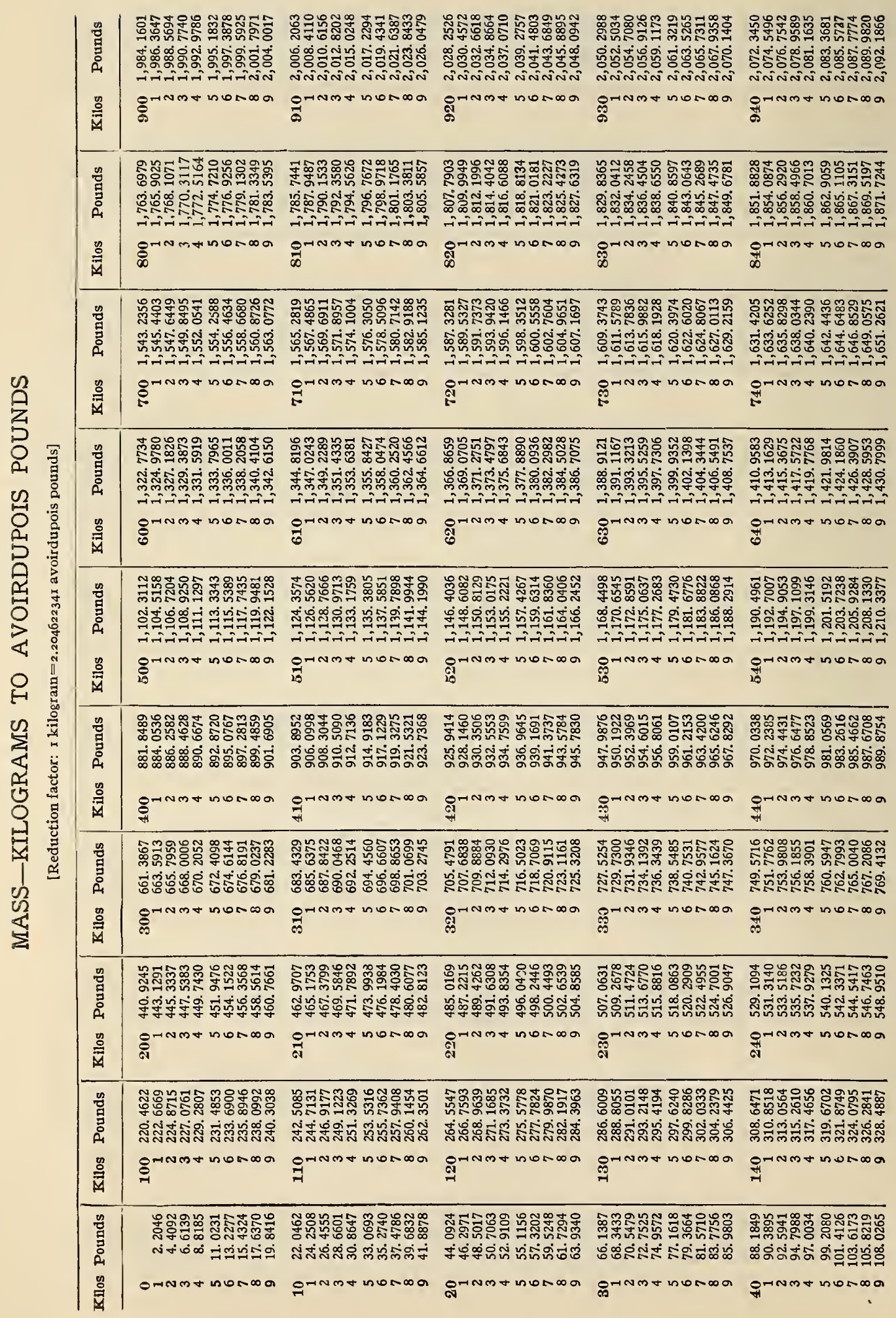




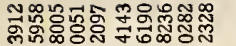

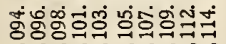

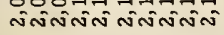

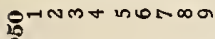
เू

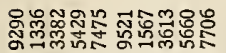
ஸ్య

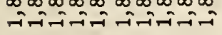
8

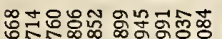

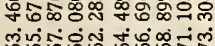

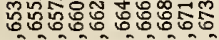

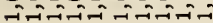

है

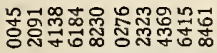

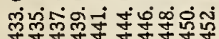

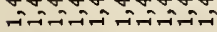

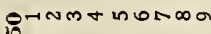

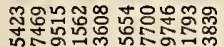

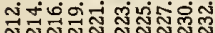

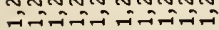
点

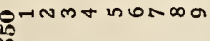

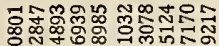

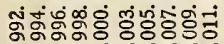
i-i-in-

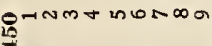

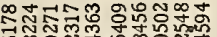

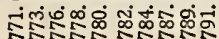

OHNmantina 0

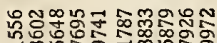
ที่

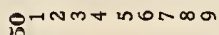
ผิ

tా

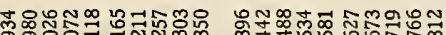

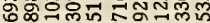

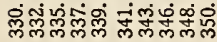
:

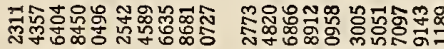

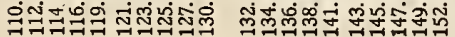

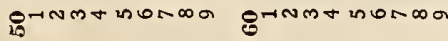

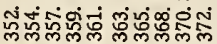
8rNmanuma

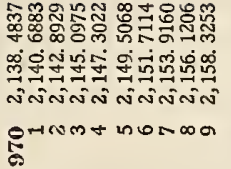

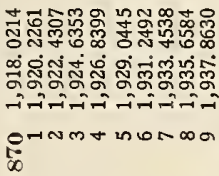

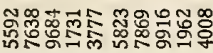

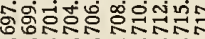

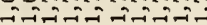

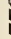

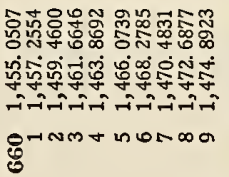

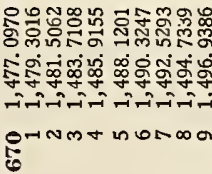

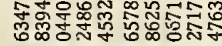
ஸ்-

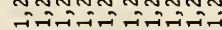$$
\text { 昆 }
$$

Prnmonorod

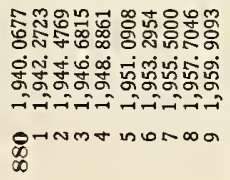

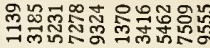

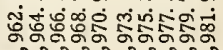
i-i-i-i $-1-i-1-1$ $\underset{\infty}{\infty}$

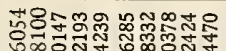

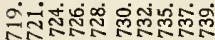

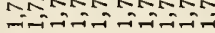
Ornmonono $\infty$ in

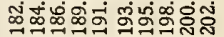

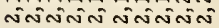
ळ

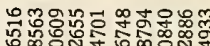

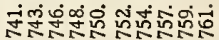

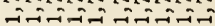

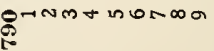

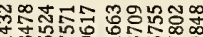

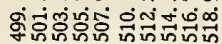

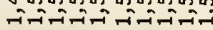

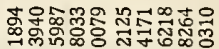

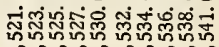

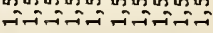
8

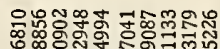

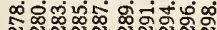

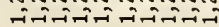
OrNmanorion ๑

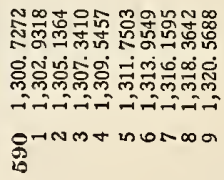

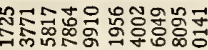

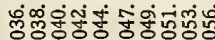
ininifinitiENm+unoma

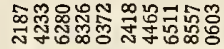

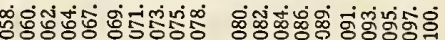

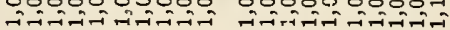

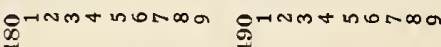

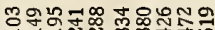

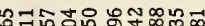

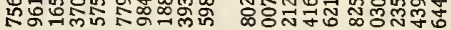

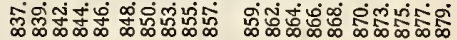

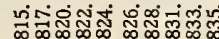

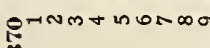
象 คำ

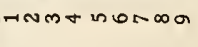

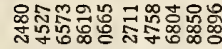

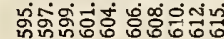
हैतnmanoma

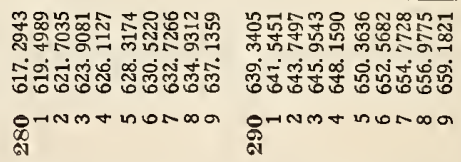

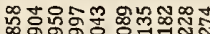

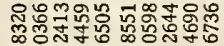

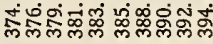

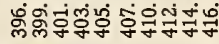

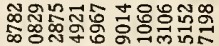
究

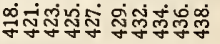
grnmonina

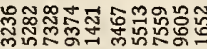

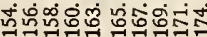

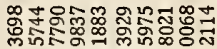

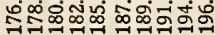

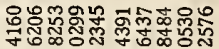

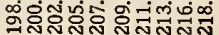
PrNmantman

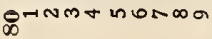
\&-NMTHUNDO 


\section{STATUS OF INTERNATIONAL METRIC SYSTEM IN THE UNITED STATES}

\subsection{CONSTITUTIONAL AUTHORITY TO FIX STANDARDS}

"The Congress shall have power to $* * *$ fix the standard of weights and measures." (Constitution of the United States, Art. 1, sec. 8, par. 5.)

\subsection{METRIC SYSTEM LEGALIZED}

"It shall be lawful throughout the United States of America to employ the weights and measures of the metric system, * * *." (Revised Statutes of the United States, sec. 3569.)

\subsection{METRIC STANDARDS ADOPTED AS FUNDAMENTAL}

"* * * the office of weights and measures $* * *$ will in the future regard the international prototype meter and kilogram as fundamental standards, and the customary units, the yard and the pound, will be derived therefrom in accordance with the act of July 28, 1866." (Order approved by the Secretary of the Treasury, April 5, 1893.)

\subsection{METRIC BALANCES FURNISHED CERTAIN POST OFFICES}

"The Postmaster General shall furnish to the post offices exchanging mails with foreign countries, and to such other offices as he may deem expedient, postal balances denominated in grams of the metric system, fifteen grams of which shall be the equivalent, for postal purposes, of one-half ounce avoirdupois, and so on in progression." (Revised Statutes of the United States, sec. 3880.)

\subsection{METRIC WEIGHTS USED FOR CERTAIN COINS}

“* * * the weight of the half-dollar shall be twelve grams and one-half of a gram; the quarter-dollar and the dime shall be, respectively, one-half and one-fifth of the weight of said half-dollar." (Revised Statutes of the United States, sec. 3513.)

10.6. CONGRESS PROVIDES METRIC STANDARDS FOR THE STATES OF THE UNION, LAND-GRANT AGRICULTURAL COLLEGES, AND THE SMITHSONIAN INSTITUTION

"Be it resolved by the Senate and House of Representatives of the United States of America in Congress assembled, That the Secretary of the Treasury be, and he is hereby, authorized and directed to furnish to each State, to be delivered to the governor thereof, one set of the standard weights and measures of the metric system for the use of the States, respectively." (Joint resolution of Congress approved July 27, 1866.)

"Resolved by the Senate and House of Representatives of the United States of America in Congress assembled, That the Secretary of the Treasury be, and he is hereby, directed to cause a complete set of all the weights and measures adopted as standards to be delivered to the governor of each State in the Union, for the use of agricultural colleges in the States, respectively, which have received a grant of lands from the United States, and also one set of the same for the use of the Smithsonian Institution: * * *." (Joint resolution of Congress, approved March 3, 1881.)

\subsection{UNITED STATES JOINS IN ESTABLISHING INTERNATIONAL BUREAU OF WEIGHTS AND MEASURES}

"The high contracting parties engage to establish and maintain, at their common expense, a scientific and permanent international bureau ${ }^{13}$ of weights and measures, the location of which shall be at Paris." (Extract from convention of 1875, signed for the United States by the United States ambassador to France.)

\footnotetext{
13 This bureau has the cutody of the international standards of the metric system, to which all metric prototypes of the world are referred for verification. It was estahlished and is maintained jointly by various governments.
} 


\subsection{CONGRESS ADOPTS THE METRIC UNITS FOR ELECTRICAL MEASURES}

It was enacted by the Congress in 1894 that the "international" electrical units should be the legal units of electrical measure in the United States. These units were based on the centimeter-gram-second electromagnetic system of electrical units, but for practical convenience were defined in terms of the electrical properties of silver and of mercury. In 1950 the Congress enacted Public Law 617-81st Congress (Chap. 484 2nd Session) which redefines the units directly in terms of the centimeter-gramsecond electromagnetic system of units and repeals the Act of 1894 (Public Law No. 105). Public Law 617 also defines the photometric units, candle and lumen, in terms of the light from one square centimeter of a perfect radiator at the temperature of freezing platinum.

\subsection{METRIC CARAT ADOPTED AS UNIT OF WEIGHT FOR DIAMONDS AND OTHER PRECIOUS STONES}

"Beginning July 1, 1913, the Bureau of Standards will recognize the international metric carat of 200 milligrams as the unit of weight for diamonds and other precious stones and will use this unit for the purposes of certification of all carat weights submitted to the bureau for test." (National Bureau of Standards, Circular C43, issued November 1, 1913.)

"On and after July 1, 1913, the unit of weight for imported diamonds, pearls, and other precious stones will be the metric carat of 200 milligrams.

"Collectors at ports now equipped with scales for weighing precious stones will make requisition for a new set of weights based upon such carat." (Treasury Department order, June 17, 1913, to collectors and other officers of the customs.)

\subsection{METRIC SYSTEM OPTIONAL IN PUERTO RICO}

"SECTION 1.-That the metric system of weights and measures and the imperial system customarily used in the United States, insofar as the same are described by this Act, are hereby recognized and established in Porto Rico for use in all industrial, commercial, or agricultural transactions, ***."

(Act No. 135, approved, August 18, 1913; last amended, 1936.)

\subsection{RELATION BETWEEN INCHES AND MILLIMETERS ADOPTED BY AMERICAN STANDARDS ASSOCIATION}

For industrial purposes a simple relation between the inch and the millimeter has been adopted by the American Standards Association (ASA B48.1-1933), and by similar organizations in 15 other countries. This relation is

$$
\begin{aligned}
1 \text { inch } & =25.4 \text { millimeters (exactly) } \\
\text { from which, } 1 \text { yard } & =0.9144 \text { meter (exactly). }
\end{aligned}
$$

The adoption of this relation by industry, for use in making conversions between inches and miilimeters, has no effect upon the legal definition of the yard or of the meter. 


\section{INDEX}

Abbreviations, list of accepted Acre:

Definition

Equivalents in hectares, 1 to 9 units

Equivalents in hectares, 1 to 999 unit

quivalents of bushels per acre in hectoliters per

hectare, 1 to 9 units.--

Relation to other area units.
merican Standards Association:

Conversion factor, inches to millimeters

Angstrom ...

Apothecaries units (see also under the nome of each unit)

Are, definition

Are, defir

Definition of units

Fundamental uni

Subsidiary units

A voirdupois units of mass (see also under the name of each unit)

Barrel:

For apples:

Definition

Volume in cubic inches

Board foot, definition

Bureau, International, of Weights and Measures. Bushel:

British:

Relation to United States bushel, approximate . -

United States:

Definition.

Equivalents in hectoliters, 1 to 9 units

Equivalents in hectoliters, 1 to 999 units

Heaped

Relation to other capacity units

Stricken or struck

Volume in cubic inches

Capacity:

Definition of units

Fundamental units

Subsidiary units

Capacity measures:

Coefficient of expansion

Distinction between, and units

Material of which made

Carat:

Metric, definition.

Old, in use in United States previous to July 1, 1913.

Centare, definition

Centigram, definition

Centiliter, definition

Centimeter:

Cubic:

Definition

Equivalents in cubic inches, 1 to 9 units

Relation to other volume units.................

Definition -

Relation to other units

Square:

Definition

Equivalents in square inches, 1 to 9 units

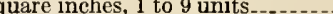

Chain (Gunter's):

Definition

Relation to other length units. Square:

Definition

Relation to other area units

Common fractions of an inch in decimals of an inch and

millimeters

Comparison of pounds and tons used in United States...-

Comparison of pounds and tons used in United States.---

Conversion factor, ASA:

Inches to millimeters

Cord, definition

unit)
Page

7 Customary standards of length, temperature at which

Decigram, definition

Deciliter, definition

Decimal and common fractions of an inch and milimeters. 8 Decimeter:

8 Cubic:

Relation to liter

Relation to other volume units..-

Definition

Equivalent in inches

Square, definition

Dekagram, definition

Dekaliter:

Definition

Equivalents in United States pecks, 1 to 9 units

Dekameter:

Cubic, definition.

Definition......-..-Dram:

Apothecaries:

Definition

Equivalents in

Relation to other units of mass-a.

Avoirdupois:

Definition

Relation to other units of mass

Fluid:

Definition

Equivalents in milliliters, 1 to 9 units Relation to other capacity units

Dry units of capacity (see also under the name of each unit)

Electrical units, based on metric system

Equivalents, fundamental Equivalents. (See names of units.)

Fathom, definition

Feet. (See Foot.)

Fluid units (see also under the name of each unit)

Foot:

Cubic:

Equivalents in cubic meters, 1 to 9 units

Equivalents in liters, 1 to 9 units..............

Relation to other volume units...................

\section{Definition}

Equivalents in meters, 1 to 9 units

Equivalents in meters, 1 to 999 units

(n)

Square:

Definition

Equivalents in square meters, 1 to 9 units....... Relation to other area units.

Fractions of an inch and millimeters...........................

Furlong, definition -

Gallon:

British imperial:

Relation to United States gallon, approximate...-

United States:

Definition

Equivalents in liters, 1 to 9 units

Equivalents in liters, 1 to 999 units..........

Relation to other capacity units

Volume in cubic inches

Geographical mile. (See Mile, geographical.)

15 Gill:

14

Definition.

Relation to other capacity units. Grain:

Volume in cubic inches

Definition

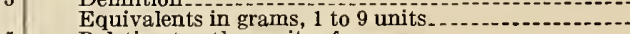

Relation to other units of mass
Page

5 
Gram:

Definition

Equivalents in grains, 1 to 9 units

Equivalents in apothecaries drams, 1 to 9 units Equivalents in troy ounces, 1 to 9 units............ Equivalents in avoirdupois ounces, 1 to 9 units...... Relation to other units of mass.

Weights of certain coins in grams.

Hand, definition

Hectare:

Definition

Equivalents in acres, 1 to 9 units

Equivalents in acres, 1 to 999 u nits....................... Relation to other area units

Hectogram, definition

Hectoliter:

Definition

Equivalents in United States bushels, 1 to 9 units Equivalents, 1 to 9 units:

Of hectoliters per hectare in United States hushel per acre

Equivalents of hectoliters per hectare in bushels per acre, 1 to 9 units

Hectometer:

Cubic, definition

Definition

Square, definition
Hundredths of an inch, equivalents of, in millimeters.....

Hundred weight

Long, definition

Short:

Definition

Relation to other units of mass

Inch:

Definition

Equivalents in millimeters, 1 to 9 units

Equivalcnts of common fractions of, in decimals of an inch

Equivalents of common fractions of, in millimeters.-. Equivalents in hundredths of an inch, in millimeters Equivalents in meters, 1 to 12 units

Relation to other length units

Cuhic:

Definition

Equivalents in cubic centimeters, 1 to 9 units.-.-

Equivalents in liters, 1 to 9 units.

Number in gallon.

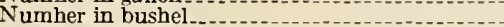

Relation to dry capacity units

Relation to liquid capacity units

Square:

Definition

Equivalents in square centimeters, 1 to 9 units.-.

Relation to ot her area units

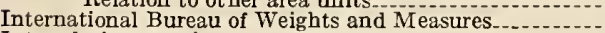

Interrelation of units.

Introduction.

Kilogram:

Definition

Equivalents in avoirdupois pounds, 1 to 9 units......

Equivalents in avoirdupois pounds, 1 to 999 units

Equivalents in avoirdupois and troy pounds, short

and long tons, and metric tons, 1 to 9 units.........

Relation to other units of mass........-

Kilometer:

Cuhic, definition

Definition.

Equivalents in statute miles, 1 to 9 units

Equivalents in statute miles, 1 to 999 units ...........

Square:

Definition

Equivalents in square miles, 1 to 9 units

Law:

Equivalent of half ounce in grams for use in post offices

Electrical units based on metric system

July 28,1866 , legalizing use of metric system

Metric system in Puerto Rico

Weights of certain coins in grams fixed by law ----

Legal status of metric system in United States........

Length:

Cahle. (See Cable length.)

Definition of units

Fundamental units
Page

Link:

Definition

Square:

Definition

Relation to other area units

Liquid units (see also under the name of each unit) .......Liquids:

Sale of, by measure

Liter:

:

Equivalents in cubic inches, 1 to 9 units

In cubic feet, 1 to 9 units...........................

In United States dry quarts, 1 to 9 units

In United States dry pecks, 1 to 9 units

In United States liquid quarts, 1 to 9 units.......-

In United States gallons, 1 to 9 units

In United States liquid quarts, 1 to 999 units.-.-.

In United States gallons, 1 to 999 units

Relation to cubic decimeter

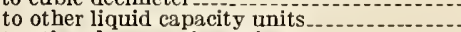
to other dry capacity units

3 Long hundred weight. (See Hundredweight, long.)

Long ton. (See Ton, long.)

Mass:

Definition of units

Fundamental units

Suhsidiary units

6 Mendenhall Order.

Meter:

6 Cubic:

Definition

Equivalents in cuhic feet, 1 to 9 units
Equivalents in cuhic yards, 1 to 9 units

Equivalents in cuhic yards, 1 to 999 units

Refinition

Equivalents in feet, 1 to 9 units

Equivalents in feet, 1 to 999 units

Equivalents in rods, 1 to 9

Equivalents in yards, 1 to 9 units

Legal equivalent in inches.

Relation to other length units

Square:

Definition

Equivalents in square feet, 1 to 9 units

Equivalents in square yards, 1 to 9 units..........

Relation to other area units 1 to

Metric standards of length, temperature at which standard

Metric system:

A hbreviations

Legalized.

of legal status

Units

Use in coinage

Use in Puerto Rico

Metric ton. (See Ton, metric.)

Mile:

Geographical, definition

Nautical:

Definition

Square:

Definition

Equivalents in square kilometers, 1 to 9 units
Relation to other area units.-. Statute:

Definition

Equivalents in kilometers, 1 to 9 units

Equivalents in kilometers, 1 to 999 units

Relation to other length units.................-.

Milligram:

Definition

Relation to other units of mass............................

Milliliter:

Definition

Equivalent in cubic centimeters

60 Equivalent in cubic centimeters $\quad$ Equivalents in apothecaries drams, 1 to 9 units.......

Equiralents in fluid ounces, 1 to 9 units..............

Relation to other capacity units.

Millimeter:

Cuhic, definition

Equivalents in

Equivalents in inches, 1 to 9 units

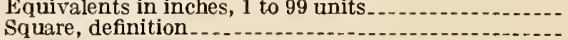

Square, definition
Millimicron, definition
Page 
Minim:

Definition

Relation to other capacity units

Nautical mile. (See Mile, nautical.)

Ounce:

A pothecaries definition

Avoirdupois:

Definition

Equivalents in grams, 1 to 9 units

Fluid:

Definition

Equivalents in milliliters, 1 to 9 units

Troy:

Relation to other capacity units

Definition

Equivalents in grams, 1 to 9 units

Relation to other un

Peck:

Definition

Equivalents in liters, 1 to 9 units

Relation to other capacity units

Volume in cubic inches

Pennyweight:

Relation to otber units of mass Pint:

Dry:

Definition

Relation to other capacity units

iquid:

Definition

Equivalents in liters, 1 to 9 units

Relation to other capacity units

Volume in cubic inches

Point, definition.

Post office, equivalent of balf ounce in usage Pound:

Apothecaries:

Definition

Relation to otber units of mass

Avoirdupois:

Definition

Equivalents in kilograms, 1 to 9 units

Equivalents in kilograms, 1 to 999 units

Equivalents in troy pounds, kilograms, sbort and long tons, and metric tons, 1 to 9 units

Fundamental equivalent .............................

Relation to other units of mass.

Troy:

Definition Equivalents in avoirdupois pounds, kilograms,
short and long tons, and metric tons, 1 to 9 units.

Relation to other units of mass

Quart:

Dry:

Definition

Equivalents in liters, 1 to 9 units.

Relation to other capacity units

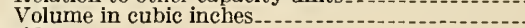

Liquid:

Definition.

Equivalents in liters, 1 to 9 units

quivalents in liters, 1 to 990 units

Relation to other capacity units

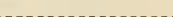

Volume in cubic inches

Reduction factors (see also separate tables).

Rod:

Definition

Equivalents in meters, 1 to 9 units

Relation to

Definition

Relation to other area units
Page

Scruple, apothecaries:

Definition

Relation to other units of mass

Short humdredureight. (See Hundredr

edweight, short.)

Short ton. (See Ton, short.)

Span, definition.

Sp

Square measure:

Abbre

See also under the name of each unit

Standards, metric, furnished to each State

Standards of measurement:

Coefficient of

Congress has power to fix, in Constitution.

Density of, for weights

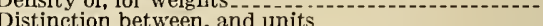

Temperature of

Status of metric system in United States-a.

Statute mile. (See Mile, statute.)

Tol

\section{Weights:} Buoyancy-1.

Material

Weights and Measures, International Bureau............

Yard:

Cubie:

Definition

Equivalents in cubic meters, 1 to 9 units

Equivalents in cubic meters, 1 to 999 units

Equivalents in cubic meters, 1 to 999 units

Relation to other volume units

Equivalents in meters, 1 to 9 units

Legal equivalents

Relation to other length units.

Square:

Definition

Equivalents in square meters, 1 to 9 units
Relation to other area units.
Page 

